\title{
Comprehensive Control of Human Papillomavirus Infections and Related Diseases
}

\author{
F. Xavier Bosch ${ }^{\mathrm{a},{ }^{*}}$, Thomas R. Broker ${ }^{\mathrm{b}}$, David Forman ${ }^{\mathrm{c}}$, Anna-Barbara Moscicki ${ }^{\mathrm{d}}$, Maura L. \\ Gillison $^{\mathrm{e}}$, John Doorbar ${ }^{\mathrm{f}}$, Peter L. Stern ${ }^{\mathrm{g}}$, Margaret Stanley ${ }^{\mathrm{h}}$, Marc Arbyn ${ }^{\mathrm{i}, \mathrm{j}}$, Mario Poljak ${ }^{\mathrm{k}}$, \\ Jack Cuzick', Philip E. Castle ${ }^{m}$, John T. Schiller ${ }^{n}$, Lauri E. Markowitz ${ }^{\circ}$, William A. Fisher ${ }^{p}$, \\ Karen Canfellq, Lynette A. Denny ${ }^{r}$, Eduardo L. Francos, Marc Steben ${ }^{t}$, Mark A. Kane ${ }^{u}$, Mark \\ Schiffman $^{\mathrm{v}}$, Chris J.L.M. Meijer ${ }^{\mathrm{w}}$, Rengaswamy Sankaranarayanan ${ }^{\mathrm{x}}$, Xavier Castellsaguéa, ${ }^{a}$, \\ Jane J. Kim ${ }^{z}$, Maria Brotons ${ }^{a}$, Laia Alemany ${ }^{a}, y$, Ginesa Albero ${ }^{a}, y$, Mireia Diaz ${ }^{a}$, and Silvia de \\ Sanjoséa,y on behalf of the authors of the ICO Monograph 'Comprehensive Control of HPV \\ Infections and Related Diseases' Vaccine Volume 30, Supplement 5, 2012
}

aCancer Epidemiology Research Program (CERP), Institut Català d'Oncologia - Catalan Institute of Oncology (ICO), IDIBELL, L'Hospitalet de Llobregat (Barcelona), Spain bUniversity of Alabama at Birmingham, Biochemistry and Molecular Genetics, Birmingham, Alabama, USA 'Section of Cancer Information, International Agency for Research on Cancer, Lyon, France dDivision of Adolescent Medicine, Department of Pediatrics, University of California, San Francisco, San Francisco, CA, USA eViral Oncology, The Ohio State University Comprehensive Cancer Center, Columbus, OH, USA fDivision of Virology, National Institute for Medical Research, London, UK gPaterson Institute for Cancer Research, University of Manchester, Manchester, UK hDepartment of Pathology, Tennis Court Road, Cambridge, UK iUnit of Cancer Epidemiology, Scientific Institute of Public Health, Brussels, Belgium 'Laboratory for Cell Biology and Histology, University of Antwerp, Antwerp, Belgium kInstitute of Microbiology and Immunology, Faculty of Medicine, University of Ljubljana, Ljubljana, Slovenia 'Wolfson Institute of Preventive Medicine, Queen Mary University of London, London, UK ${ }^{m}$ Global Cancer Initiative, Chestertown, MD, USA ${ }^{\mathrm{n} C e n t e r}$ for Cancer Research, National Cancer Institute, Bethesda, MD, USA ${ }^{\circ}$ National Center for HIV, Viral Hepatitis, STD and TB Prevention, Centers for Disease Control and Prevention, Atlanta, GA, USA PDepartment of Psychology and Department of Obstetrics and Gynaecology, University of Western Ontario, Social Sciences Centre 7428, London, Ontario, Canada 9Lowy Cancer Research Centre, Prince of Wales Clinical School, The University of NSW, Australia and Cancer Epidemiology Research Unit, Cancer Council NSW, Sydney, Australia (past affiliation) 'Department Obstetrics and Gynaecology and Institute of Infectious Diseases and Molecular Medicine, University of Cape Town/Groote Schuur Hospital, Cape Town, South Africa sDivision of Cancer Epidemiology, McGill University, Montreal, Canada tInstitut National de Santé Publique du Québec, Montréal, Québec, Canada "Consultant on Immunization Policy, Mercer Island, WA, USA vational Cancer Institute, Division of Cancer Epidemiology and Genetics, Bethesda, MD, USA wDepartment of Pathology, VU University Medical Centre, Amsterdam, The Netherlands xScreening Group, International Agency for Research on Cancer, Lyon, France yCIBER en Epidemiología y Salud Pública (CIBERESP), Spain ${ }^{\mathrm{z} C e n t e r ~ f o r ~ H e a l t h ~ D e c i s i o n ~ S c i e n c e, ~}$ Department of Health Policy and Management, Harvard School of Public Health, Boston, MA, USA 


\section{Abstract}

(C) 2013 Elsevier Ltd. All rights reserved.

*Corresponding author. Tel.: +34 93 2607812; fax: +34 93 2607787. admincerp@ iconcologia.net (F.X. Bosch).

Disclosed potential conflicts of interest

FXB: Has received occasional travel grants to conferences/symposia/meetings and honorarium by either GlaxoSmithKline, Merck, Sanofi Pasteur MSD, Roche or Qiagen. The Unit of Infections and Cancer at the ICO is involved in HPV vaccine trials and epidemiological studies sponsored by GlaxoSmithKline, Merck and Sanofi Pasteur MSD.

TRB: Has disclosed no potential conflicts of interest.

DF: Has disclosed no potential conflicts of interest.

ABM: serves as an advisory board member for Merck and receives support for sexually transmitted disease testing from Gen-Probe. She has also received travel funds and an honorarium for speaking at a symposium supported by Becton Dickinson.

MLG: Has had scientific collaborations and has received research funding from Merck. She has acted as a consultant for Merck and GlaxoSmithKline.

JD: Is supported by the UK Medical Research Council, has recently acted as consultant for Sanofi Pasteur MSD, Merck and Roche, and has received research support from Sanofi Pasteur MSD, GlaxoSmithKline and the Wellcome Trust.

PLS: Has received support for Travel, Lectureships, (GlaxoSmithKline); Consultancy (GlaxoSmithKline, Oxford Biomedica); Meeting/Travel expenses (GlaxoSmithKline, Oxford Biomedica).

M. Stanley: Has acted as a consultant for MSD Merck, Sanofi Pasteur MSD and GlaxoSmithKline.

MA: Has disclosed no potential conflicts of interest.

MP: Advisory Board (Roche); Consultant (Abbott); Research Grants (Abbott, Roche); Speakers Bureau (Abbott, Roche); Travel Grants (Abbott, Roche).

JC: Is on advisory Boards for Abbott, Becton Dickinson, Gen-Probe, Qiagen and Roche and his institution receives research funding from these companies as well as Genera Biosystems and Oncohealth.

PEC: Serves as a member of a Data and Safety Monitoring Board to review data on HPV vaccines for Merck, has received HPV test reagents and testing for research from Qiagen and Roche at a reduced or no cost, and has consulted for BD, Gen-Probe/Hologic, Roche, Cepheid, and GE Healthcare.

JTS: Named inventor on U.S. government-owned HPV vaccine-related patents that are licensed to Merck \& Co., GlaxoSmithKline, Sanofi Pasteur and Shantha Biotechnics and is entitled to limited royalties as specified by federal law.

LEM: Has disclosed no potential conflicts of interest.

WAF: Has received speaker fees, and educational and unrestricted research grants from Merck Canada.

KC: Is co-PI of a new trial of primary HPV screening in Australia, which is partially supported by Roche Molecular Diagnostics USA. LAD: Has received honoraria from GlaxoSmithKline and Merck for appearing on various speaker fora and sponsorship for research studies.

ELF: Has served as occasional consultant to companies involved with HPV vaccines (Merck and GlaxoSmithKline) and with HPV diagnostics (Roche, Gen-Probe, Becton Dickinson). He has received an unrestricted grant from Merck.

M. Steben: Consultant (Roche Molecular, Digene and Graceway Pharma); Research Grants (Merck and GlaxoSmithKline); Speakers Bureaus (Merck, Graceway Pharma, Digene and Laboratoire Biron); Educational presentations (Merck and Graceway Pharma); Payment for manuscript preparation (Graceway Pharma).

MAK: Member of the Merck Advisory Board for HPV vaccine and has received consulting fees, honoraria and travel support from Merck.

M. Schiffman: Has received free CareHPV equipment and reagents for an independent analysis of low-cost HPV testing in Africa. He has also received free specimen testing from Roche for epidemiologic projects. He was the NCI co-Project Officer and co-Medical Monitor of an independent evaluation of GlaxoSmithKline HPV vaccine, for which GlaxoSmithKline donated vaccine and financed the regulatory components.

CJLMM: Scientific advisory board (Qiagen); Speakers office (GlaxoSmithKline, Roche); Research Grant (Abbott); Shares minority (Self-Screen B.V).

RS: Has disclosed no potential conflicts of interest.

XC: Has received occasional travel grants to scientific meetings and honorarium for consultancy by either GlaxoSmithKline, Merck, Sanofi Pasteur MSD. The Unit of Infections and Cancer at the ICO is involved in HPV vaccine trials and epidemiological studies sponsored by GlaxoSmithKline, Merck and Sanofi Pasteur MSD.

JJK: Has disclosed no potential conflicts of interest.

MB: Has received occasional travel grants to conferences by GlaxoSmithKline or Sanofi Pasteur MSD. The Unit of Infections and Cancer at the ICO is involved in HPV vaccine trials and epidemiological studies sponsored by GlaxoSmithKline, Merck and Sanofi Pasteur MSD.

LA: Has received occasional travel grants to conferences by Merck and Sanofi Pasteur MSD. The Unit of Infections and Cancer at the ICO is involved in HPV vaccine trials and epidemiological studies sponsored by GlaxoSmithKline, Merck and Sanofi Pasteur MSD.

GA: Has received occasional travel grants to conferences/meetings by either GlaxoSmithKline, Merck, Sanofi Pasteur MSD, Roche or Qiagen. The Unit of Infections and Cancer at the ICO is involved in HPV vaccine trials and epidemiological studies sponsored by GlaxoSmithKline, Merck and Sanofi Pasteur MSD.

MD: Has disclosed no potential conflicts of interest. The Unit of Infections and Cancer at the ICO is involved in HPV vaccine trials and epidemiological studies sponsored by GlaxoSmithKline, Merck and Sanofi Pasteur MSD.

SdS: Has received occasional travel grants to conferences/symposia/meetings by either GlaxoSmithKline, Sanofi Pasteur MSD or Qiagen. The Unit of Infections and Cancer at the ICO is involved in HPV vaccine trials and epidemiological studies sponsored by GlaxoSmithKline, Merck and Sanofi Pasteur MSD. 
Infection with human papillomavirus (HPV) is recognized as one of the major causes of infectionrelated cancer worldwide, as well as the causal factor in other diseases. Strong evidence for a causal etiology with HPV has been stated by the International Agency for Research on Cancer for cancers of the cervix uteri, penis, vulva, vagina, anus and oropharynx (including base of the tongue and tonsils). Of the estimated 12.7 million new cancers occurring in 2008 worldwide, $4.8 \%$ were attributable to HPV infection, with substantially higher incidence and mortality rates seen in developing versus developed countries. In recent years, we have gained tremendous knowledge about HPVs and their interactions with host cells, tissues and the immune system; have validated and implemented strategies for safe and efficacious prophylactic vaccination against HPV infections; have developed increasingly sensitive and specific molecular diagnostic tools for HPV detection for use in cervical cancer screening; and have substantially increased global awareness of HPV and its many associated diseases in women, men, and children. While these achievements exemplify the success of biomedical research in generating important public health interventions, they also generate new and daunting challenges: costs of HPV prevention and medical care, the implementation of what is technically possible, socio-political resistance to prevention opportunities, and the very wide ranges of national economic capabilities and health care systems. Gains and challenges faced in the quest for comprehensive control of HPV infection and HPVrelated cancers and other disease are summarized in this review. The information presented may be viewed in terms of a reframed paradigm of prevention of cervical cancer and other HPV-related diseases that will include strategic combinations of at least four major components: 1) routine introduction of HPV vaccines to women in all countries, 2) extension and simplification of existing screening programs using HPV-based technology, 3) extension of adapted screening programs to developing populations, and 4) consideration of the broader spectrum of cancers and other diseases preventable by HPV vaccination in women, as well as in men. Despite the huge advances already achieved, there must be ongoing efforts including international advocacy to achieve widespread — optimally universal-implementation of HPV prevention strategies in both developed and developing countries.

This article summarizes information from the chapters presented in a special ICO Monograph 'Comprehensive Control of HPV Infections and Related Diseases' Vaccine Volume 30, Supplement 5, 2012. Additional details on each subtopic and full information regarding the supporting literature references may be found in the original chapters.

\section{Keywords}

HPV; Cervical cancer; Anal cancer; Penile cancer; Vaginal cancer; Vulvar cancer; Oropharyngeal cancer; Screening; HPV vaccination; HPV testing; Prevention

\section{Global prevention and management of HPV related diseases: the pressing challenges and the compelling opportunities [1]}

\subsection{Introduction}

The scientific community has gained tremendous knowledge about human papillomaviruses (HPVs) and their interactions with host cells, tissues and immune systems; has validated and implemented strategies for prophylactic vaccination against HPV infections; has developed 
increasingly sensitive and specific molecular diagnostic tools; and has substantially increased global awareness of HPV and the many associated diseases of women, men, and children. In so doing, we have come up against new and daunting challenges: costs of HPV prevention and medical care, the implementation of what is technically possible, the diverse societal standards around the globe concerning reproductive health, and the very wide ranges of national economic capabilities and health care systems. HPV is one of the few agents causing disease and cancer where the emerging opportunities for prevention have encountered some socio-political resistance, the nature of which depends on country and culture. In addition, there has been a resistance for policy makers, funding agencies and corporate stakeholders to discount the need for significant new developments, particularly in the arenas of therapeutics and affordable interventions. An expanded repertoire of health care options is urgently needed to bring HPV under short-term management and long-term elimination. Thus, we are at crossroads that will require thoughtful discussions, compassionate decisions and concerted actions.

\subsection{Appreciation of disease causality and need for management}

There is inadequate recognition of the social impact of the successive stages of HPV infections: the infants who acquire low-risk HPV types 6 and 11 and develop recurrent respiratory papillomatosis (RRP); the adolescents with benign but highly contagious infections; the middle aged with consequences on reproductive capabilities and well being of the mother; the older persons with an increasing risk of cancer. Of the major cancers of women, cervical cancer tragically results in the most years of life expectancy lost (estimated at 29 years), considerably more than for women succumbing to breast cancer. This places an exceptional—and avoidable — burden on young families, and to a very serious degree on children who lose their mother. Yet these facts have not moved sufficiently into the public discourse. There is also a troubling disjunction between the popular culture, celebrities, and the media, entertainment and fashion industries, which popularize sexuality and beauty versus the need for candid and frank discussion about sexual health and the impact of sexually transmitted infections. The biological reality is that early age exposure to and frequent reinfection with mucosotropic HPV types can have serious long-term outcomes in the form of future cervical, penile, vulvar, vaginal and anal cancer, RRP and certain head and neck afflictions.

\subsection{The success and promise of prophylactic vaccines}

The quadrivalent and bivalent HPV vaccines have proven to be very safe, with long-term durability of protection against primary infection with vaccine types and a moderate degree of cross-protection against some non-vaccine types. Next-generation vaccines targeting additional oncogenic genotypes are completing clinical trials.

Childhood vaccination programs are nearly universal. However, there are few effective strategies for vaccinating pre-adolescents, the currently recommended age for HPV vaccination. A good case can be made for clinical trials to evaluate vaccination at younger ages, within the schedules of pediatric vaccination against other common viral infections to improve coverage and to eliminate the negative rhetoric about the vaccines possibly altering sexual behavior. The significantly stronger immune response to the HPV vaccines at 
younger ages, compared to adolescents, combined with the durability of protection from infection that is becoming demonstrable, and the small but real risk of HPV infection of children as a result of abuse, together suggest that early vaccination of toddlers could become good public health policy.

\subsection{A range of diagnostic methods}

Diagnostic screening for HPV lesions is generally available in the developed world but scarce everywhere else for lack of public health policy, professional and general education, media awareness, clinical settings, financial resources and, most crucially, insufficient capacity for effective follow-up treatment of identified lesions. Screening is typically visual and subjective, with even the most highly trained experts sometimes are unable to agree. New tests for molecular biomarkers of infection and disease have greatly improved sensitivity, reliability and are beginning to help define the degree of present and future risk.

\subsection{The potential for therapeutic inhibition of persistent infections}

As the essential follow-up to disease diagnosis, new therapies are needed and they must be effective, minimally impactful on normal tissues and organs, affordable, and available everywhere. The armamentarium of therapeutic capabilities will necessarily include therapeutic vaccines and specific immune stimulants to augment natural immunity. Molecular-based therapies are absolutely essential partners in disease prevention and management.

Arguably, screening, early detection and (when eventually possible) relatively simple therapeutic intervention could emerge as more practical than attempts at annual global vaccination of some 100 million children (of any given age cohort), unless and until vaccination becomes affordable, deliverable, and essentially universal and has substantially broader coverage to include most pathogenic mucosotropic genotypes.

\subsection{The time for universal response is now}

HPV is an equal opportunity pathogen. It is part of the human condition, well adapted to infecting the epithelia and so prevalent as to be almost unavoidable. Unlike the acute viruses that establish severe diseases shortly after infection, the modus operandi of HPV infections is primarily latent, subclinical, and opportunistic for sporadic reproduction and transmission, and HPV is generally in a state of quasi-equilibrium with the host. HPV is a real agent of affliction, a social disease of a sociable human species that is highly communicable, and susceptibility to it is definitely not a lifestyle choice.

Societies need to acknowledge the extraordinary prevalence and virtual unavoidability of infection and put aside any inclination to cast blame, exploit shame, or castigate people for acquisition of HPV infections. Their importance as a precursor to carcinomas should not be dismissed for any reason. In fact, these diseases are the leading preventable cancers against which humanity can readily make progress at reduction. 


\section{Reframing cervical cancer prevention. Expanding the field towards prevention of HPV infections and related diseases [2]}

\subsection{HPV and cervical cancer: natural history and prevention opportunities}

Cervical cancer is the rare end stage of an unresolved HPV infection, currently defined as a persistent presence of the HPV DNA in repeated testing of cervical specimens. In many industrialised countries the prevalence of HPV infections in young adult females is as high as $40-80 \%$ and the lifetime probability of ever encountering HPV is as high as $80-90 \%$. Most of these infections clear spontaneously without clinical signs or symptoms. The fraction of persistent carriers of HPV in the middle ages is estimated in a range of 4-10\% and these women are the true high-risk group for cervical cancer and probably for any other HPV related cancer. The underlying endogenous and exogenous factors driving the process to clearance or persistent infection are still unclear. The time lag between the peak of HPV infection and the peak of cancer incidence is two to four decades, making the initiating infections and precursor lesions of cervical cancer an appropriate target for screening and early detection. Despite significant gaps in knowledge, cervical cancer is the best understood of all cancers and model system for carcinogenesis.

\subsection{HPV and other cancers and conditions: expanding the spectrum of disease prevention}

In cancers of the vagina, and their precursor lesions, HPV DNA is detected in the majority of cases. In recent reviews, between $64 \%$ and $91 \%$ of vaginal cancer cases and $82 \%$ and $100 \%$ of vaginal intraepithelial neoplasia grade 3 lesions were HPV DNA-positive. An estimated $40-50 \%$ of cancers of the vulva have also been associated with HPV. In men, HPV DNA is regularly found in cancers of the penis (40-50\%). In both sexes, HPV DNA is detected in anal cancers (88-94\%). In head and neck cancers, the prevalence of HPV DNA varies greatly by study, cancer site and geography. The most consistent finding relates to the oropharyngeal cancers, where HPV DNA has been found in 35-50\% in developed countries in contrast with the rest of the oral cavity, where HPV DNA is found in 5-15\% of the cases. In all HPV-positive noncervical cancers, HPV16 is the most common HPV type detected, followed by HPV types like18, 31, 33 and 45. Among the non-cancerous HPV-associated preventable conditions, genital warts (GW) and RRP are unequivocally linked to HPV6 and 11.

Current vaccines have been shown to protect against type-specific preneoplastic lesions in the genital tract of females and males (cervix, vulva, vagina and anus) and against GW in both sexes, thus expanding the potential for prevention.

Fig. 1 shows schematically the natural history of HPV infections on an age scale and the foreseeable strategic combinations for cervical cancer prevention.

Strategy 1 shows scenarios in which conventional screening exists and is firmly established. These are applicable to primarily industrialised countries and their programs represent transitional stages until HPV vaccination is generalized. Screening can remain based on the Pap smear, can employ novel forms of automated reading of cytology or can use HPV nucleic acid detection technology (DNA and RNA tests) with or without novel biomarkers. 
Second generation screening alternatives (HPV-based) have shown significant increases in sensitivity as compared to cytology with a moderate loss of specificity, allowing for increased screening intervals, a reduction of the total number of lifetime screening events without compromising safety, and a reduced total number of invasive cervical cancers detected.

Strategy 2 is likely to represent many developing countries and emerging economies in which routine screening has been shown to be unsustainable in the past and where routine immunization appears as the primary component of the preventive strategy. Screening in these circumstances might develop slowly and few lifetime screening events (2 to 4 ) may be offered under population programs. Novel low cost HPV technologies paired with screenand-treat protocols might be the model for large populations in the world.

Strategy 3 is likely to be adopted by most industrialised countries and some low- and middle-income countries in which combinations of generalized immunization of adolescents and HPV screening of adult women will coexist until broad spectrum vaccines become established.

\subsection{Elements of paradigm change in HPV-related cancer prevention}

Table 1 summarizes the elements in research that are prompting a paradigm change for prevention of HPV infection and related disease.

The first item refers to the recognition of the scope of diseases that are now firmly linked to persistent HPV infections, namely the canopy of cancers related to HPV in both genders. Second is the recognition that HPV vaccines are equally effective in men and women and third is the increasing accessibility to HPV vaccines of all populations worldwide. Item four in Table 1 calls for the need to conduct additional clinical trials that would facilitate integration of the HPV vaccines into the routine Expanded Program on Immunization (EPI) vaccination platform.

The reframed paradigm of cervical cancer prevention will thus include strategic combinations of at least four major components:

1. Routine introduction of HPV vaccines to women in all countries and in the widest possible age ranges.

2. Extension of coverage and simplification of the algorithms of existing screening programs using HPV based technology.

3. Extension of adapted screening programs to adult women in developing populations.

4. Consideration of the broader spectrum of cancers and other diseases preventable by HPV vaccination in women and evaluation of the importance of HPV vaccination in men. 


\section{Global burden of HPV and related diseases [3]}

\subsection{HPV prevalence}

The global prevalence of HPV infection in women with normal cytology is around 11-12\%, with the highest prevalences in sub-Saharan Africa (24\%), Eastern Europe (21\%) and Latin America (16\%) (Fig. 2). Maximum rates of HPV prevalence are observed in women less than 25 years, declining in older ages in many populations, some of which have a secondary peak in peri-menopausal or early menopausal women. In other populations, like in China, the prevalence of HPV is relatively age independent. The explanation of these difference prevalence patterns and the clinical significance is not understood. The five most prevalent types worldwide are HPV16 (3.2\%), HPV18 (1.4\%), HPV52 (0.9\%), HPV31 (0.8\%) and HPV58 $(0.7 \%)$, although these estimates represent point prevalence and not cumulative exposure and will, therefore, be underestimates.

Prevalence increases in women with cytologic cervical pathology in direct proportion to the severity of the lesion, reaching around $90 \%$ in women with cervical intraepithelial neoplasia (CIN) grade 3 and invasive cervical cancer. Careful retrospective investigations have shown that nearly $100 \%$ of all cervical cancer is HPV positive. Notably, the proportion of HPVpositive women in whom HPV16 is detected greatly increases with lesion severity (Table 2).

\subsection{Cancers attributable to HPV infection: global and regional burden of cervical cancer in 2008}

Infection with high-risk HPV types is recognized as one of the major causes of infectionrelated cancer worldwide. Strong evidence for a causal etiology with HPV has been stated by the International Agency for Research on Cancer for cancers of the cervix uteri, penis, vulva, vagina, anus and oropharynx (including base of the tongue and tonsils).

Table 3 shows the estimated number of cancers attributed to HPV classified by geographic region. Of the estimated 12.7 million new cancers occurring in 2008 worldwide, 700,000 occurred at an HPV-associated cancer site and 610,000 of these were attributable to HPV. This represents $4.8 \%$ of the total burden of cancer worldwide (Population Attributable Fraction $[\mathrm{PAF}])$. This PAF varies widely by geographic region, ranging from $1.2 \%$ in Australia and New Zealand to $14.2 \%$ in sub-Saharan Africa and $15.5 \%$ in India. Of note, $80.6 \%$ (490,000 cases) of the total number of cases attributable to HPV occurred in less developed regions (6.9\% of the global cancer burden in these regions) compared with 120,000 in more developed regions ( $2.1 \%$ of the global cancer burden in such regions).

Cervical cancer, for which PAF is assumed to be $100 \%$, is the third most common female cancer worldwide, with an estimated 530,000 new cases in 2008 (approximately 86\% of these cases occurred in less developed regions). The other five HPV-related cancer types accounted for the residual 80,000 cancer cases (see Table 3).

There is a well-characterized and strong association between cervical cancer incidence and level of development. Incidence and mortality rates tend to be at least four-fold higher in low Human Development Index (HDI) countries compared with very high HDI countries. A similar pattern is observed for 5-year relative survival, with less than $20 \%$ in low HDI 
countries and more than $65 \%$ in very high HDI countries. Global maps of cervical cancer rates show patterns of variation largely consistent with level of HDI (Fig. 3).

\subsection{Temporal trends in cancers attributable to HPV}

In countries for which reliable temporal data are available, incidence rates appear to be consistently declining by approximately $2 \%$ per annum. The decline is predominantly a result of effective population-based screening procedures, combined with sociocultural factors. There is however, a lack of information from low HDI countries where screening is less likely to be successfully implemented and cervical cancer often represents the most frequent diagnosed cancer type (Fig. 4). Projected global estimates of invasive cervical cancer cases, only due to population growth, are expected to increase from 530,000 new cases in 2008 to 770,000 new cases in 2030. This represents a $2 \%$ increase per annum in the global burden of cervical cancer that crudely balances the $2 \%$ decline observed in more developed countries. Due to the small numbers involved, it is difficult to discern temporal trends for other HPV-related cancers.

\subsection{Genital warts}

GW represents a sexually transmitted benign condition usually caused by HPV6 or 11 (some of the most methodologically rigorous studies have found HPV6/11 in 96-100\% of lesions). Although surveillance figures are difficult to obtain, data from developed countries indicate an annual incidence of $0.1-0.2 \%$, with a peak occurring at teenager and young adult ages.

GW represents a 'short incubation period HPV disease'. Therefore, if the efficacy of the quadrivalent HPV vaccine against HPV6/11 disease that was reported in the pivotal randomized, controlled trials is translated into population-based effectiveness, we may quickly see reductions in or even potentially elimination of GW.

\section{Updating the natural history of HPV and anogenital cancers [4]}

\subsection{Natural history of cervical HPV}

Cervical infections are the best understood HPV infections. Cervical HPV persistence is the known necessary event for the development of cervical precancer and cervical cancer. New infections appearing at any age are benign unless they persist. The early natural history of HPV infection and first steps of cervical carcinogenesis have been directly observed in prospective studies of the kind illustrated in Fig. 5.

A negative HPV test implies low risk of CIN3+ and a positive HPV test implies a higher risk, although many infections do clear. For HPV16, the risk of CIN3+ at 12 years of followup among women with two positive tests (at baseline and 2 years later) was $47.4 \%$ (95\% confidence interval 34.9-57.5\%). Several long-term natural history studies have now shed light on the very low risk of CIN3+ in women past the peak of HPV acquisition (e.g., 30 years or older) who are HPV-negative or who clear their HPV.

CIN2 has had been thought to be an intermediary step from CIN1 (HPV infection) to CIN3 (direct cancer precursor). More recently, the existence of this intermediary step is questioned as is the actual diagnosis of CIN2. Many studies have shown that the reproducibility of 
CIN2 is quite poor. Although CIN3 appears to have more reproducibility, several studies have found quite poor reproducibility of CIN3 as well, specifically in young women. Recent data have shown that regression rates of CIN2 among young women (defined as less than 25 years of age) are quite high (up to $75 \%$ ).

The best established etiologic co-factors for invasive cancer among HPV-infected women are smoking, long-term hormonal contraceptive use, multiparity and human immunodeficiency virus (HIV) infection.

\subsection{Heterosexual transmission and autoinoculation}

Data on transmission of HPV are finally emerging, demonstrating that transmission between heterosexual couples is extremely common, although rates vary widely among studies. Factors that affect the calculations of these rates include a) intervals between testing points, b) rates of concordance or discordance at baseline, and c) difficulty in defining established infections versus contamination. Both cervix to anus and anus to cervix autoinoculation (i.e., from one site to the other in the same woman) appears to be quite common. Whether either site serves as a long-term reservoir is unknown.

\subsection{Natural history of HPV infections in the anus in men and women}

Studies show that anal HPV infections in women and in men who have sex with men are quite common, particularly in HIV-infected individuals. Similarly, clearance of anal HPV is also common, with few individuals showing persistence unless they are HIV-infected. HIV strongly influences the development of anal intraepithelial neoplasia (AIN). The few studies on the natural history of AIN in HIV-infected men suggest that high-grade AIN is a precursor to invasive anal cancer. Although no natural history studies of AIN are available in women, women with other HPV-associated lesions, including CIN3+ and vulvar cancer, have higher rates of anal cancer.

\subsection{Natural history of penile and external genital HPV}

Data on the natural history of HPV of the male genitalia are also emerging, although penile intraepithelial neoplasia (PeIN) is poorly understood. Prevalence of penile and external genital HPV infection is greater than cervical HPV but persistence is less likely. As with genital HPV prevalence, high numbers of sexual partners increases acquisition of oncogenic HPV infections. Clearance of oncogenic HPV infection decreases in men with a higher number of lifetime female partners and is more rapid with increasing age. The seroprevalence for one or more of the quadrivalent HPV vaccine types is lower for men than for women, although the seroprevalence for any HPV vaccine type increases with age. Unlike with women, HPV prevalence is steady across all ages, suggesting that men do not develop protection against reinfection.

\section{HPV and diseases of the upper airway: head and neck cancer and respiratory papillomatosis [5]}

HPV infection is causally associated with benign and malignant diseases of the upper airway, including RRP and oropharyngeal cancer. 


\subsection{Head and neck cancers}

HPV16 definitively satisfies both molecular and epidemiologic causal criteria as a carcinogenic or high-risk type in the upper airway (Table 4). HPV16 E6/E7 mRNA expression and integration are observed predominantly among oropharyngeal cancers, and experimental models have shown E6/E7 expression to be necessary for the initiation and maintenance of the malignant phenotype of these cancers. From an epidemiologic perspective, a strong and consistent association between different markers of HPV16 exposure (LI, E6, E7 serology and HPV DNA detection in exfoliated cells) and oropharyngeal cancer has been demonstrated in numerous case-control studies. Preliminary evidence indicates that a small proportion of these cancers may be caused by additional HPV types (e.g., 18, 31, 33, 35). Associations with other anatomic head and neck cancers are neither strong nor consistent when compared to those found for oropharyngeal cancer.

HPV-positive oropharyngeal cancers have also been shown to be distinct from HPVnegative head and neck squamous cell cancers with regard to molecular genetic alterations, risk factor profiles, population-level incidence trends over time, and prognosis. Main findings are briefly summarized below:

- Molecular genetic alterations differ between HPV-positive and -negative oropharyngeal carcinomas. HPV E6 and E7 oncoproteins bind to and inactivate tumor suppressor proteins $\mathrm{p} 53$ and $\mathrm{pRb}$, respectively, leading to malignant transformation of infected cells. Since pRb is degraded, p16 expression levels in proliferating cells are high.

- Regarding interaction with other head and neck cancer risk factors, the literature is still inconsistent regarding whether tobacco/alcohol use can act as co-factors and/or effect modifiers for risk of HPV-positive oropharyngeal cancers. Some studies have shown positive associations with tobacco and/or alcohol use while others none.

- Regions of high head and neck cancer incidence include countries in Asia, where prevalence of chewing tobacco use is high, as well as parts of Central and Eastern Europe, and South America. Despite overall declines in head and neck cancer incidence in most parts of the world, recent studies from several countries-mostly developed countries (e.g., Australia, Canada, Denmark, England, Japan, The Netherlands, Norway, Sweden, USA) - have shown that the incidence of oropharyngeal cancers, including cancers of the base of the tongue, tonsil, and other parts of the oropharynx, has significantly increased, over the last 20 years. The same trend has been observed for specifically HPV-positive oropharyngeal cancers. By contrast, in most but not all of these countries, incidence of nonoropharyngeal head and neck cancer sites has significantly declined. Differences in sexual behavior, as well as in patterns of tobacco use, likely contribute to these geographic differences.

- Tumor HPV status (as determined by certain HPV16 in situ hybridization assays or certain p16 immunohistochemistry assays) is the strongest determinant of survival for patients with local-regionally advanced oropharyngeal cancer: patients with HPV-positive cancer have at least a 50\% improvement in overall survival at 5 
years, which is equivalent to an approximate $30 \%$ difference in absolute survival. Thus, HPV status determination is now part of the routine diagnostic evaluation for prognostication. However, consensus standards for specific laboratory methods and interpretation have yet to be established. It is important to highlight that the use of HPV for therapeutic decision-making outside the context of clinical trials is not yet recommended. Clinical trials for the HPV-positive patient population, the majority of whom are expected to survive, are now designed to evaluate whether the intensity of treatment and associated morbidity can be reduced without compromising survival.

\subsection{Recurrent respiratory papillomatosis}

Low-risk HPV types 6 and 11 are the predominant cause of RRP. The disease is characterized by growth of multiple papillomas, usually arising from the larynx. RRP can manifest in early childhood (juvenile onset) or in adulthood and is a rare disorder. The strongest risk factor for juvenile RRP is a maternal history of GW in pregnancy, while for adult RRP, these are lifetime number of sexual partners and oral genital sex. Many therapies have been tried for RRP, with limited success and often with severe side effects (e.g., surgical, topical, and systemic treatment with immunomodulators, antivirals, and chemotherapeutic drugs). It may be that the most effective long-term therapy will be the one that stimulates an effective, persistent immune response. Controlled trials of potential therapeutic agents are needed.

\subsection{Primary and secondary prevention of HPV-associated diseases of the upper airway}

Although detection of oral HPV DNA is associated with oropharyngeal cancer, its utility as a mechanism for secondary cancer prevention through screening is still unknown. Preliminary studies have observed no association between HPV16 DNA detection by polymerase chain reaction (PCR) in brush cytology samples and cytopathology, arguing against the potential utility of a 'Pap-smear' equivalent. Nor is there a clearly defined precancerous state or lesion in which to intervene to prevent invasive cancer.

While HPV vaccines have been shown to prevent incident and persistent anogenital infection, as well as anogenital precancers associated with HPV16 and 18, vaccine efficacy in preventing oral HPV infections has not been investigated. Observed associations between sexual behavior and both oropharyngeal cancer and adult onset RRP suggest that vaccination prior to onset of sexual behavior would be protective.

\section{The biology and life-cycle of HPVs [6]}

\subsection{Biology and life cycle of HPVs}

The interaction between papillomaviruses and their hosts is very ancient. This has lead to a balance between viral replication and immune tolerance. HPVs belong to one of five genera within the family Papillomaviridae. During evolution, HPVs have adapted to specific niches, and different papillomavirus types have different disease association and disease prevalence. 


\subsection{Normal productive life cycle}

Our knowledge of the viral life cycle refers mainly to high-risk mucosotropic HPVs and is commonly extrapolated to all HPVs. However, multiple entry pathways have been invoked, depending on the virus type studied. Activated cell division, as during wound healing, is necessary for viral DNA access to the cell nucleus. Initial viral replication in the basal cells requires E1 and E2 proteins. The role of E6 and E7 in the basal layer is uncertain, particularly for the low-risk HPVs. Deregulation of E6/E7 expression is critical in determining neoplastic grade, even in the absence of viral integration. In high-risk HPVs, E6/E7 mediate proliferation of the basal and parabasal cells, facilitating lesion growth.

Several functional differences between high- and low-risk E6 and E7 proteins regarding the cellular interactome account for increased basal cell proliferation, while for most HPVs, the E6 and E7 proteins stimulate cell cycle re-entry in mid-epithelial layers to allow for genome amplification.

\subsection{Life-cycle deregulation and cancer progression}

Expression levels of E6 and E7 increase from CIN1 to CIN3, facilitating the accumulation of cellular genetic changes (Fig. 6). Deregulation of early gene expression can follow hormonal changes or epigenetic modifications, such as viral DNA methylation. Differential methylation patterns linked to disease severity are related to changes in viral gene expression. Integration of the viral genome into the cell genome occurs in many high-grade lesions, although cancer can arise from cells exclusively containing episomes. Certain lowrisk types can occasionally be linked with cancer progression, such as in persistent RRP. Host genetic susceptibility plays an important role, such as in the WHIM syndrome (warts, hypogammaglobulinemia, infections and myelokathexis) or epidermodysplasia verruciformis. The E6 and E7 proteins of cutaneous Beta-HPVs may act by inhibiting normal DNA damage repair or apoptosis in response to sunlight.

\subsection{Lesion regression, latency and clearance}

Most cervical HPV infections are cleared, at least to the point where the viral genomes do not persist at levels that can be consistently detected by sensitive PCR assays. HPV16 infections persist on average longer than other HPV infections, and this may contribute to its association with increased cancer risk. Cellular tropism may be different for different HPVs: HPV16, 18 and 45 infect cells with potential for glandular differentiation, and abortive or semi-permissive infections in these cells may contribute to the development of adenocarcinoma. Infection by HPVs eludes the immune response by down-regulation of multiple pathways, inhibition of Langerhans cell activation, and inadequate recruitment of dendritic cells. The cellular response against GW includes an antigen-specific CD4+ Th1 response, with resolution of high-risk HPV infection requiring cross-priming of dendritic cells and T-cell infiltration. Viral DNA can persist (probably) in the epithelial stem cells at low copy numbers during latency, immunosuppression and perhaps age-related immune senescence can lead to reactivation at the site of the previous infection. 


\section{Therapy of HPV-related disease [7]}

\subsection{Current treatment of HPV-related disease}

Standard treatment for HPV-associated anogenital lesions has primarily been by surgical excision. The current treatment of chronic and neoplastic HPV-associated conditions is summarized below:

High-grade $\mathrm{CIN}$-Treatment strategies focus on eliminating the abnormal HPV-infected precancerous cells while minimizing harm to the cervical integrity. Common procedures include a loop electrosurgical excision procedure, cold knife cone biopsy, electrofulgaration, cold-coagulation and cryotherapy. Due to the relatively inexpensive infrastructure needs and the ability to perform these procedures in an outpatient setting, a loop electrosurgical excision procedure is one of the most commonly used procedures. If there are concerns about invasive disease or issues with the margins, typically a cold knife cone is the treatment standard. Cryotherapy is a treatment widely used in many countries, since it is the only option available outside of surgical settings due to its ease of use. However, due to the lack of a specimen for histopathology, the diagnosis and visualization of the lesion must be certain prior to using cryotherapy.

Cervical cancer-Early cancers (International Federation of Gynecology and Obstetrics stage la) can be treated by conisation or radical hysterectomy with excellent survival. More advanced tumors are treated with concomitant chemoradiotherapy using cisplatin-based regimens. Chemoradiotherapy of advanced cervical cancer contributes to the 66-79\% cervical cancer survival rate at 5 years. Outlook for those patients with persistent or recurrent cervical cancer following treatment is very poor.

Vulvar intraepithelial neoplasia (VIN) and vulvar cancer-The standard of care for treating VIN remains surgical excision for unifocal disease and lesions suspicious for possible invasion. However, surgical excision is not an optimal means of treating multifocal disease. The topical agent imiquimod (an immune response modifier), and photodynamic therapy (direct damage of tumor and augmentation of anti-tumor immunity) have both shown some useful efficacy ( 50-60\%) in treatment of high-grade VIN. Treatment of vulvar cancer relies on surgery for localized disease and a combination of surgery and chemoradiation for nodal metastases. In very advanced disease, chemoradiation is preferred as the sole therapy.

AIN and anal cancer-Treatments are usually ablative, using electrofulgaration, infrared coagulation, or laser ablation. Excision is reserved for those high-grade AIN where there might be microinvasive disease or cancer. There have been encouraging results from clinical trials using topical therapies for the treatment of high-grade AIN such as 5-fluoruracil (5FU), imiquimod, and topical cidofovir.

PeIN and penile cancer-For local disease, surgical treatment remains the best option. Regional disease is treated with radical inguinal lymphadenectomy if resectable; for nonresectable regional disease and metastases, neoadjuvant cisplatinum-based regimens are 
the best option. Topical chemotherapy agents (5-FU, imiquimod) are moderately effective first-line therapy in the treatment of PeIN.

Head and neck cancers-Inoperable oropharyngeal squamous cell carcinomas are treated by regimens using fractionated radiotherapy combined with cisplatin. The better relative survival of the HPV-positive patients is independent of the therapy.

GW-Provider-administered treatments of GW include cryotherapy, trichloracetic acid, or surgical removal, which has the highest primary clearance rate. Patient-applied therapies include podophyllotoxin and imiquimod. Recurrence after "successful" treatment is 30 $40 \%$.

RRP-Treatment is by surgical debridement, but many adjuvant therapies have been used with varying claims as to their effectiveness.

\subsection{Development of novel treatments}

The immune system plays an important role in controlling the development of HPVassociated cancer through specific immunity to the E6 and E7 oncogenes. Chronic high-risk HPV infection/neoplasia is characterized by systemic and/or local immune suppressive regulatory or escape factors. Recently, two E6/E7 vaccines have shown some clinical efficacy in high-grade VIN patients. Clinical response correlated with the development of HPV-specific T-cell response and with modulation of key local immune factors. Given the importance of the local microenvironment in the persistence of HPV-induced lesions and tumors, treatments that can shift the balance of immune effectors locally may be effective. A good example is the potential of imiquimod to prime the microenvironment for successful immune-mediated clearance of vulvar lesions.

Further improvements could derive from a rational combination of current and/or immune therapy with new drugs targeting molecular pathways mediated by HPV in cancer. Small molecule inhibitors targeting the DNA-binding activities of HPV E1/E2 or the anti-apoptotic consequences of E6/E7 oncogenes are in preclinical development. Proteosome and histone deacetylase inhibitors, which can enhance apoptosis in HPV-positive tumor cells, are being tested in early clinical trials. Treatments that can shift the balance of immune effectors locally in combination with vaccination are now being tested.

The challenge for immune- or antiviral-based therapies for HPV-associated conditions would be to safely provide a clear advantage over any existing treatments, especially for excisional treatments for CIN2/3 that are 90-95\% effective. It is likely that the proof-ofprinciple for therapeutic treatments will come from testing agents in the context of unsatisfactory clinical management where there is room for improvement. Such conditions include high-grade VIN, recalcitrant GW, RRP, or advanced HPV-associated cancers. The increased understanding of the role of immune regulation in limiting effective anti-tumor responses, particularly lesion infiltration of effectors and the optimal use of adjuvants in vaccines, will drive the successful development of immunotherapeutic regimens in the next 5 years. 


\section{Evidence regarding HPV testing in secondary prevention of cervical cancer [8]}

Regularly updated reviews of the full validity of screening tests have been a major benefit to clinicians as they facilitate the understanding of the continuously increasing amounts of new information regarding innovative cervical cancer prevention methods. Meta-analyses and systematic reviews have been performed on three possible clinical applications of HPV testing. These include triage of women with equivocal or low-grade cytologic abnormalities; prediction of the therapeutic outcome after treatment of CIN lesions, and last but not least, primary screening for cervical cancer and precancer.

\subsection{Triage of women with minor abnormal cytology}

Consistent evidence is available indicating that HPV triage with the Hybrid Capture ${ }^{2} 2$ assay (HC2) (Qiagen Gaithersburg, Inc., MD, USA [previously Digene Corp.]) is more accurate (higher sensitivity, similar specificity) than repeat cytology to triage women with equivocal Pap smear results. Several other tests show at least similar accuracy but mRNA testing with the APTIMA® (Gen-Probe Inc., San Diego, CA, USA) test is similarly sensitive but more specific compared to $\mathrm{HC} 2$ (Table 5, upper part).

In triage of low-grade squamous intraepithelial lesions (LSIL), HC2 is more sensitive but its specificity is substantially lower compared to repeat cytology. The APTIMA® test is more specific than HC2 without showing a loss in sensitivity. Identification of DNA of HPV types 16 and/or 18, or RNA from the five most carcinogenic HPV types allows for the selection of women at highest risk for $\mathrm{CIN} 3+$, but the sensitivity and negative predictive value of these markers are lower than full-range high-risk HPV testing (Table 5, lower part).

\subsection{Follow-up after treatment of high-grade CIN}

After conservative treatment of cervical precancer, HPV testing picks up residual or recurrent high-grade CIN more quickly than follow-up cytology, with higher sensitivity and without any decrease in specificity (Fig. 7).

\subsection{Primary screening for cervical cancer}

Primary screening for high-risk HPV generally detects more CIN2, CIN3, and cancer compared to cytology at cut-off atypical squamous cells of undetermined significance (ASCUS) or LSIL, but is less specific. Combined HPV and cytology screening provides a further small gain in sensitivity at the expense of a considerable loss in specificity, if women positive by either test are referred to colposcopy, in comparison with HPV testing only.

Randomized trials and follow-up of cohort studies consistently demonstrate a significantly lower cumulative incidence of CIN3+ and even of cancer, in women aged 30 years or older, who were, at enrollment, high-risk HPV DNA-negative compared to those who were cytologically negative (Fig. 8). The difference in cumulative risk of CIN3+ or cancer for double-negative results (cytology and HPV) versus only HPV-negative women is small. HC2, GP5+/6+PCR, cobas ${ }^{\circledR} 4800$ PCR (Roche Molecular Systems Inc., Alameda, CA, USA) and Real Time PCR (Abbott Molecular, Des Plaines, IL, USA) can be considered as 
clinically validated for use in primary screening (Fig. 9 and Fig. 10). The loss in crosssectional specificity associated with primary HPV-based screening can be compensated by appropriate algorithms involving reflex cytology and/or HPV genotyping for HPV16 or 18.

There exists a substantial evidence base to support the notion that HPV testing is advantageous both in triage of women with equivocal abnormal cytology, in surveillance after treatment of CIN lesions and in primary screening of women aged 30 years or older. However, the possible advantages offered by HPV-based screening require a well-organized program with good compliance with screening and triage policies.

\section{Nucleic acid tests for the detection of alpha HPVs [9]}

A comprehensive inventory of commercial tests for detection of alpha-HPV has identified at least 125 distinct HPV tests and at least 84 variants of the original tests (Table 6). However, only a small subset of HPV tests has documented clinical performance for any of the standard HPV testing indications. For more than $75 \%$ of HPV tests currently on the market, no single publication in peer-reviewed literature can be identified.

HPV tests that have not been validated and lack proof of reliability, reproducibility, and accuracy should not be used in clinical management. Once incorporated in the lab, it is essential that the entire procedure of an HPV test be subject to continuous and rigorous quality assurance to avoid sub-optimal, potentially harmful practices. Manufacturers of HPV tests are urged to put more effort into evaluating their current and future products analytically, using international standards, and for clinical applications, using clinically validated endpoints. To assist with analytical validation, the World Health Organization (WHO) has developed international standards for HPV types HPV16 and HPV18 and is developing standards for other types. Moreover, WHO is planning the development of external quality control panels specifically designed for use in performance evaluation of current and future HPV tests. There is a need for more competitively priced HPV tests, especially for resource-poor countries, and for uniform test validation criteria based on international standards, which should enable issuing more competitive and fair tender notices for purchasing. Automation systems allowing large-scale testing, as well as further increases in clinical performance, are the main needs in the further improvement of HPV tests.

\section{New technologies and procedures for cervical cancer screening [10]}

The clearly higher sensitivity and reproducibility of HPV DNA testing for high-grade CIN has led to widespread calls to introduce it as the primary screening test. The main concern has been its lower specificity, due to the fact that it cannot separate transient from persistent infections; only the latter are associated with an increased risk of high-grade CIN and cancer. Thus, even proponents of HPV testing generally only recommend it for women over the age of 30 years. If HPV testing is to reach its full potential, new approaches with better specificity are needed, either as triage tests for HPV-positive women or, if the high sensitivity of HPV DNA testing can be maintained, as alternative primary screening modalities. Approaches that may be useful in this regard, especially as triage tests, include HPV typing, methylation (and consequent silencing) of host and viral genes, and adding 
immunocytochemistry to cytology, such as combined dual staining for $\mathrm{p} 16^{\mathrm{INK} 4 \mathrm{a}}$ and Ki-67, which attempt to identify p16, as a surrogate for HPV, bearing proliferating cells (Table 7). At an earlier stage of development are direct methods based on detection of HPV E6 or E7 proteins.

Self-sampling for hrHPV types provides a simple alternative to attending a clinic for screening in person. Studies in both developed and developing countries have shown that self-sampling is generally preferred by the woman to clinician sampling. Studies generally show that self-sampling has a sensitivity for high-grade disease that is 10-19\% lower than clinician HPV testing. However, self and clinician collected HPV samples are more sensitive, although less specific, than cytology. Self-sampling for HPV is particularly attractive for use as the primary screening test in countries which do not have the infrastructure for clinic-based screening programs.

The need for simple, affordable cost-effective screening approaches for cervical cancer prevention in low-resource countries have led to the evaluation of visual screening methods and new screening paradigms such as low-intensity screening (single screen) and a single visit 'screen-and-treat' approach when screen-positive women, without evidence of invasive cancer, are treated with cryotherapy, without triaging procedures such as colposcopy and biopsy to minimise loss to follow-up, delay in treatment and missed disease. Visual inspection with acetic acid (VIA) is the most widely evaluated visual test whereas data for visual inspection with Lugol's iodine (VILI) are rather limited. VIA, despite all its limitations, may be used for screening premenopausal women in low-resource settings, where resources do not permit current HPV testing methods. This is the only feasible test in most sub-Saharan countries and the World Health Organization's African regional office (AFRO) has recommended its wide use in sub-Saharan African countries. VILI is not recommended as a standalone visual test. It is useful as an adjunctive to VIA.

\section{Introduction of HPV DNA screening in the world: 15 years of experience [11]}

The discovery of the necessary cause of cervical cancer, HPV, has led to important technological advances, including the development of molecular tests for HPV to identify women with cervical precancerous lesions. HPV testing has proven to be more sensitive and more reliable, albeit cross-sectionally less specific, for detection of cervical precancer than cytologic methods of detection. As the result, HPV testing can reduce the incidence of cervical cancer within 4-5 years and reduce the mortality due to cervical cancer within 8 years compared to cytology. Additionally, a negative HPV test provides greater reassurance against cervical cancer than a negative Pap test. HPV testing, because of its attributes, is useful for screening out low-risk women who do not need further intervention for 5 or more years. Thus, HPV testing can shift the emphasis of the use of Pap testing or any other more specific diagnostic test from frequent use in the entire population to the $\sim 10 \%$ subset of women who tested positive for the causal factor, HPV.

HPV testing provides an objective measure of cervical cancer risk that can be implemented effectively in high-resource and potentially lower-resource settings. An additional benefit of 
HPV testing is that rather than requiring the external quality control measures needed to maintain performance of a subjective test, such as Pap and VIA, the test already includes simplified internal quality controls.

The scalability and immediate and long-term impact of HPV testing on cervical cancer risk has been demonstrated, whereas the evidence for VIA is ambiguous and the success of cytology programs remains primarily in high-resource settings that can implement a program of frequent screens.

The exact program must be designed to meet local needs and capacities, as well as to take into consideration the local sensitivities related to cancer risk vs. over-screening and treatment. In addition, the more the community can be involved in the screening process (such as self-collection programs), the more human and financial resources that will be available for medical management of patients with positive tests.

\section{HPV vaccines - immune responses [12]}

Immune mechanisms for protection against infection and for recovery from infection are not necessarily the same; the latter is almost invariably the result of cell-mediated immunity and the recruitment and activation of cytotoxic effector cells. These may be irrelevant to the prevention of infection and disease afforded by the vaccine which, like most of the successful prophylactic vaccines used at the present, appears to be via antibody.

\subsection{Mechanisms of vaccine-induced protection}

The current assumption is that HPV virus-like particle (VLP) vaccines protect via antibody. Systemic immunization with L1 VLPs generates antibody concentrations 1-4 logs higher than in a natural infection, possibly because of high antigen concentration and delivery route that grants access to lymph nodes and spleen. There is still no immune correlate of protection against infection, and thus no indication of minimum protective antibody titers. Animal models suggest that very low antibody titers may still be protective.

Anti-L2 antibodies are cross-neutralizing, but simple protein vaccines such as L2 are poorly immunogenic compared to the L1 VLPs. Strategies such as concatenating multiple L2 proteins boost immunogenicity and show cross-protection against HPVs 16, 18, 45 and 58 pseudovirions.

\subsection{Humoral immune responses in natural genital infection}

In a natural infection, antibodies against L1 appear 6-8 months following infection in about $50-70 \%$ of infected individuals. Other viral proteins (E1, E2 and E6) do not elicit antibody responses. The IgM response occurs first and decays. The IgG1 response appears later and is more persistent. There is disagreement over the protective role of antibodies after natural infection, perhaps because of differences in assays used for detection, cut-off definitions, study design, and data analysis. 


\subsection{Humoral antibody responses induced by HPV L1 VLP vaccination}

Vaccination with HPV L1 VLPs induces high antibody titers, but the precise conformational and/or linear epitopes that are recognized are still not known. High levels of $\operatorname{IgA}, \operatorname{IgG} 3$ and IgG4 are detected, but IgG1 responses predominate. The two licensed vaccines have proved safe in over 50,000 individuals in different countries, and show seroconversion in virtually $100 \%$ of vaccinees. Antibody titers in blood reach peaks $2-3 \log$ higher than in natural infections, wane to levels several-fold lower than peak and then remain stable for years. Antibody titers are thus not a correlate of immune protection; affinity and avidity is likely to be important. Antibodies in the cervix are detectable at lower concentrations and vary with the menstrual cycle. Partial cross-protection against HPV31, HPV33 and HPV45 have been described, but although cross-neutralizing antibody concentrations are the same for both vaccines, data for the two vaccines regarding cross-protection vary.

\subsection{Therapeutic vaccines}

HPV-associated lesion regression is most often associated with cell-mediated immune responses to E2 and E6. Effector T-cell responses to E6 and E7 are weak and do not match the clinical progress of the infection. Dysplastic lesions that allow for CD8+ T cell access are likely to regress, while in persistent disease, there is low T-cell infiltration. Our knowledge of cervical $\mathrm{T}$ cell populations is still scarce, but experimental data suggest that tissue-specific immune parameters may be highly informative and that local activation of cell responses could be a therapeutic target in high-grade precancers of the cervix, vagina and anus.

Two recent clinical trials testing HPV therapeutic vaccines in patients with vulvar epithelial hyperplasia showed complete responses in around $50 \%$ of subjects during 52 weeks of follow-up, against the expected 5\% in unvaccinated individuals in the same period, despite modest systemic $\mathrm{T}$ cell responses.

Our knowledge on the cervicovaginal commensal flora, as well as virus-mediated changes in the immune milieu, is limited. These variables may be more informative than raw E6/E7 responses in blood.

\section{A review of clinical trials of HPV prophylactic vaccines [13]}

End of study analyses of the phase III trials of prophylactic HPV VLP vaccines in young women are now largely completed. Two distinct vaccines were evaluated, Gardasil@ (Merck \& Co., Whitehouse Station, NJ USA), a quadrivalent vaccine containing VLPs of types 6, 11, 16 and 18, and Cervarix ${ }^{\circledR}$ (GlaxoSmithKline Biologicals, Rixensart, Belgium), a bivalent vaccine containing VLPs of types 16 and 18 (Table 8).

\subsection{Design of phase III clinical trials}

The clinical trials were primarily designed to demonstrate efficacy in preventing incident vaccine-related HPV infection and the preneoplastic lesions caused by incident persistent infections related to vaccine HPV types. Initiation of these trials was predicated on successful completions of a series of preceding studies including development of industrial 
scale manufacturing processes, validation of type-restricted measures of antibody responses to the VLPs, and promising safety, immunogenicity and preliminary efficacy results in preclinical and early phase I/II trials. Two phase III studies, FUTURE I and FUTURE II, evaluated Gardasil ${ }^{\circ}$, and two, PATRICIA and the Costa Rica HPV Vaccine Trial (CVT), evaluated Cervarix ${ }^{\circledR}$. All of the trials were relatively large (5,500-18,500 vaccinees), blinded, randomized and controlled trials of young women (mean age 20, range 15-26) (Table 9).

\subsection{Efficacy in young women}

Both vaccines demonstrated remarkably high and similar efficacy against the vaccinetargeted types for a range of cervical endpoints from persistent infection to CIN3 in women naïve to the corresponding type at the time of vaccination. Table 10 shows efficacy estimates for the protection of young women against HPV-related genital disease by Gardasil@ in the FUTURE I and II trials, and Table 11 shows protection against cervical disease by Cervarix ${ }^{\circledR}$ in the PATRICIA trial. Gardasil ${ }^{\circledR}$ also demonstrated strong protection against GW and vulvar/vaginal neoplasia associated with the vaccine types. Cervarix ${ }^{\circledR}$ protected against vaccine-targeted anal infections in women in an end of study evaluation. The vaccines had no therapeutic effects against established infection or CIN.

\subsection{Cross-type protection}

Both vaccines showed some degree of cross-protection for both HPV infection and lesions related to non-vaccine types. Cross-protection from 6-month type-specific persistent infection from non-vaccine types was restricted to HPV 31 for Gardasil ${ }^{\circledR}$ and to HPVs 31 , $33,52,45$ and 51 for Cervarix ${ }^{\circledR}$ in the corresponding naïve cohorts (Table 12).

Concerning cross-protection against disease endpoints by Cervarix ${ }^{\circledR}$, for individual types, CIN2+ efficacy estimates with 95\% confidence intervals above zero were noted for types $31,33,45,51,52$, and 56 when HPV16/18 co-infections were included, but only for types 31 and 33 when HPV16/18 co-infections were excluded.

Cross-protection against cervical disease endpoints was also observed for Gardasil@. HPV31 was the only individual type for which significant protection against CIN2+ was observed, $70 \%$ (95\% confidence interval: 32.1-88.2).

\subsection{Efficacy in mid-adult women and males}

Gardasil® protected mid-adult women, ages $24-45$, from incident infection and CIN caused by the vaccine types (Table 13) and protected men, ages 16-26, from incident infection, GW and AIN by the vaccine types (Table 14).

\subsection{Safety}

Both vaccines exhibited excellent safety profiles in the clinical trials. Mild to moderate injection-site symptoms, headache and fatigue were the most common adverse events in Cervarix ${ }^{\circledR}$ and Gardasil ${ }^{\circledR}$ vaccinees and controls. These solicited symptoms were transient, resolved spontaneously and did not increase with number of doses. Table 15 summarizes the assessment of serious adverse events for both vaccines. 


\subsection{Immunogenicity analysis and immunobridging trials}

Both vaccines exhibited excellent immunogenicity profiles. For practical reasons, efficacy studies have not been conducted in the primary target populations of current vaccination programs, adolescent girls and boys. However, immunogenicity bridging studies demonstrating excellent safety and strong immune responses in adolescence, coupled with the documentation of durable antibody responses and protection in young adults, lead to an optimistic projection of the effectiveness of the vaccines in adolescent vaccination programs.

\subsection{Key findings from clinical trials of HPV vaccines}

The basic profiles of the two licensed HPV VLP vaccines are now well established as summarized in Table 16. Taken together, the excellent clinical trial results strongly support the potential of the vaccines as high value public health interventions and justify their widespread implementation to prevent anogenital HPV infections and their associated neoplasia. The primary focus must now be on implementation issues to maximize the rapid, effective, and cost-efficient delivery of the vaccines to those individuals that are most likely to benefit from them. By mediation of the Global Alliance for Vaccines and Immunization (GAVI) the price of the vaccines in developing countries is now significantly decreased, making them more affordable.

\section{HPV vaccine introduction - the first five years [14]}

\subsection{HPV vaccine introduction}

The availability of prophylactic HPV vaccines has provided a powerful tool for primary prevention of cervical cancer and other HPV-associated diseases. Since 2006, the quadrivalent and bivalent vaccines have each been licensed in over 100 countries. By the beginning of 2012, HPV vaccine has been introduced into national immunization programs in at least 40 countries (Table 17). Australia, the United Kingdom (UK), the United States (US), and Canada were among the first countries to introduce HPV vaccination. In Europe, the number of countries having introduced vaccine increased from 3 in 2007 to 22 at the beginning of 2012 .

While all country programs target young adolescent girls, specific target age groups vary, as do catch-up recommendations. Different health care systems and infrastructure have resulted in varied implementation strategies, with some countries delivering vaccine in schools and others through health centers or primary care providers.

Within the first 5 years after vaccines became available, few low- or middle-income countries had introduced HPV vaccine. The main reason was budgetary constraints due to the high vaccine cost. Bhutan and Rwanda implemented national immunization after receiving vaccine through donation programs in 2010 and 2011, respectively. The GAVI decision in 2011 to support HPV vaccination should increase implementation in low-income countries.

To address questions about delivery of vaccine to adolescents in low-resource countries, demonstration projects with systematic evaluation were conducted in India, Peru, Uganda, 
and Vietnam beginning in 2006. These projects found that high coverage could be achieved using a variety of delivery strategies, including school-, and health center-based strategies, as well as those coupling HPV vaccination with other health interventions (Table 18).

In general, countries with school-based delivery and publicly financed vaccine have achieved higher coverage than those with opportunistic, clinic-based or primary care-based programs. However, school-based programs are not without challenges and may be more costly. Furthermore, in low-income countries there are concerns about reaching out-ofschool children. Nevertheless, there are some clear advantages of school-based delivery for this hard to reach age group. Factors other than delivery can contribute to levels of coverage achieved, including public financing, public health promotion of vaccine and good communication strategies, organized outreach to parents, and medical profession and public acceptance. The cause of low coverage achieved in some countries is likely multifactorial.

\subsection{Post-licensure evaluation}

Evaluation of vaccination programs includes monitoring of coverage, safety, and impact. Vaccine safety monitoring is part of routine activities in many countries (Table 19). These passive monitoring systems have limitations, including reporting of events that may have occurred coincidentally following vaccination, as well as incomplete reporting. A formal evaluation of the passive surveillance system in the US, the Vaccine Adverse Event Reporting System, was conducted after over 23 million doses of quadrivalent HPV vaccine were distributed (June 2006 through December 2008). In Australia, a review of data after 6 million doses of quadrivalent vaccine were distributed did not reveal unusual patterns of reports. Similarly, in the UK, no pattern of adverse events or reason for concern was found after 4.5 million doses of bivalent vaccine had been administered.

In the US, evaluation of specific events that might be associated with vaccination is done through the Vaccine Safety Datalink (VSD), a system that evaluates adverse events in those vaccinated compared to a control group. Data were analyzed in VSD after more than 600,000 doses of quadrivalent HPV vaccine had been administered to females and raised no concerns. Post-licensure studies by the manufacturers comparing rates of adverse events in vaccinated with unvaccinated groups are ongoing or have been completed.

WHO's Global Advisory Committee on Vaccine Safety has reviewed data on HPV vaccine three times, most recently after $>60$ million doses of the quadrivalent or bivalent HPV vaccine had been distributed worldwide. The Institute of Medicine also reviewed data on quadrivalent HPV vaccine safety in 2011. All reviews show that the accumulating evidence on the safety of HPV vaccines is reassuring.

Safety evaluations are important and communication about vaccine safety is critical, as events temporally associated with vaccination can be falsely attributed to vaccination. Antivaccination efforts, in part related to concerns about safety, have been mounted in several countries.

Biologic outcomes ranging from HPV prevalence to cancer are being monitored by public health efforts in some countries. In Australia, where high coverage with quadrivalent 
vaccine was achieved soon after introduction, impact on genital warts was observed in the age group of women targeted for vaccination. Decreases were also observed among men suggesting impact of herd immunity.

\section{Understanding HPV vaccine uptake [15]}

Factors that influence HPV vaccine uptake may be viewed from the perspective of an Information-Motivation-Behavioral Skills (IMB) model analysis. The IMB model suggests that HPV vaccination information, HPV vaccination motivation, and HPV vaccine behavioral skills are fundamental determinants of HPV vaccine uptake. According to the IMB model, individuals who are well-informed about HPV vaccination, motivated to act on what they know about it, and who possess the behavioral skills required to act effectively to seek out, fund, initiate, and complete the multi-injection HPV vaccination series, are likely to successfully navigate obstacles to vaccination and achieve immune protection.

From the perspective of the IMB model, HPV vaccine information, including information about HPV infection and sequelae, and script-like, easy-to-act-upon information about HPV vaccine acquisition, is fundamental to HPV vaccine uptake. HPV vaccine uptake motivation, based on attitudes towards undergoing HPV vaccination and perceptions of social support for undergoing HPV vaccination, are a second, fundamental determinant of whether or not an individual who is well-informed about HPV vaccination will be inclined to act on what they know to receive HPV vaccination. HPV vaccination behavioral skills- skills for bringing up HPV vaccination with providers, parents, or partners, and skills for funding vaccination and adhering to a multi-injection vaccine schedule-are required to successfully navigate the potentially complex HPV vaccination process from start to finish. HPV vaccination information and HPV vaccination motivation are expected to influence HPV vaccination uptake both directly and by triggering application of complex behavioral skills that are required to initiate and complete the HPV vaccination series. The IMB analysis also specifies macro level factors may affect HPV vaccine uptake indirectly, via their impact on HPV vaccine information, motivation, and behavioral skills, or directly, via unmediated impact on HPV vaccine uptake (Fig. 11 and Fig. 12).

The IMB approach outlines a three-phase approach to interventions to promote HPV vaccine uptake. First, formative research is conducted to assess HPV vaccine related information, motivation, and behavioral skills assets and deficits in a target population. Second, targeted interventions are created on the basis of formative research to capitalize on assets in HPV vaccine related information, motivation, and behavioral skills, and to address deficits in HPV vaccine related information, motivation, and behavioral skills, in efforts to promote HPV vaccine uptake. Third, evaluation research is conducted to determine whether the targeted intervention has had intended effects on HPV vaccine related information, motivation, behavioral skills, and HPV vaccine uptake.

Research support for the IMB model analysis of factors that influence HPV vaccine uptake in developed countries, and for an IMB model approach to strengthening vaccine uptake, are discussed. 


\section{Modeling preventative strategies against HPV-related disease in developed countries [16]}

\subsection{Overview of mathematical models for decision making}

A comprehensive approach to the evaluation of HPV prevention raises a number of technical challenges. These include accurate modeling of HPV transmission within a population, the natural history of progression to cervical preneoplastic and neoplastic disease and other HPV-associated cancers, the efficacy of vaccination against different HPV types, cervical Screening test performance for various screening and triage test technologies, diagnostic and treatment processes, and screening and vaccination uptake. Models of HPV-related disease prevention can be conceptually categorized into several interacting components, as follows: (i) HPV infection, transmission and vaccination; (ii) natural history of cervical precancerous disease, invasive cervical cancer, GW and other HPV-related conditions; and (iii) cervical screening. Fig. 13 presents a broad conceptual schema for a comprehensive model of HPV disease prevention, which is designed to inform the broad structure of future models.

\subsection{Cost-effectiveness of HPV vaccination of females}

In recent years, there have been numerous economic evaluations of the vaccination of preadolescent females against HPV in developed countries, and if long duration vaccine protection is assured, these have almost universally found this intervention to be costeffective, even in the context of existing screening programs. For female only vaccination, cost-effectiveness ratios are sensitive to the duration of vaccine-conferred protection and the associated need to consider booster injection, but appear remarkably insensitive to the type of model used (static or dynamic), the outcomes included, or the assumed cost per vaccinated individual (within a feasible range).

Studies evaluating the cost-effectiveness of catch-up vaccination to older ages consistently find that this intervention is associated with a lower cost-effectiveness, because catch-up cohorts are more likely to have prior exposure to HPV infection. However, studies differ in their conclusions about the optimal age for catch-up vaccination some found the costeffectiveness ratio became unfavorable over the age of 15 years, others over the age of 18 21 years and still others over the age of 26 years.

\subsection{Cost-effectiveness of inclusion of males in population-based vaccination programs}

The inclusion of males in population-based HPV vaccination programs has potential benefits, including direct benefits to vaccinated males for protection against male HPVrelated cancers and GW, indirect benefits to both non-vaccinated females and males via increased herd immunity, and also protection of men who have sex with men. However, the return on investment of including males in existing vaccination programs will generally be lower than that of female vaccination for two reasons; firstly because the HPV-related burden of disease in males is lower than in females, and secondly, because heterosexual males derive benefits from female-only vaccination via herd immunity, particularly if coverage in females is high. Although the inclusion of males in HPV vaccination programs can be cost-effective in some circumstances, if vaccine coverage in females is less than 
about $50 \%$, increasing coverage in females is likely to provide a better return on investment than extending coverage to males, if this can be achieved.

\subsection{Choice of vaccine type}

In countries choosing to adopt only one vaccine, an important category of evaluation is the comparison of the relative cost-effectiveness of bivalent and quadrivalent vaccines. Policy decision-makers need to consider, firstly, whether protection against $\mathrm{GW}$, as well as cancer prevention is the goal of the intervention. In cost-effectiveness terms, the comparison depends upon a complex trade-off between assumptions for the duration of direct protection, the extent and duration of cross-protection, and protection against GW and RRP. In general, studies to date suggest that the bivalent vaccine would need to be approximately $22-41 \%$ cheaper per dose to have equivalent cost-effectiveness in developed countries to the quadrivalent vaccine, mainly due to protection against $\mathrm{GW}$ provided by the quadrivalent vaccine. Future evaluations will also need to consider the cost-effectiveness of a next generation nonavalent vaccine designed to protect against $\sim 90 \%$ of cervical cancers.

\subsection{Interaction between HPV vaccination and cervical screening}

A major focus for future modeling research will be evaluating the effects of vaccination on screening programs. It is expected to be several decades before HPV vaccination has a direct impact on rates of cervical cancer, but in some countries, a relatively rapid impact on rates of detected high-grade precancerous abnormalities in young women is expected. The timing of this effect will depend on several factors, including the age at which screening is initiated, the oldest age to which vaccination catch-up was conducted, and vaccination coverage in the catch-up phase. Over time, the vaccination of successive cohorts of girls will continually reduce the average lifetime risk of developing invasive cervical cancer in the population, and therefore existing screening practices will eventually become less cost-effective.

Finding cost-effective screening approaches in vaccinated populations will require consideration of new strategies. Delaying the age at which screening is initiated, increasing the screening interval, and switching to primary HPV screening are important strategies for increasing the cost-effectiveness of screening in vaccinated women. New automated platforms for primary HPV testing should drive down test costs, which will be an important consideration in maintaining the cost-effectiveness of screening in vaccinated populations. Most of these future screening options have not yet been comprehensively evaluated for cost-effectiveness in the context of HPV vaccination. The introduction of screening at longer intervals will pose substantial challenges to screening programs. The cost-effectiveness of HPV-based screening will be adversely affected if women attend more frequently than at the recommended interval, but conversely, loss to follow-up may worsen over longer rescreening intervals. Many countries organize screening on a reminder-based system; moving to proactive 'call-and-recall' systems could be evaluated as part of the consideration of HPV-based screening. The differing compliance associated with alternative methods of organizing screening has been shown to influence the effectiveness and cost-effectiveness of longer-interval approaches in modeled evaluations. Therefore, the robustness of conclusions about the cost-effectiveness of HPV screening will need to be tested against various 
assumptions about the degree of over- and under-screening that will occur in different circumstances.

The majority of cost-effectiveness evaluations of HPV vaccination have assumed that vaccine uptake will be homogenous across different subgroups in the population. However, vaccine uptake is likely to be associated with factors such as socio-economic status and sexual behavior, both of which also have been associated with participation in cervical screening. It has been demonstrated that the cost-effectiveness of combined strategies is less attractive if high-risk groups are less likely to participate in both the screening and vaccination programs. Conversely, concerns have been raised about a potential decrease in screening participation among vaccinated women. The impact of all of these effects on the cost-effectiveness of both vaccination and screening require evaluation, using the most comprehensive emerging data available.

\section{HPV, HIV and immunosuppression [17]}

The vast majority of women infected with HIV will be co-infected with HPV. Linkage studies of HIV/AIDS and cancer registries have indicated a 2- to 22-fold increase in cervical cancer in HIV-positive women compared to HIV-negative women. In addition, it has been observed that immunocompromised individuals, such as those with HIV, are also resistant to treatment of HPV-related diseases and prone to accelerated development of HPV-associated cancers.

\subsection{Prevalence of HPV types in HIV-infected women}

HIV-positive women with cervical cancer are more likely to have multiple HPV infections. However, data on the prevalence of HPV types in invasive cervical carcinoma suggest that the proportion of infection with types HPV16/18 (responsible for over 70\% of all cervical cancers) is similar in HIV-negative and HIV-positive women, suggesting that the current HPV vaccines may prevent a similar proportion of cervical cancers regardless of HIV status.

\subsection{Biological interaction between HPV and HIV}

The interaction between the two sexually transmitted infections appears to be related to the alteration in cell-mediated immunity in HIV infected persons, increased susceptibility, and possibly reactivation of latent HPV infection. Several studies have suggested that HIV is independently associated with HPV acquisition and morbidity. It is, however, unknown whether the converse relationship is also true. Some of the common sexually transmitted infections have been postulated to increase the risk of HIV acquisition, including syphilis and herpes simplex virus. Data on the potential link with HPV and HIV are limited but suggest a similar effect, i.e., that the inflammatory response evoked by HPV may solicit cells that are vulnerable to HIV infection.

\subsection{HPV in perinatally HIV-infected children and adolescents}

Adolescents perinatally infected by HIV are known to have higher rates of HPV infection. The impact of current vaccines in HIV-infected children is unknown, but one study has shown nearly $100 \%$ seroconversion, although antibody titers were lower in HIV-positive 
compared to HIV-negative children. The efficacy of HPV vaccination in HIV-positive children requires further research.

\subsection{Anal cancer screening in HIV-infected women and men}

Anal cancer incidence is greatly increased in HIV-positive individuals, particularly in HIVpositive men who have sex with men. Anal cancer has a well known precursor stage that can be detected in a variety of ways, including anal cytology and high-resolution anoscopy. Screening for anal cancer precursors is feasible; however, the impact on reduction of anal cancer remains to be demonstrated and no randomized trials have been performed.

\subsection{Options for primary prevention of HPV infection in HIV-positive individuals}

Options for prevention of HPV-associated diseases in HIV-positive individuals include HPV vaccination. There are ongoing studies on the safety, immunogenicity, and efficacy of current HPV vaccines in HIV positive-individuals and mature data are awaited. Male circumcision may be another approach to prevention of HPV transmission, which also requires further study.

\section{HPV and cancer prevention: gaps in knowledge and prospects for research, policy and advocacy [18]}

The recognition that HPV infection is the central, necessary cause of cervical cancer paved the way to new fronts of prevention via improved screening methods and HPV vaccination.

Much has been learned in all fronts, from the molecular basis of our understanding of how HPV causes disease to the health economics of preventive strategies at the individual and population levels. Progress in other areas of cancer control has yet to show the same multiand trans-disciplinary gains seen in research on HPV-associated malignancies, which is one of the unequivocal success stories in disease prevention. Yet, as an embarrassment of riches, much more research is needed to fill the gaps in knowledge that remain before we are able to reap the benefits through translation of knowledge from all fronts. Public health research on setting-specific implementation of HPV-based preventive strategies and more concerted advocacy to counter barriers facing the adoption of these strategies are likely to yield major dividends in reducing the burden of HPV-associated diseases.

\subsection{Research questions on primary prevention via immunization}

The wealth of data that have been generated by the clinical trials of the two available HPV vaccines has left many questions unanswered. Table 20 lists these questions, the associated research issues, and the underlying hypotheses that would benefit from further research and epidemiologic surveillance. Some of these questions could be addressed by post hoc exploration of completed phase III clinical trials. Partnership with industry should be sought to provide valuable insights that would aid our understanding of the role of cross-protection, the extent of protection with incomplete vaccination regimens, pan-mucosal protection against vaccine-targeted types, and anamnestic response from subsequent exposure to HPV. Likewise, the establishment of surveillance systems linking HPV vaccination registries with data from periodic HPV surveys, screening registries, and population-based cancer registries 
could provide valuable information concerning duration of protection, possible type replacement, protection against other cancers, and safety.

\subsection{Research questions on secondary prevention via screening}

Secondary prevention via screening has been successfully deployed in high-resource countries. Pap cytology is credited with having achieved substantial reductions in morbidity and mortality from this disease over the last 50 years. Yet, Pap cytology is seen as inefficient because it requires complex and costly infrastructure to ensure consistent quality, coverage, and treatment of precancerous lesions. Because of the high false-negative rate of cytology, medical guidelines have historically required annual or (at most) triennial intervals between screen visits, which overburdens the health care system of most countries. The emergence of liquid-based thin smear cytology has mitigated some of these problems and improved the efficiency for laboratories in processing cytology caseloads; in essence, however, the problems of low sensitivity, subjective interpretation of cellular morphology, and sampling errors have not been resolved.

The advent of molecular testing for HPV DNA has opened a new era of secondary prevention for cervical cancer. Over the last 15 years, a wealth of evidence from randomized clinical trials has demonstrated that these tests have much greater sensitivity and somewhat lower specificity than cytology, which would permit the lengthening of screening intervals with adequate safety. HPV testing has high reproducibility, a lower requirement for personnel training, and is amenable to using self-collected cervical samples, all attributes that would permit its deployment in large scale screening programs and in remote areas. Automation permits high-volume testing and requires minimal training for operation. On the other hand, although several commercially available assays exist, HPV testing is still costly relative to cytology. However, lengthening of screening intervals, economies of scale postimplementation in screening programs and use of multi-analyte platforms that serve a range of clinical laboratory services would largely alleviate the costs associated with HPV testing.

The lack of specialized personnel and resource infrastructure in low-resource settings has prompted the emergence of low-technology screening methods, such as VIA. This test has been extensively field-tested in low-resource regions in Africa, Asia, and Latin America, with variable results in terms of screening accuracy. VIA may be a suitable starting point to assist the establishment of screening programs in low-resource countries in connection with immediate treatment, such as cryotherapy. In such settings, it may also be used to triage women testing positive with a low-cost HPV DNA assay (WHO is currently evaluating an HPV assay system in the field that does not require running water and relies on battery power). Research on how to combine HPV testing and VIA under screen-and-treat conditions in low-resource countries is urgently needed.

In addition to HPV testing, with its different modalities for high- and low-resource settings, and VIA, other techniques show promise as screening enhancements and are the subject of intensive research. These screening enhancements include the use of immunocytochemical markers to improve the accuracy of cytology and computer-assisted pre-selection of abnormal areas in liquid-based smears. Because of their complexity, cost, and requirement for trained personnel and laboratory infrastructure, these technological enhancements can be 
implemented exclusively in high-resource areas. Table 21 summarizes the existing gaps in knowledge and areas of research interest related to the secondary prevention strategies, as well as the issues pertaining to their implementation in different settings.

\section{Upgrading public health programs for HPV prevention and control is possible in low- and middle-income countries [19]}

Cancer is an important cause of premature death in low- and middle-income countries (LMIC). Two preventive tools are available that have the potential together to sharply decrease the impact of cervical cancer in LMIC. The combination of HPV vaccination and cervical cancer screening within existing programs is possible. Although there is a great deal of concern about introducing and strengthening HPV prevention efforts in LMIC, recent projects have demonstrated feasibility. Thus, with appropriate prioritization and resources, HPV prevention can be introduced and scaled up. The integration of both screening and vaccination will save the most lives and such strategies are endorsed by many international organizations. However, some vaccine and screening programs are financed almost entirely by special externally-based programs. These more closely resemble demonstration exercises than sustainable national programs. In order for successful demonstration projects to have a broad impact on prevention, sustainable national funding based on strong commitments is essential. There may be challenges to implementing HPV prevention programs, but none should be considered insurmountable. Many LMIC have successfully adopted an HPV prevention agenda despite prevailing pessimism. Failure to act on this issue can perpetuate inequity in sexually transmitted infection and cancer prevention. Table 22 presents a summary of tips on mobilization to boost screening activities, vaccine introduction, research and evaluation and activities on health promotion and education.

\section{Implementation of HPV immunization in the developing world [20]}

\subsection{Burden of disease from HPV-related cancers in GAVI countries}

Cervical cancer is the second leading cause of cancer death in women in less developed regions of the world and the leading cause of cancer deaths in GAVI-eligible countries, where $54 \%$ of worldwide cervical cancer deaths occur. If prevention is not implemented in these countries, population growth alone will lead to a $63 \%$ increase in deaths by 2025 . Of the 530,232 new cervical cancer cases diagnosed in 2008 (9\% of all female cancers), $48 \%$ of cases $(254,374)$ occurred in GAVI-eligible countries. Cervical cancer is the leading cause of female cancer in GAVI-eligible countries, accounting for $23 \%$ of all female cancers. Agestandardized incidence rates in these countries are almost three-fold higher than in more developed regions and 1.5-fold higher than in less developed ones. Cervical cancer peak incidence in GAVI-eligible countries is observed in women aged 60-64 years, with estimated rates of 90 cases per 100,000 women 60-64 years, five-fold higher than in more developed regions estimates for this age group (Fig. 14).

\subsection{Preventing HPV infection through vaccination}

HPV vaccines are routinely used in the National Immunization Programs in most industrial countries, and the decision by the GAVI Alliance to accept applications from eligible 
developing countries for HPV vaccine support is the single most important opportunity for children in these countries to be protected against HPV-related diseases. As it has done for other vaccines, such as Haemophilus influenzae type b, rotavirus and pneumococcal conjugate vaccines, GAVI should strongly consider developing and funding a group dedicated to working on all aspects of HPV vaccine introduction in the developing world. Immunization in middle-income developing countries not eligible for GAVI support will depend on "tiered" pricing policies or regional procurement schemes to make vaccine available at prices significantly lower than those in industrial countries.

\subsection{Special considerations for HPV vaccination programming}

Immunization coverage of infants has reached high levels in many of the poorest developing countries where complementary strategies for HPV control, such as adult screening and treatment, are poorly developed. United Nations Children's Fund (UNICEF) reports that school attendance has improved over the past decades, and by $2006,84 \%$ of children in developing countries attended primary schools. It is important to consider that there still exists wide variation between countries and that girls may have lower completion rates than boys in some countries. Experience in both industrialised and developing countries shows that school-based immunization programs have often proven to be the best strategy for reaching young adolescent populations with HPV vaccine.

Immunizing young adolescents will require expansion of immunization infrastructure to reach cohorts that currently are largely unreached, but the success of school-based strategies in industrial countries and developing country demonstration projects provides hope that relatively high coverage may be achieved in many countries. Age ranges for HPV vaccination are wider and more flexible than for infant immunization, although it is advisable to vaccinate before sexual debut. Country planners must clearly define what cohort they intend to target in order to focus their strategies, resources and messaging. After deciding on the range of ages for vaccination, planners must determine whether birth date or some other indicator (such as school grade) works best for identifying eligible girls. In cultures in which birth dates are not recorded and people do not keep track of their ages, implementing vaccination by age may not work well, as PATH found in Uganda. Because HPV vaccine requires three doses over a 6-month period, timing is crucial in school-based programs to ensure that girls are fully immunized within one school year. Program planners also must consider vacation and examination schedules, and find ways to vaccinate girls who happen to not be in school on immunization days. School-based programs also require coordination between immunization and school teams-this may be new to some EPI staff.

\subsection{Acceptability issues for HPV vaccine}

Communication and advocacy strategies for HPV control need to carefully consider local cultural attitudes toward HPV-related issues. Strategies and materials must be tailored to the culture where they are delivered, and local providers and health officials must be prepared to discuss and defend the vaccine as appropriate in each culture. Audience research prior to vaccine rollout often is recommended to test messages and develop new, culture-specific information. 


\subsection{Adverse events and anti-vaccine messaging}

Anti-immunization groups spreading unfounded rumors and misinformation about vaccine safety have damaged many immunization programs, including HPV immunization efforts. Most misinformation about HPV involves rumors that HPV vaccines have caused deaths among vaccine recipients, but responsible investigation of these incidents show that no deaths have been related to the vaccine. It is critical that WHO and other groups develop messages and materials for health officials to handle anti-vaccine attacks and to provide them with the facts necessary to respond and the skills necessary to effectively deal with media.

\subsection{National decision-making for HPV immunization}

Several important factors are considered by countries when they decide whether or not to introduce a new vaccine, and how that vaccine will be used (Table 23).

Recommendations from WHO at global, regional and country levels are critical, as is the affordability of the new vaccine (and not just in the short term). Economic models of costeffectiveness and impact are increasingly important, but many ministries do not have the ability to construct these models. While some countries are comfortable relying on analyses from neighboring countries or generic models from the literature, other countries like to see data from their own country in the models before they accept them. Although some authors believe that economic models are the most important factor to consider in making these decisions, in practice the decision is influenced by a wide variety of factors.

\subsection{Considerations regarding HPV vaccination of boys}

Current strategies supported by health economic analyses call for female only immunization, but concerns have been expressed as to whether this is the optimal strategy for the developing world.

The consensus among most health economists who have modeled the cost-effectiveness and impact of male HPV immunization in the developing world is that male immunization may not be cost-effective if high coverage of females can be achieved. However, it is likely that a number of developing country immunization programs may not reach sufficiently high coverage of females. Immunization of both genders could result in further protection due to herd immunity, which could reduce transmission and protect the non-immunized. The issue here is that a strategy to immunize only females will leave high levels of circulating virus in the community and unprotected females, which in the case of rubella led to an outbreak of congenital rubella syndrome, and in the case of HPV would lead to continued numbers of cervical cancer cases. At this time, it is unclear whether female-only versus both-gender vaccinations for HPV will behave similarly to that of vaccination against rubella. That said, immunizing boys would effectively double the cohort of vaccines and significantly increase costs. GAVI has not indicated that it would provide HPV vaccine for male immunization.

Decision makers in developing countries will need to take all of these issues into consideration before deciding if male immunization is a good policy for their country. So far, WHO has recommended female-only immunization, as the major burden of the 
consequences of HPV infection affects women, but those recommendations were made before the efficacy studies on male immunization against anal precancers and external genital lesions were completed, and before much data on HPV and oropharyngeal cancer were available. The incidence of oropharyngeal cancer and extra-genital lesions in the developing world is not well documented and further study of these issues is needed.

\section{Conclusion}

When closing the ICO monograph (Vaccine Volume 30, Supplement 5, 2012) the International HPV Expert Group of editors and authors reached a consensus statement that aimed to summarize the evidence and the ways to move forward in relation to prevention of the HPV related diseases. The declaration serves us as a concluding remark of this article:

The available HPV vaccines will prevent cervical cancer and other HPV related diseases. All countries are encouraged to introduce routine HPV vaccination into their health programs and to create the conditions necessary for successful implementation.

A range of screening and treatment protocols prevent cervical cancer in women already infected with HPV. All countries are encouraged to invest in these programs and to reduce premature death from cervical cancer in women. Several screening tests exist (HPV testing, cytology, VIA). Data from clinical trials indicate that HPV based screening in women above the age of 30 is the most effective alternative.

Countries are strongly encouraged to offer well organized and coordinated vaccination and screening programs suitable to their national needs. Comprehensive efforts in cervical cancer prevention can be cost effective in a wide range of national economies.

\section{Acknowledgments}

Authors of the ICO Monograph 'Comprehensive Control of HPV Infections and Related Diseases' Vaccine Vol. 30 Suppl. 5: Ginesa Albero (Spain), Laia Alemany (Spain), Ahti Anttila (Finland), Marc Arbyn (Belgium), Lawrence Banks (Italy), Christine Bergeron (France), Jerome L. Belinson (USA), Johannes Berkhof (The Netherlands), F. Xavier Bosch (Spain), Ignacio G. Bravo (Spain), Freddie Bray (France), Thomas R. Broker (USA), Julia M.L. Brotherton (Australia), Laia Bruni (Spain), Ann Burchell (Canada), Karen Canfell (Australia), Xavier Castellsagué (Spain), Philip E. Castle (USA), Anil Chaturvedi (USA), Harrell Chesson (USA), Myriam Chevarie-Davis (Canada), Heather Cubie (Scotland), Jack Cuzick (UK), Shelley L. Deeks (Canada), Catherine de Martel (France), Lynette A. Denny (South Africa), Silvia de Sanjosé (Spain), Mireia Diaz (Spain), Joakim Dillner (Sweden), John Doorbar (UK), Mark H. Einstein (USA), Jacques Ferlay (France), Alison Fiander (UK), William A. Fisher (Canada), David Forman (France), Silvia Franceschi (France), Eduardo L. Franco (Canada), Suzanne M. Garland (Australia), Maura L. Gillison (USA), Anna R. Giuliano (USA), Marc T. Goodman (USA), Patti Gravitt (USA), Ian N. Hampson (UK), Isabelle Heard (France), Thomas Iftner (Germany), Sandra D. Isidean (Canada), Christina Jensen (Canada), Jose Jeronimo (USA), Mark A. Kane (USA), Jane J. Kim (USA), Walter Kinney (USA), Henry C. Kitchener (UK), Susanne K. Kjaer (Denmark), Boštjan J. Kocjan (Slovenia), George Koliopoulos (Greece), Shalini L. Kulasingam (USA), Charles J. Lacey (UK), D. Scott LaMontagne (USA), Eduardo Lazcano-Ponce (Mexico), Attila T. Lorincz (UK), Joannie Lortet-Tieulent (France), Lauri E. Markowitz (USA), Chris J.L.M. Meijer (The Netherlands), Anna-Barbara Moscicki (USA), Pontus Naucler (Sweden), Gina Ogilvie (Canada), Joel Palefsky (USA), Julian Peto (UK), Ligia A. Pinto (USA), Martyn Plummer (France), Mario Poljak (Slovenia), You-Lin Qiao (China), Wim Quint (The Netherlands), Guglielmo Ronco (Italy), Rengaswamy Sankaranarayanan (France), Mark Schiffman (USA), John T. Schiller (USA), Steve Schwartz (USA), Beatriz Serrano (Spain), Jennifer Smith (USA), Peter J.F. Snijders (The Netherlands), Isabelle Soerjomataram (France), Margaret Stanley (UK), Marc Steben (Canada), Bettie M. Steinberg (USA), Peter L. Stern (UK), Mark Stoler (USA), Anne Szarewski (UK), Connie Trimble (USA), Vivien Tsu (USA), Pierre van Damme (Belgium), Sjoerd H. van der Burg (The Netherlands), Andrea S. Vicari (Costa Rica), Jerome Vignat (France), Magnus von Knebel Doeberitz (Germany), Alex Vorsters (Belgium), Susan A. Wang (Switzerland), Scott Wittet (USA). 
The work was partially supported by public grants from the European Commission (7th Framework Programme grants HEALTH-F3-2010-242061, PREHDICT and HEALTH-F2-2011-282562, HPV AHEAD), from the Instituto de Salud Carlos III (Spanish Government) (grants FIS PI08/1535, FIS PI10/02995, FIS PI11/02090, FIS PI11/02096, FIS PI11/02104, RCESP C03/09, RTICESP C03/10, RTIC RD06/0020/0095, RD12/0036/0056 and CIBERESP) and from the Agència de Gestió d'Ajuts Universitaris i de Recerca - Generalitat de Catalunya (Catalonian Government) (grants AGAUR 2005SGR00695 and AGAUR 2009SGR126), who had no role in data collection, analysis or interpretation of results. Thomas R. Broker receives research support from the USPHS/NIH/ National Cancer Institute (grants "Human Papillomavirus Gene Expression" CA36200 and "Mechanisms of Human Papillomavirus DNA Replication" CA83679). Anna-Barbara Moscicki's work is supported by US Public Health Service grant R37 CA51323 (National Cancer Institute, National Institutes of Health, Department of Health and Human Services) and National Institute of AIDS and Infectious Disease RC1 AI86051. John Doorbar is funded by the UK Medical Research Council through program grant MC_U117584278 (Molecular Biology of Human Papillomavirus Infection). Marc Arbyn received financial support from: (1) the 7th Framework Programme of DG Research of the European Commission through the PREHDICT project (grant No. 242061, coordinated by the Vrije Universiteit Amsterdam, the Netherlands) and through the HPV AHEAD Network (FP7-HEALTH-2011-282562); (2) the Belgian Foundation Against Cancer (Brussels, Belgium); and (3) the International Agency for Research on Cancer (Lyon, France). Jack Cuzick was supported in part by Cancer Research UK programme grant A10404. Karen Canfell is supported by grants from the National Health and Medical Research Council, Australia (CDF APP1007994 and Project Grant \#1007518), by non-commercial government and academic consulting agreements in Australia, New Zealand and the UK, and by Cancer Council NSW, Australia. Lynette A. Denny was partially supported by Bill and Melinda Gates Foundation, USA (35537). The work of Chris J.L.M. Meijer received support via the $7^{\text {th }}$ Framework Programme of DG Research of the European commission through the PREHDICT project (grant 242061, coordinated via the Vrije Universiteit Amsterdam). Jane J. Kim is supported in part by grants from the U.S. National Cancer Institute (U54 CA164336, R01 CA160744-01A1) and the Bill and Melinda Gates Foundation (30505) for modeling of HPV and cervical cancer in developing countries.

We acknowledge editorial assistance received from Amy Yellen-Shaw (Elsevier) in the preparation of this review.

\section{Glossary}

\section{According to \\ Protocol (ATP) \\ cohorts}

\section{Bivalent HPV \\ vacane}

Dynamic model

Human

development index (HDI)

InformationMotivation-
Also designated Per Protocol Efficacy (PPE). ATP analyses are restricted to individuals who adhere to all aspects of the study protocol: for example, they received the three vaccine doses within specified intervals, and events are not counted until after receiving all three doses. Importantly, individuals included in ATP cohorts have no evidence of exposure to the vaccine targeted type under analysis. Thus ATP analyses can be viewed as the best case scenario for the effectiveness of a prophylactic vaccine

Cervarix ${ }^{\circledR}$ (GlaxoSmithKline Biologicals, Rixensart, Belgium). Contains virus like particles (VLPs) of HPV types 16 and 18

In this model the HPV transmission in the population is directly simulated: the probability of being infected with HPV depends on the number of sexual partners (often modeled as being dependent on age and behavioral subgroup), the probability of a new partner being infected, and the probability of HPV being transmitted from an infected to uninfected partner. These models capture the effects of herd immunity

The HDI is a country-specific, composite index based on life expectancy, literacy, access to education and per capita gross domestic product

A well validated approach to the prediction and promotion of health behavior performance that has been applied to HPV vaccine uptake 


\section{Behavioral Skills \\ (IMB) model}

Intention to Treat

(ITT) cohorts

ITT naive cohorts

\section{Modified \\ Intention-To- \\ Treat (MITT) \\ Per Protocol \\ Efficacy (PPE) \\ Population \\ attributable \\ fraction (PAF)}

\section{Quadrivalent \\ HPV vaccine}

Static model
Also designated Total Vaccine Cohort (TVC), include all individuals that are randomized and participate in the trial. For vaccine trials "participation" is usually defined as receiving at least one dose of the vaccine. These cohorts include women with evidence of prior HPV exposure and hence current infection/lesions by vaccine targeted as well as other HPV. ITT analyses can be viewed as an approximation of the effectiveness of the vaccine in general use, at least for individuals with similar demographic and risk characteristics as the subjects in the trial

Also designated TVC naive or Unrestricted susceptible population. These cohorts include all participating individuals with no evidence at baseline of cervical cytology abnormalities, prevalent infection by any of the genital HPV types evaluated (up to 14 types) or serological evidence of past exposure to the vaccine targeted types. These cohorts are currently the best approximation for the primary target group for the vaccines, pre and early adolescent girls who have not yet become sexually active

MITT analyses fall somewhere in between ITT and ATP, allowing for some deviation from the ideal protocol. One interesting MITT cohort is designated TVC-naïve or ITT-naïve

See According to Protocol (ATP) cohorts

The PAF is defined as the proportion of new cancer cases in a population that would have been prevented following a hypothetical intervention that would completely prevent HPV exposure. For all HPV-related cancers, the authors considered that the detection by PCR of high-risk HPV DNA in tumor tissue signifies that the cancer is attributable to HPV. Hence, the regular formulas for attributable risk calculation using a measure of the prevalence in the population and an estimate of the relative risk were not used to compute PAF in HPV-related sites. Instead, the prevalence of high-risk HPVs in cancer cases was considered a good approximation to the PAF

Gardasil ${ }^{\circledR}$ (Merck \& Co., Whitehouse Station, NJ USA). Contains virus-like particles of HPV types 6, 11, 16 and 18

In their simplest form, cohort models simulate a single birth cohort of people through their lives. In these models, the probability of incident infection is usually modeled as being dependent on age, but age-specific infection does not change overtime. Therefore, such models can only be used to estimate the direct effects of vaccination 
Total Vaccine

Cohort (TVC)

TVC-naïve

cohorts

\section{Unrestricted \\ susceptible \\ population}

Vaccine Adverse

Event Reporting

System (VAERS)

Vaccine Safety

Datalink (VSD)

Virus-like

particles (VLPs) on vaccinated groups and they cannot capture the changing effect of vaccination over time in reducing the probability of HPV exposure in unvaccinated groups, which is known as herd immunity. As a result, cohort models tend to underestimate the overall effectiveness of HPV vaccination. A commonly used static implementation is the Markov cohort model

See Intention-to-Treat (ITT) cohorts

See ITT-naïve cohorts

See ITT-naïve cohorts

A system of passive surveillance for adverse events associated with vaccination in the United States. Similar systems exist across many countries worldwide (e.g., Australia, Canada, UK)

A system in the United States that evaluates adverse events in those vaccinated compared to a control group in the post-licensure setting Particles that contain certain proteins from the outer coat of a virus. Virus-like particles do not contain any genetic material from the virus and cannot cause infection. Currently available HPV vaccines are both composed of HPV L1 proteins that spontaneously self assemble into VLPs

\section{References}

1. Broker TR. Global prevention and management of human papillomavirus related diseases: the pressing challenges and the compelling opportunities. Vaccine. 2012; 30(S5):7-10.

2. Bosch FX, Tsu V, Vorsters A, Van Damme P, Kane MA. Reframing cervical cancer prevention. Expanding the field towards prevention of human papillomavirus infections and related diseases Vaccine. 2012; 30(S5):F1-11.

3. Forman D, de Martel C, Lacey CJ, Soerjomataram I, Lortet-Tieulent J, Bruni L, et al. Global burden of human papillomavirus and related diseases. Vaccine. 2012; 30(S5):F12-23. [PubMed: 23199955]

4. Moscicki A-B, Schiffman M, Burchell A, Albero G, Giuliano AR, Goodman MT, et al. Updating the natural history of human papillomavirus and anogenital cancers. Vaccine. 2012; 30(S5):F24-33. [PubMed: 23199964]

5. Gillison ML, Alemany L, Snijders PJF, Chaturvedi A, Steinberg BM, Schwartz S, et al. Human papillomavirus and diseases of the upper airway: head and neck cancer and respiratory papillomatosis. Vaccine. 2012; 30(S5):F34-54. [PubMed: 23199965]

6. Doorbar J, Quint W, Banks L, Bravo IG, Stoler M, Broker TR. The biology and life-cycle of human papillomaviruses. Vaccine. 2012; 30(S5):F55-70. [PubMed: 23199966]

7. Stern PL, van der Burg SH, Hampson IN, Broker TR, Fiander A, Lacey CJ, et al. Therapy of human papillomavirus-related disease. Vaccine. 2012; 30(S5):F71-82. [PubMed: 23199967]

8. Arbyn M, Ronco G, Anttila A, Meijer CJLM, Poljak M, Ogilvie G, et al. Evidence regarding human papillomavirus testing in secondary prevention of cervical cancer. Vaccine. 2012; 30(S5):F88-99. [PubMed: 23199969] 
9. Poljak M, Cuzick J, Kocjan BJ, Iftner T, Dillner J, Arbyn M. Nucleic acid tests for the detection of alpha human papillomaviruses. Vaccine. 2012; 30(S5):F100-6. [PubMed: 23199952]

10. Cuzick J, Bergeron C, von Knebel Doeberitz M, Gravitt P, Jeronimo J, Lorincz AT, et al. New technologies and procedures for cervical cancer screening. Vaccine. 2012; 30(S5):F107-16. [PubMed: 23199953]

11. Castle PE, de Sanjosé S, Qiao Y-L, Belinson JL, Lazcano-Ponce E, Kinney W. Introduction of human papillomavirus DNA screening in the world: 15 years of experience. Vaccine. 2012; 30(S5):F117-22. [PubMed: 23199954]

12. Stanley M, Pinto LA, Trimble C. Human papillomavirus vaccines - immune responses. Vaccine. 2012; 30(S5):F83-7. [PubMed: 23199968]

13. Schiller JT, Castellsagué X, Garland SM. A review of clinical trials of human papillomavirus prophylactic vaccines. Vaccine. 2012; 30(S5):F123-38. [PubMed: 23199956]

14. Markowitz LE, Tsu V, Deeks SL, Cubie H, Wang SA, Vicari AS, et al. Human papillomavirus vaccine introduction - the first five years. Vaccine. 2012; 30(S5):F139-48. [PubMed: 23199957]

15. Fisher WA. Understanding human papillomavirus vaccine uptake. Vaccine. 2012; 30(S5):F14956. [PubMed: 23199958]

16. Canfell K, Chesson H, Kulasingam SL, Berkhof J, Diaz M, Kim JJ. Modeling preventative strategies against human papillomavirus-related disease in developed countries. Vaccine. 2012; 30(S5):F157-67. [PubMed: 23199959]

17. Denny LA, Franceschi S, de Sanjosé S, Heard I, Moscicki AB, Palefsky J. Human papillomavirus, human immunodeficiency virus and immunosuppression. Vaccine. 2012; 30(5S):F168-74. [PubMed: 23199960]

18. Franco EL, de Sanjosé S, Broker TR, Stanley MA, Chevarie-Davis M, Isidean SD, et al. Human papillomavirus and cancer prevention: gaps in knowledge and prospects for research, policy, and advocacy. Vaccine. 2012; 30(S5):F175-82. [PubMed: 23199961]

19. Steben M, Jeronimo J, Wittet S, LaMontagne DS, Ogilvie G, Jensen C, et al. Upgrading public health programs for human papillomavirus prevention and control is possible in low- and middleincome countries. Vaccine. 2012; 30(S5):F183-91. [PubMed: 23199962]

20. Kane MA, Serrano B, de Sanjosé S, Wittet S. Implementation of human papillomavirus immunization in the developing world. Vaccine. 2012; 30(S5):F192-200. [PubMed: 23199963] 


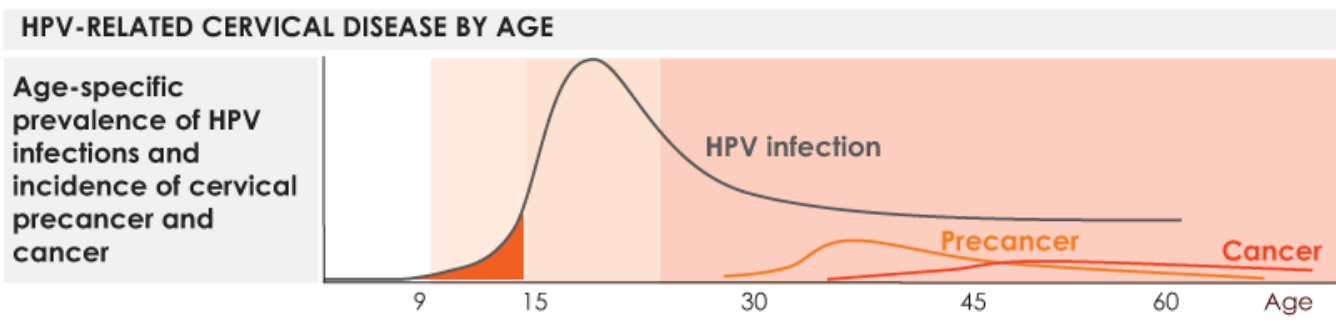

CERVICAL CANCER PREVENTION STRATEGIES

\section{Vaccines}

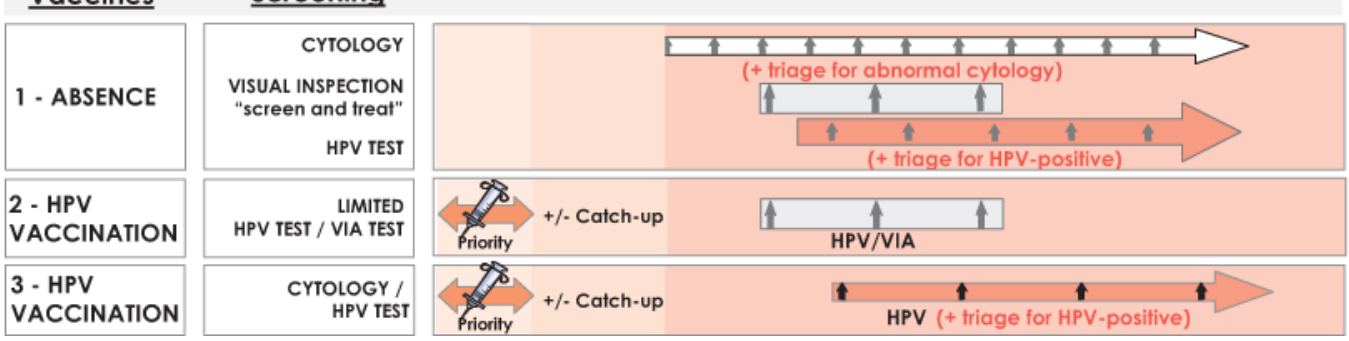

Figure 1.

Natural history and HPV-based prevention strategies according to age. VIA: Visual inspection with acetic acid. Reproduced with permission from Bosch FX et al. [2]. 


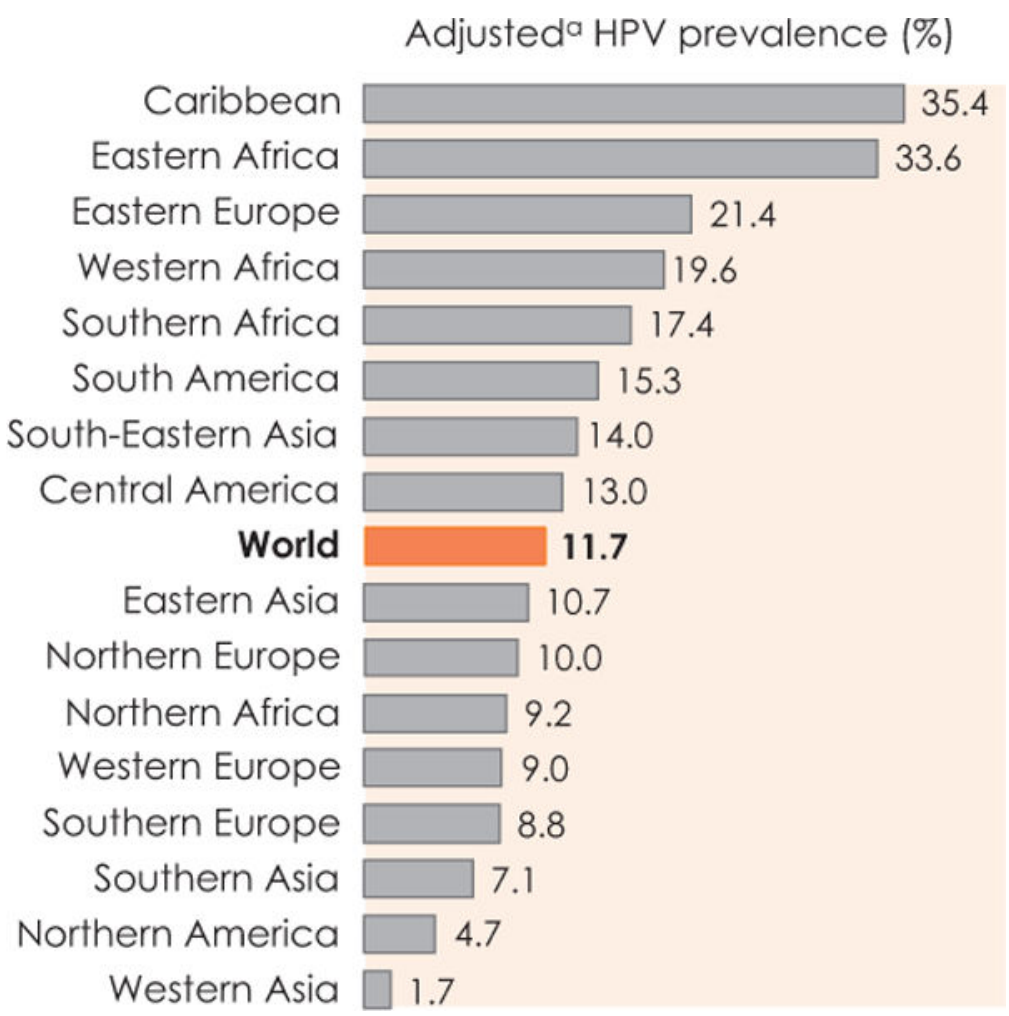

Figure 2.

HPV prevalence among women with normal cytology: meta-analysis based on results from 1,016,719 women. a Regionally-adjusted HPV (see Bruni L et al. J Infect Dis 2010;202:1789-99 for adjustment methodology). Reproduced with permission from Forman D et al. [3]. 

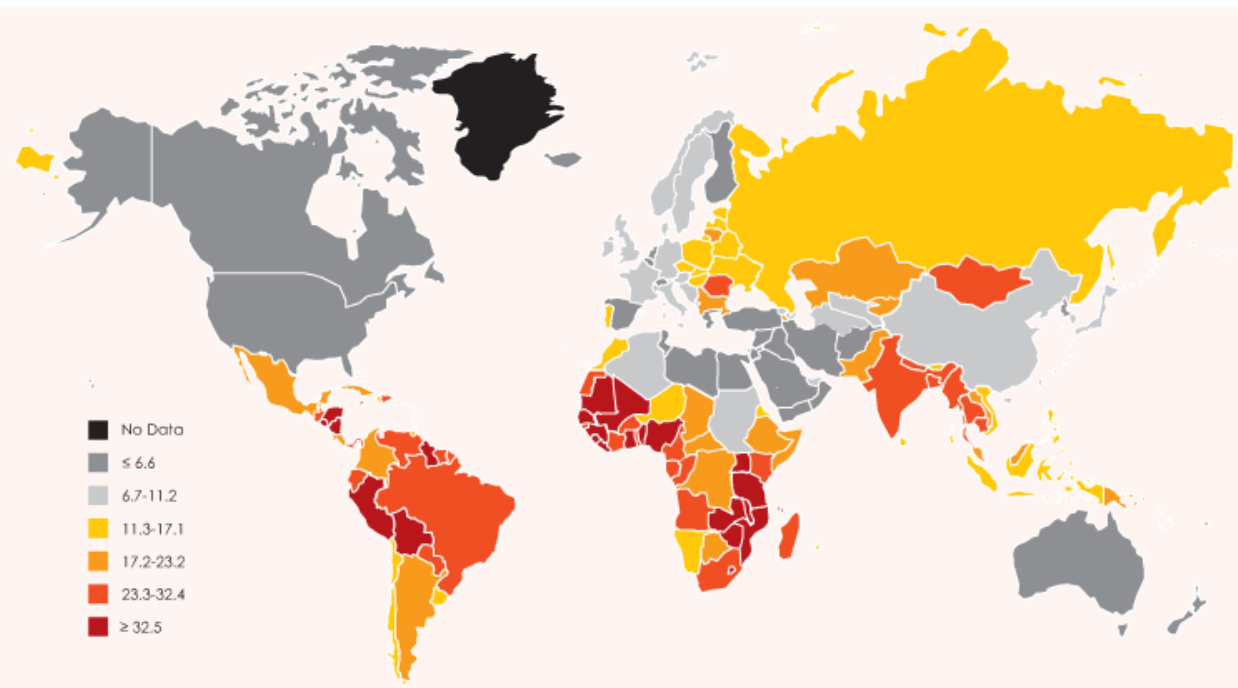

Figure 3.

Cervical cancer, global map showing estimated age-standardized (world standard) incidence rate per 100,000 in 2008 (all ages). Based on GLOBOCAN 2008. Reproduced with permission from Forman D et al. [3]. 

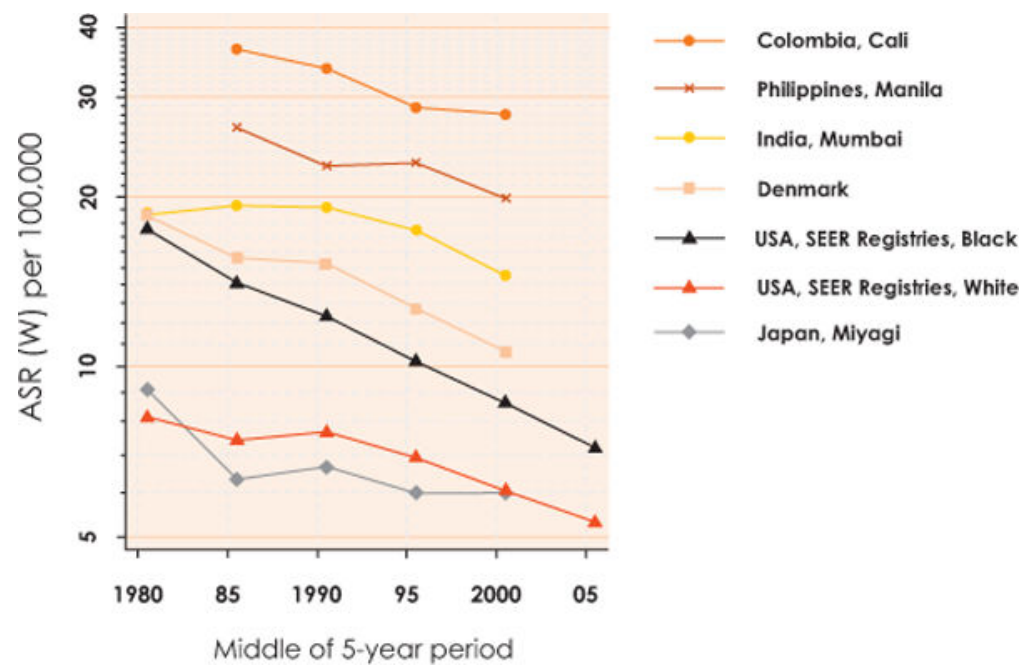

Figure 4.

Cervical cancer, age-standardized (world standard) incidence rates per 100,000, 1978-2007, per 5-year period, in selected cancer registry populations (all ages). Based on Cancer Incidence in Five Continents, Volumes V to IX and Surveillance, Epidemiology, and End Results (SEER) Program. ASR (W): Age-standardized (world standard) rate. Reproduced with permission from Forman D et al. [3]. 


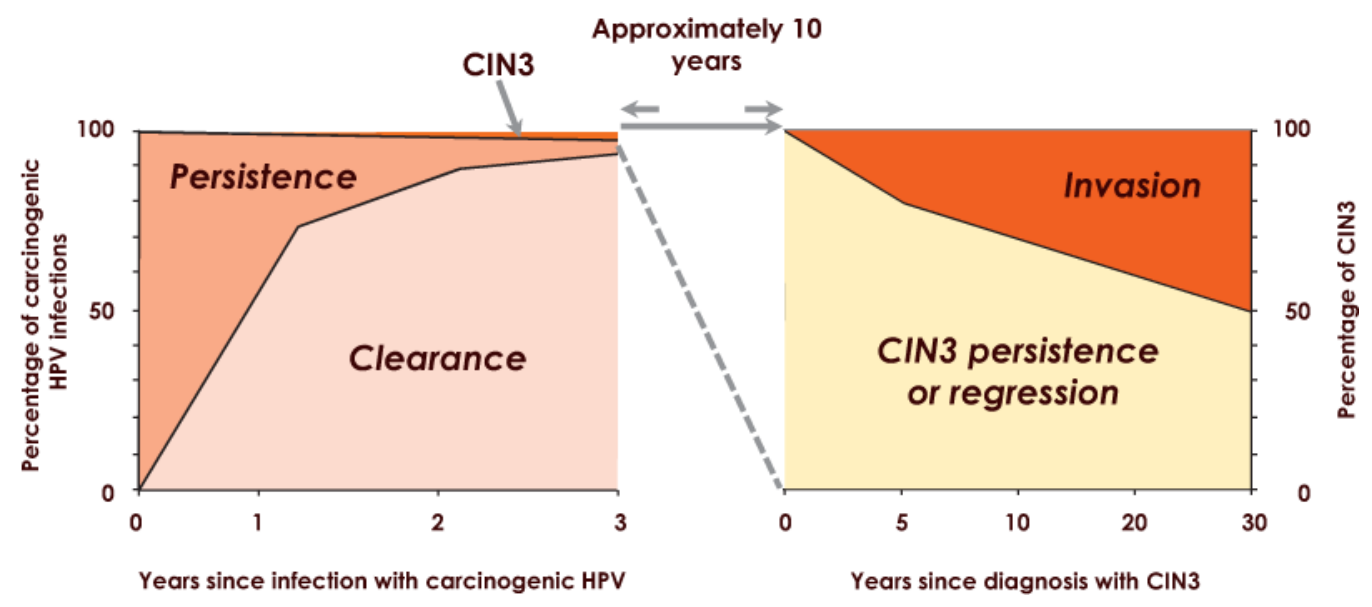

Figure 5.

Natural history of HPV infection. CIN3: Cervical intraepithelial neoplasia grade 3. Reproduced with permission from Moscicki A-B et al. [4]. 

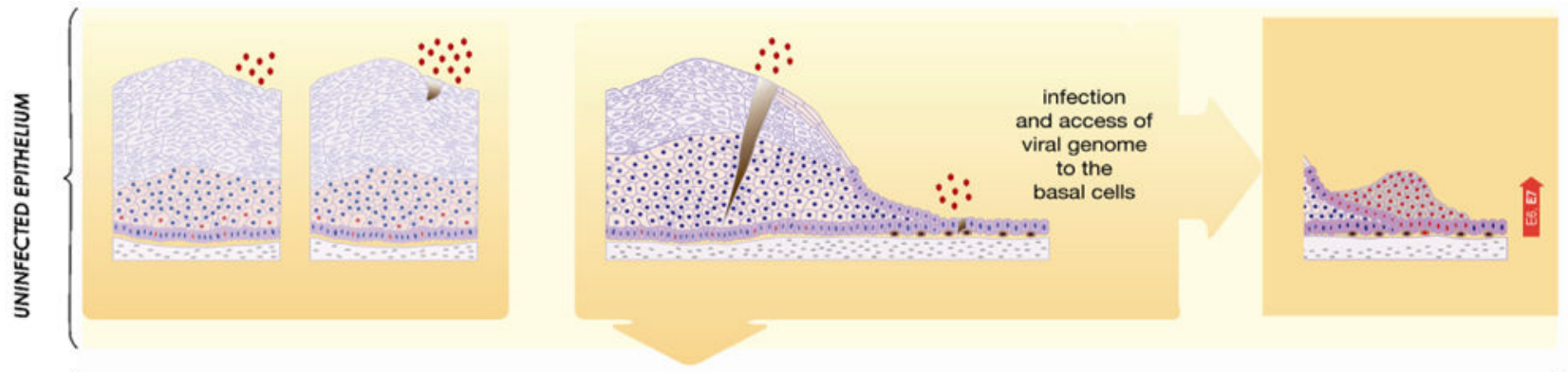

(ii)
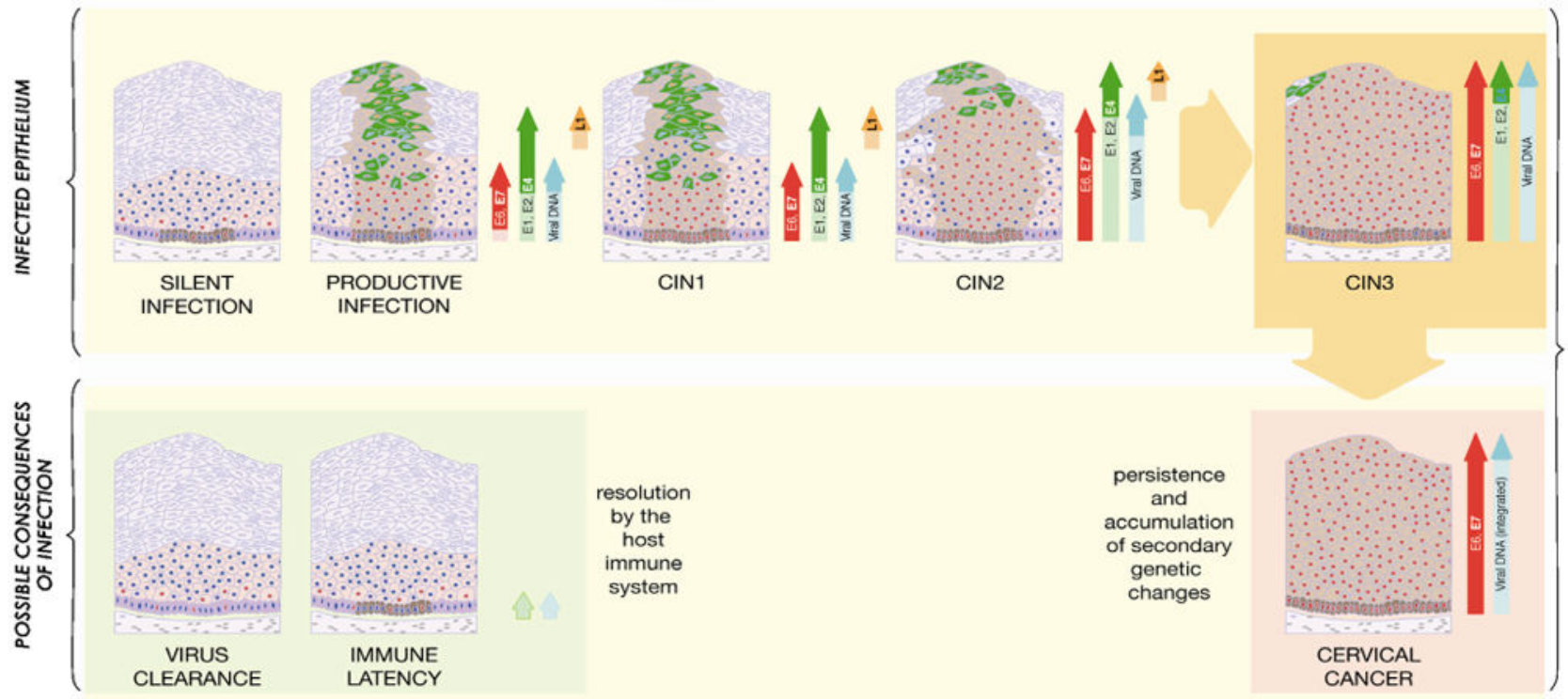

CANCER

Figure 6.

High-Risk HPV Infection and its Possible Consequences.

(i) The detection of HPV DNA in a tissue biopsy or in exfoliated cervical cells may indicate infection (productive (CIN1) or abortive (CIN3) as shown in (ii)), the presence of virus particles at the epithelial surface without infection (e.g. from recent transmission), or a latent or silent infection (as shown in (ii)). To resolve this ambiguity, markers of viral gene expression (such as mRNA or proteins) are useful in confirming the presence of active disease when HPV is detected using DNA-based tests. Infection requires the entry of HPV virions into the mitotically active epithelial cells of the basal layer, which in stratified epithelium is thought to require a microwound. In the columnar cell layers, infection is thought to be facilitated by the proximity of the target cell to the epithelial surface, which may allow the virus to access a cell type that is unable to support the full productive life cycle (right). The significance of infection of different cell types remains to be properly assessed.

(ii) Following infection (shown in (i)), expression from the viral genome can sometimes be suppressed (e.g., by genome methylation), leading to a 'silent' infection in which the viral genomes are retained in the basal layer without apparent disease. Infection may alternatively lead to an ordered pattern of viral gene expression leading to virus synthesis and release from the upper epithelial layers (productive infection or CIN1), or to deregulated viral gene expression and high-grade neoplasia (CIN2/CIN3). Persistent high-grade disease such as 
CIN2 and 3 is associated with a increasing risk of genome integration into the host cell chromosome and progression to cancer. Cells in cycle are indicated by the presence of red nuclei. Cells expressing E4 are shown in green, while those expressing L1 are shown in yellow. The brown shading on the diagrammatic representations of the epithelium identify all the cells (differentiated and un-differentiated) that contain viral genomes.

(iii) In most cases, HPV infections are resolved as a result of a cell-mediated immune response (left). This may lead to viral clearance or to viral latency and the persistence of viral episomes in the epithelial basal layer without life-cycle completion. Viral gene expression patterns during latency are not well characterised (E1, E2 expression postulated here as suggested from animal models). Persistent deregulated gene expression, as occurs in CIN3 and following viral genome integration, can lead to the accumulation of secondary genetic changes in the infected host cell and development of cancer. This is facilitated by over-expression of the high-risk E6 and E7 proteins. Cells in cycle are shown by red nuclei. Brown shading in the immune latency state indicates cells harbouring viral episomes. In cervical cancer, the viral genome is often integrated with loss of expression of full-length E1, E2, E4 and E5, and the L1 and L2 capsid proteins, and with de-regulated expression of E6 and E7. Reproduced with permission from Doorbar J et al. [6]. 

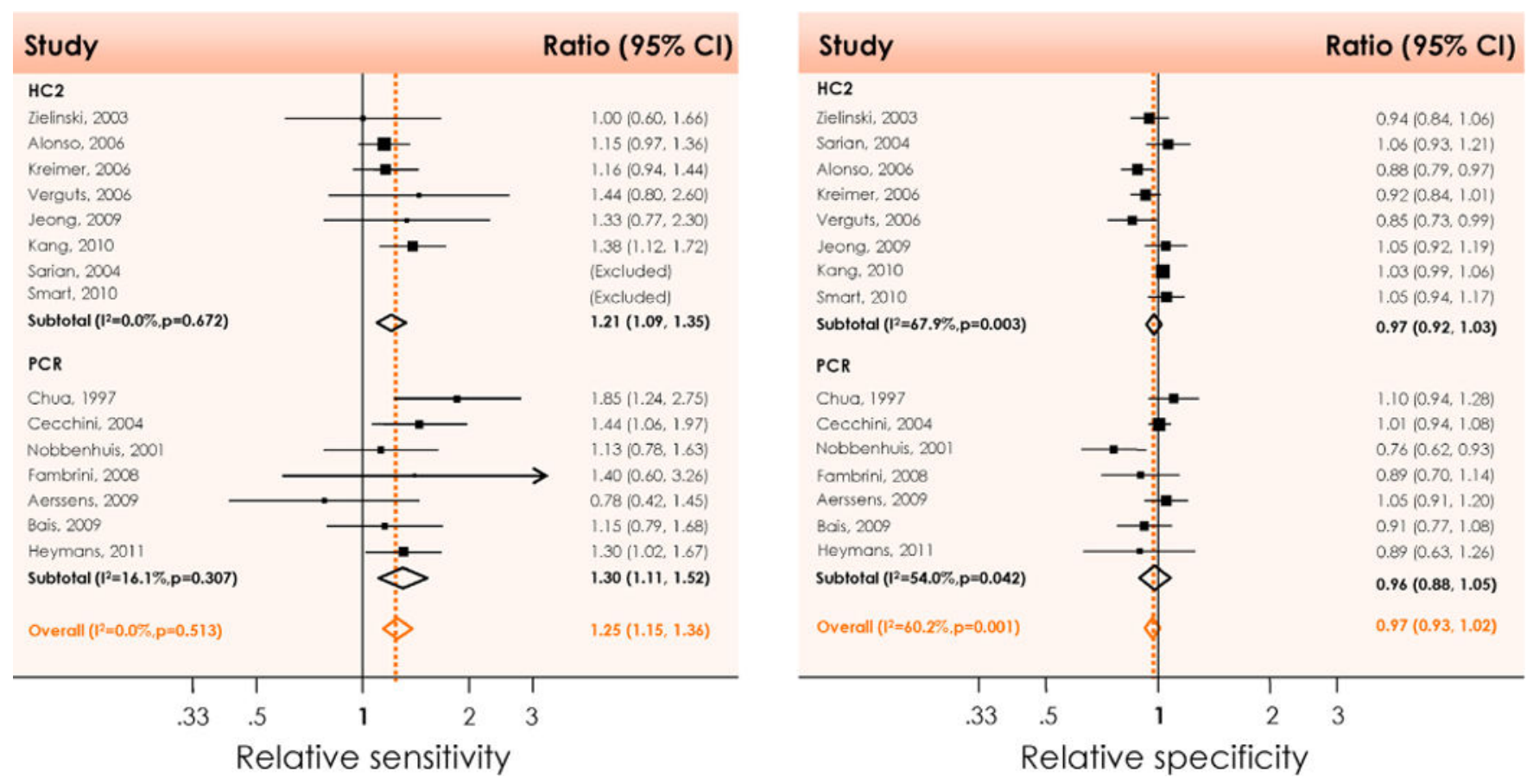

Figure 7.

Relative sensitivity (left) and specificity (right) of high-risk HPV DNA testing with HC2 or PCR compared to cytology after treatment of high-grade to predict therapeutic failure (residual of recurrent CIN2 or worse). CI: Confidence interval; HC2: Hybrid Capture® 2; ${ }^{2}$ : Percentage of total variation across studies due to heterogeneity; $\mathrm{p}$ : Test for inter-study heterogeneity; PCR: Polymerase chain reaction. Reproduced with permission from Arbyn M et al. [8]. 


\section{CIN3+}

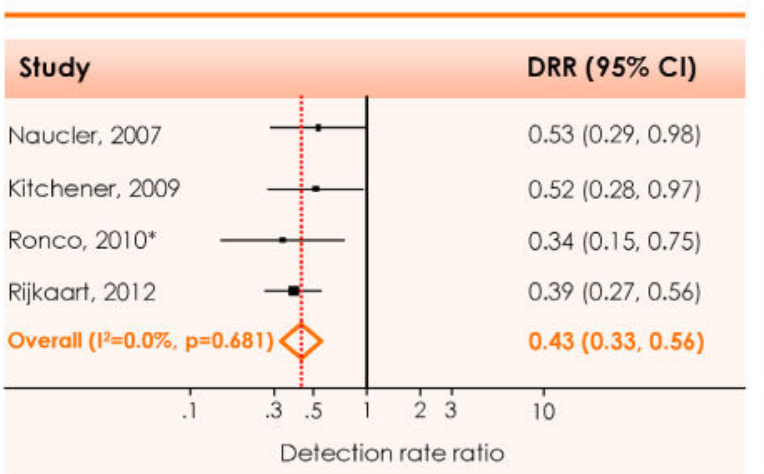

\section{CERVICAL CANCER}

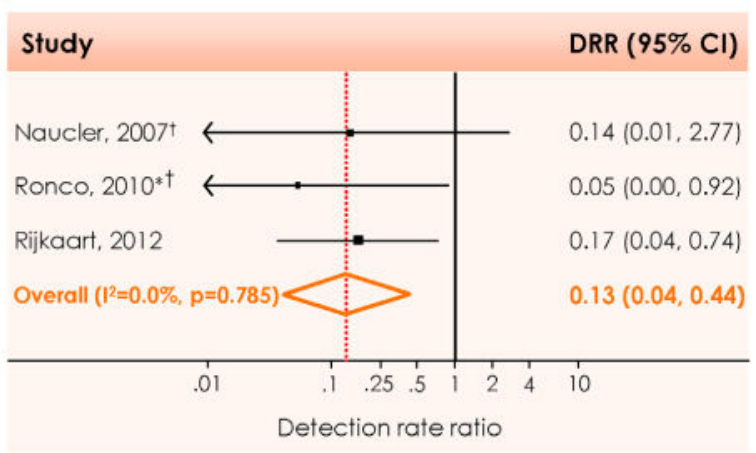

* restricted to women of 35 years or older.

t continuity correction (+.5 in each cell because of zero cancer cases among HPV-negative women).

Figure 8.

Meta-analysis of the main outcomes from randomized trials comparing HPV- and cytologybased cervical cancer screening. Relative detection rate of CIN3+ (left panel) and cervical cancer (right panel), observed in the second screening round among women who were HPVnegative versus cytology-negative at enrolment. CI: Confidence interval; CIN: Cervical intraepithelial neoplasia; DRR: Detection rate ratio; $\mathrm{I}^{2}$ : the percentage of total variation across studies due to heterogeneity; p: test for inter-study heterogeneity. Reproduced with permission from Arbyn M et al. [8]. 
HC2, OUTCOME CIN2+

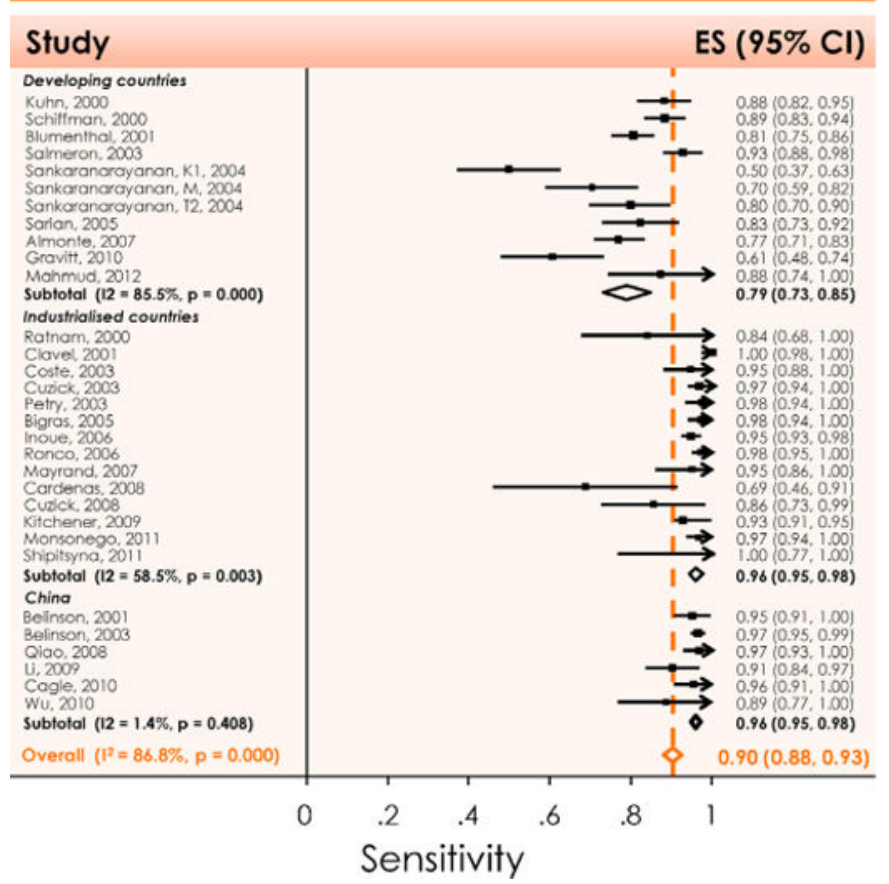

HC2, OUTCOME CIN3+

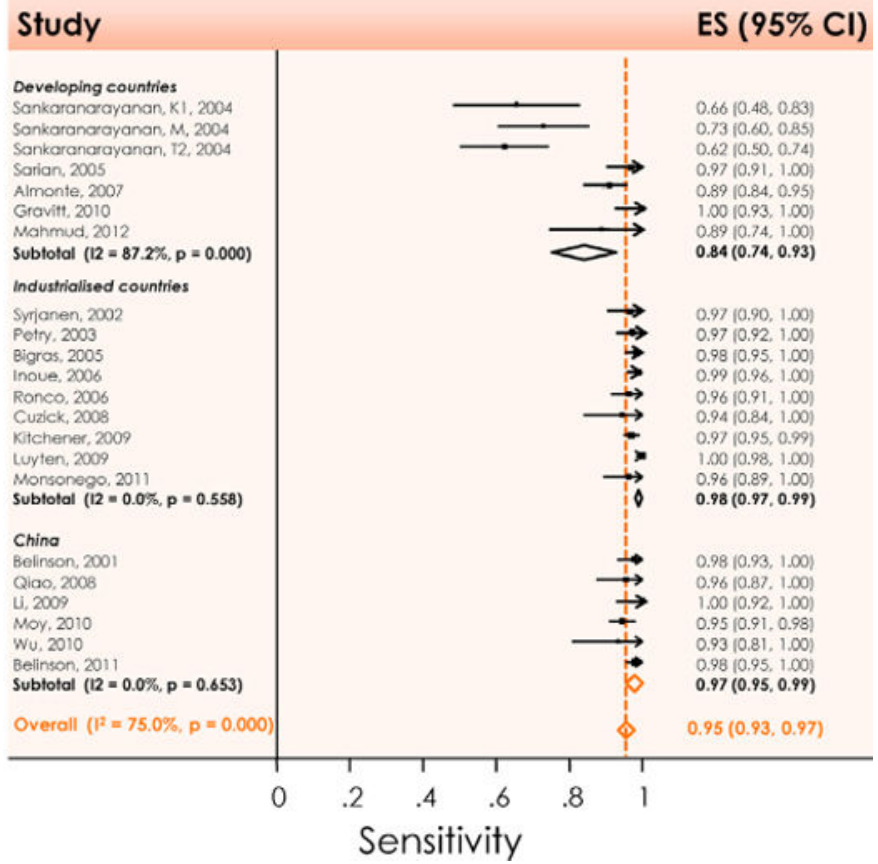

Figure 9.

Meta-analysis of the sensitivity of $\mathrm{HC} 2$ as a primary screening test to detect CIN2+ (left) or CIN3+ (right) in developing countries, industrialised countries, and China. It shows clearly that the sensitivity is very heterogeneous in developing countries (probably due to quality of gold standard), much less heterogeneous in industrialised countries and not heterogeneous in China (improved gold standard verification). CIN: Cervical intraepithelial neoplasia; ES: Estimate of sensitivity; HC2: Hybrid Capture ${ }^{\circledR} 2$; $\mathrm{I}^{2}$ : Percentage of total variation across studies due to heterogeneity; p: Test for inter-study heterogeneity. Reproduced with permission from Arbyn $\mathrm{M}$ et al. [8]. 


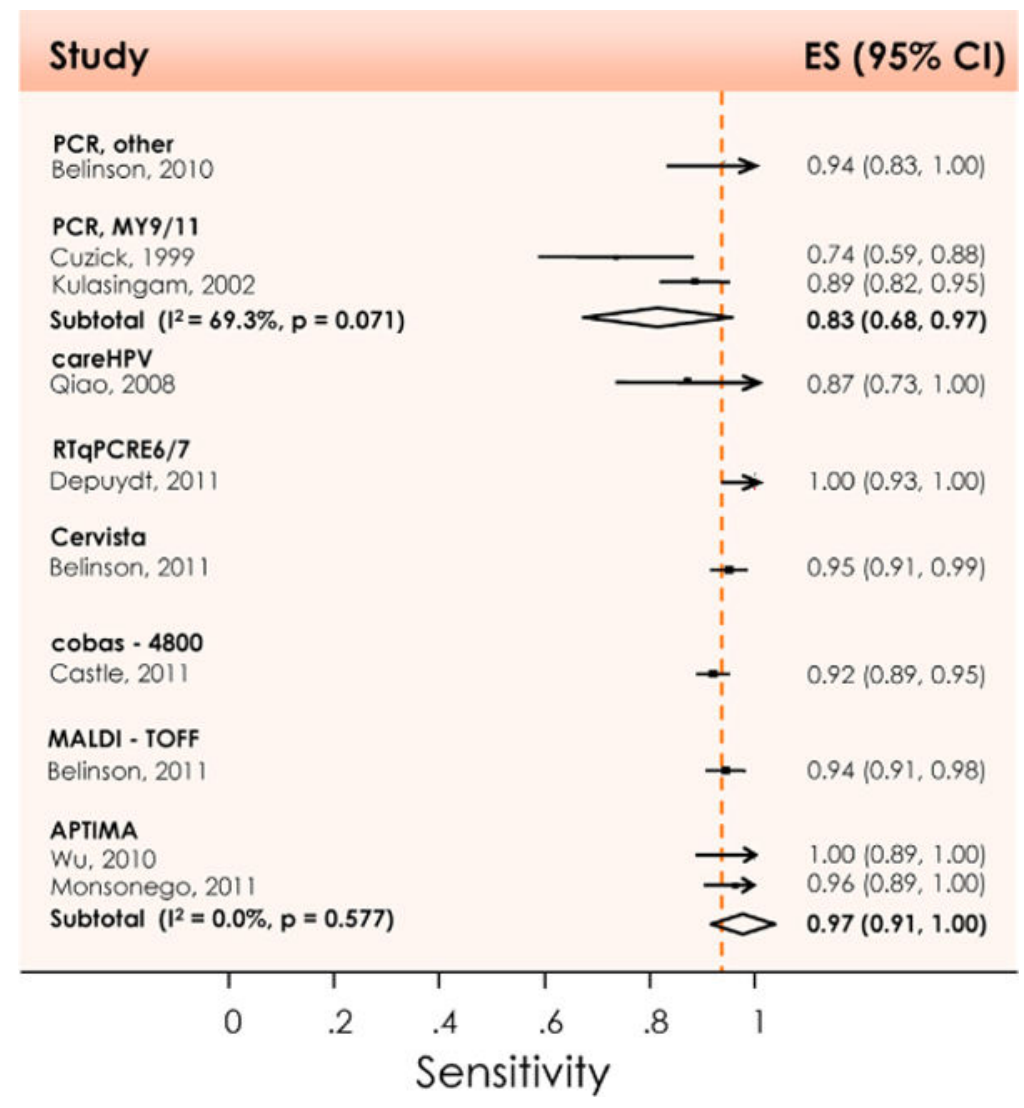

Figure 10.

Meta-analysis of the sensitivity for detecting CIN3+ in primary cervical cancer screening, using other tests than $\mathrm{HC} 2$, by test system. CI: Confidence interval; ES: Estimate of sensitivity; $\mathrm{I}^{2}$ : Percentage of total variation across studies due to heterogeneity; $\mathrm{p}$ : Test for inter-study heterogeneity. Reproduced with permission from Arbyn M et al. [8]. 


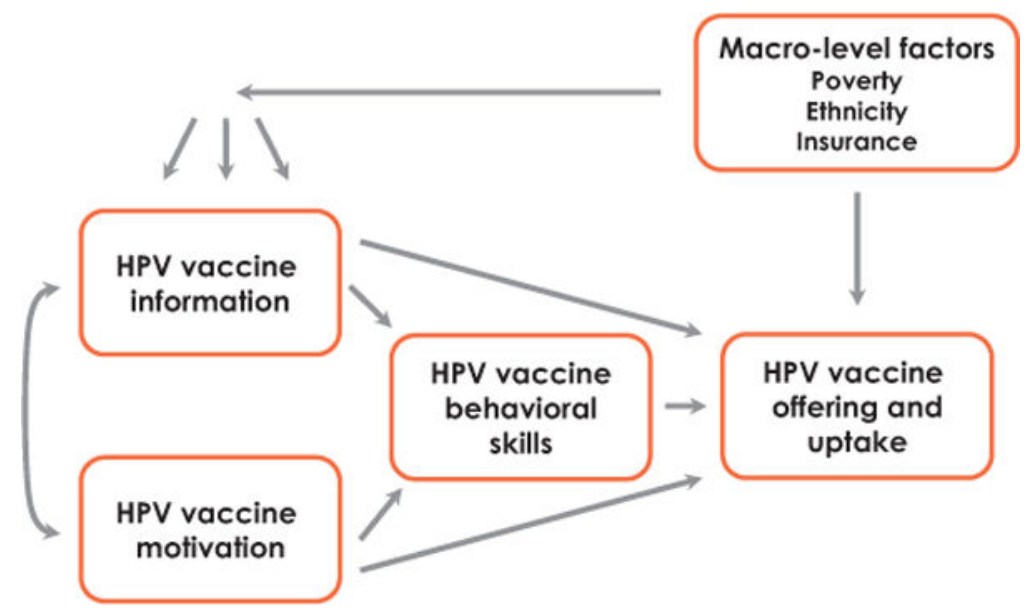

Figure 11.

An Information-Motivation-Behavioral Skills (IMB) model of HPV vaccine offering and uptake. The IMB model specifies that HPV vaccine information and HPV vaccine motivation work through the application of HPV vaccine behavioral skills to influence HPV vaccine offering (on the part of health care providers) and uptake (initiation and completion of an HPV vaccination series). In situations in which the behavioral skills demands of HPV vaccination are minimal, there may be direct associations of HPV vaccine information, HPV vaccine motivation, and HPV vaccine offering and uptake. Reproduced with permission from Fisher WA [15]. 


\section{A}

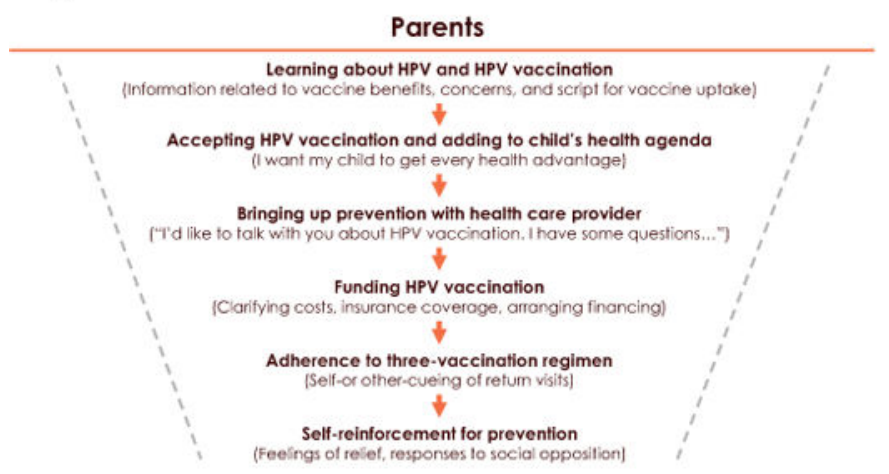

Adolescent young adult and adult women and men

Learning about HPV infection, HPV sequelae, and HPV vaccination (Easy fo franslale into action information)

Accepting HPV vaccination and adding it to one's own health agenda (I am legitimalely sexual and wish lo gain and maintain my health) $\downarrow$ Bringing up prevention with health care provider
["I'd Ike to tak with you about HPV vaccination"]

Funding HPV voccination (Clarifying costs. insuronce Adherence to three-vaccination regimen [Self-or other-cueing fo return visits) Sell-reinforcement for prevention (Feeings of reief)

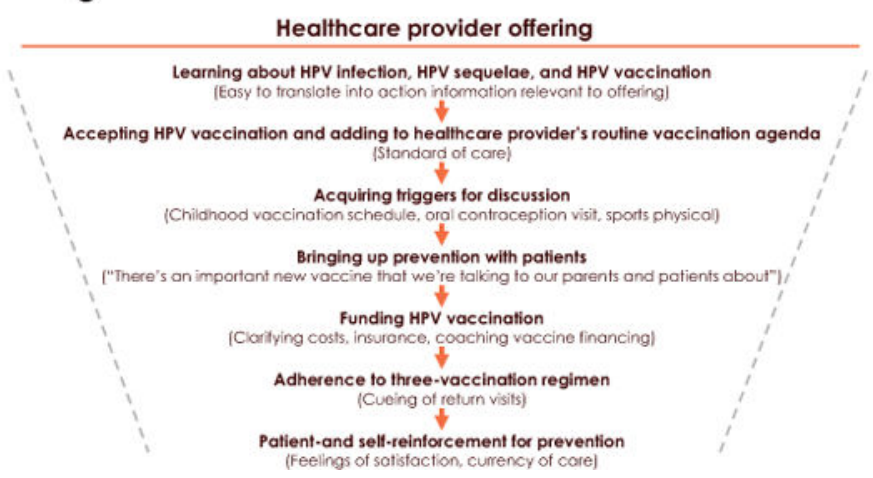

Figure 12.

The HPV vaccination behavior sequence. Seen from the perspective of (A) a parent who wishes to have their child vaccinated, (B) an adolescent or adult who wishes to be vaccinated, or (C) a health care provider who wishes to offer vaccination, there is a complex sequence of behaviors involved in vaccine series initiation and completion. Only selected vaccine uptake behavioral steps are portrayed in this figure for purposes of illustration. Reproduced with permission from Fisher WA [15]. 


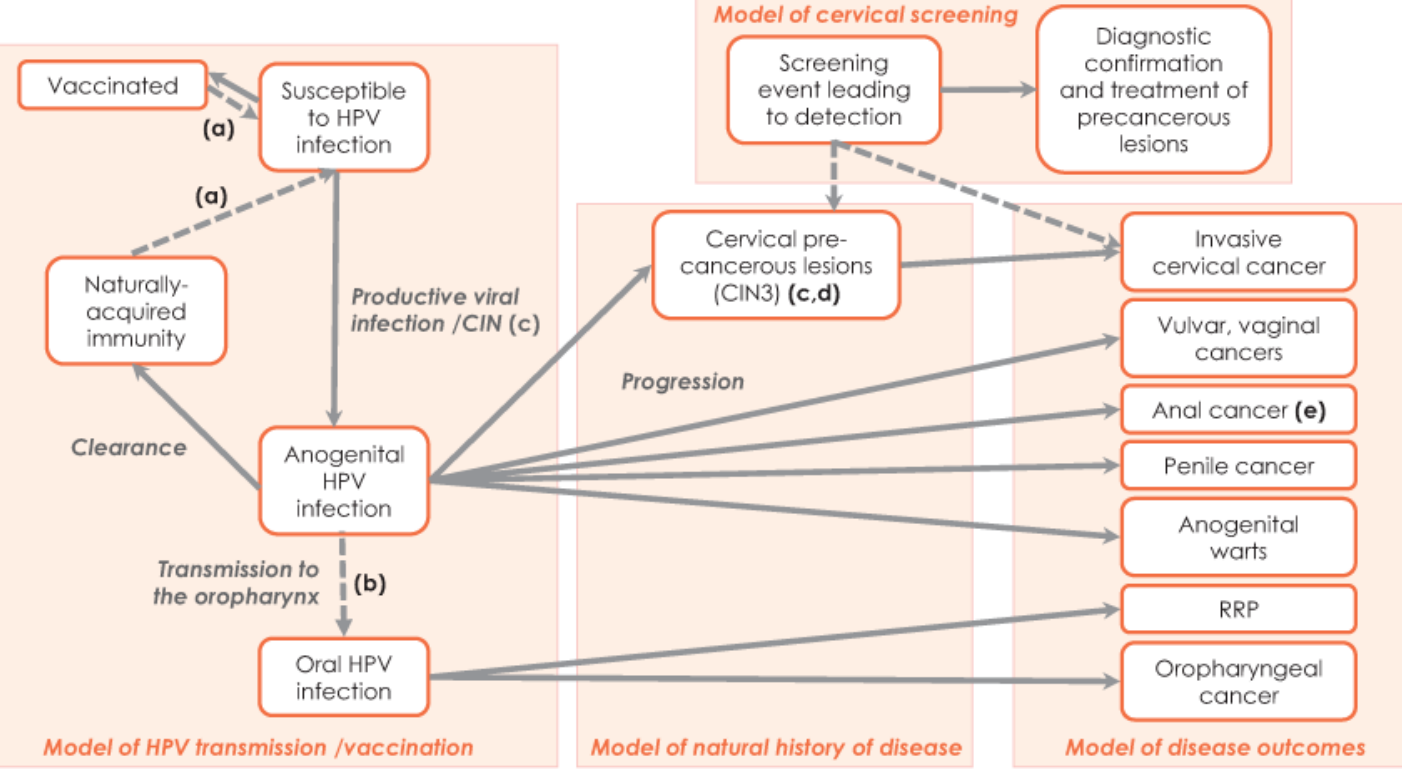

Figure 13.

Model of HPV transmission and vaccination, the natural history of disease, disease outcomes and cervical screening: Schematic diagram to inform future implementations.

(a) Type-specific vaccination and naturally-acquired immunity can be modeled. Both vaccine-induced and naturally-acquired immunity can be modeled as waning over time. If the duration of immunity to HPV infection is modeled as lifelong, individuals do not become susceptible to re-infections with the same HPV type. Although vaccine can be delivered on a population basis, only susceptible individuals can be effectively vaccinated with current generation prophylactic vaccines.

(b) Anogenital to oral HPV transmission has not generally been modeled explicitly in the evaluations conducted to date.

(c) Several models to date have used the histological CIN1-3 designation to represent natural history states, but the natural history schematic depicted here is designed to inform future evaluations by reflecting the most current biological understanding of the natural history of HPV infection of the cervix. However, this does not necessarily imply that there are no transitory states between infection and CIN3 that are relevant to modeling; rather that future models should attempt to capture the best current understanding of underlying biological processes.

(d) In practice, precancerous lesions should ideally be incorporated into models of HPV transmission since they are more likely to arise from persistent HPV infections.

(e) In the future, male vaccination may be implemented in conjunction with anal cancer screening and intermediate states (i.e., anal intraepithelial neoplasia) and this will need to be reflected in comprehensive models.

CIN: Cervical intraepithelial neoplasia; RRP: Recurrent respiratory papillomatosis.

Reproduced with permission from Canfell K et al. [16]. 


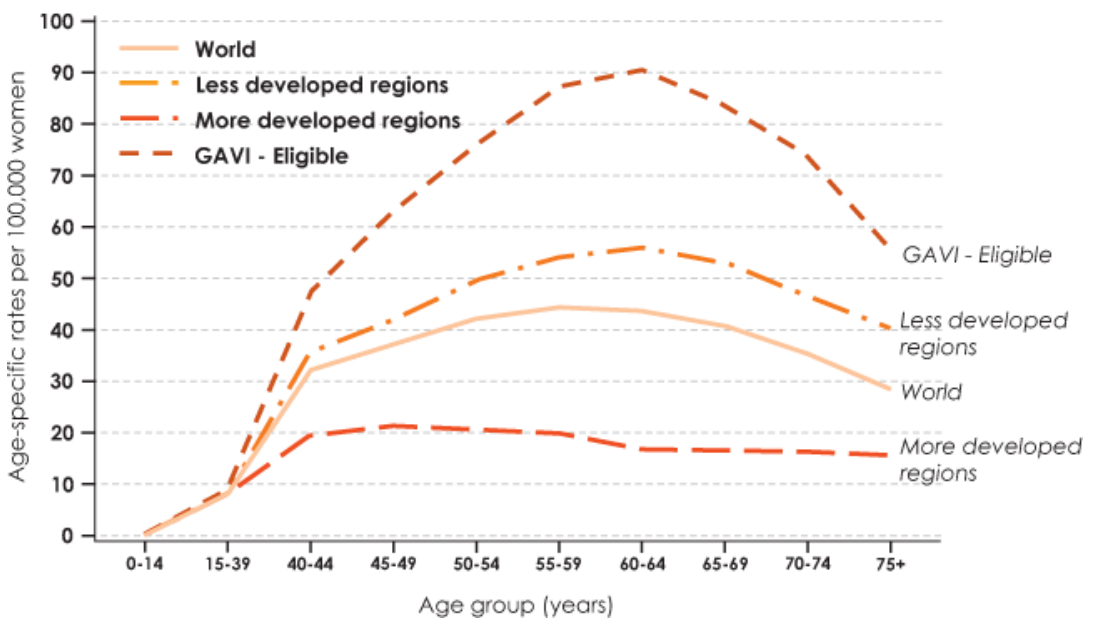

Figure 14.

Age-specific incidence rates of cervical cancer in the World, More and Less Developed regions, and GAVI-eligible countries. GAVI-eligible countries (2011): Gross national income per capita $\leq$ US $\$ 1,500$. Reproduced with permission from Kane MA et al. [20]. 


\section{Table 1}

\section{Summary of the major elements of paradigm change for prevention of HPV infection and related disease.}

Burden of disease in both genders

Vaccine efficacy in men

Vaccine accessibility

Outstanding vaccine trials
- The HPV viral etiology has been established for significant fractions of cancers of the vulva, vagina, anus in both genders and penile cancer. A significant fraction of oropharyngeal cancer in both genders is highly associated with HPV infection.

- In several industrialised countries with ongoing screening programs, data suggests that the actual number of cases of anal and oropharyngeal cancers are on the increase and may have already (or will soon) exceed that of cervical cancer.

- There has been an increased understanding of the HPV co-morbidity that occurs with HIV as well as the realization that the highest mortality from both diseases tends to occur in the same countries, often in sub-Saharan Africa.

- Data on the high rates of anal cancer in men who have sex with men, and the very high rates in HIV-positive individuals.

- $\quad$ Early data from Australia are already showing high efficacy in prevention of genital warts in immunized cohorts of females and significant but lower efficacy in unimmunized males from the same population, a significant example of the impact of herd immunity.

- Data from male efficacy studies of HPV vaccines are showing high efficacy against HPV-related anal precancerous lesions and anal cancer as well as high efficacy in preventing genital warts.

- The GAVI Alliance has included HPV in the list of vaccines to be supported financially in eligible countries opening the possibility of affordable immunization in the developing world. Vaccine prices for the quadrivalent vaccine is being offered at $5 \$ /$ dose. Many other multinational organizations (i.e., OPS/PAHO revolving fund) and national procurement offices have negotiated prices that facilitate the development of HPV vaccination public programs.

- $\quad$ Economic analyses are being redone to reflect this level of vaccine price, since the developing countries themselves will initially have to co-pay a fraction of this price.

- In the long term, adding HPV vaccine to the infant immunization schedule would lower delivery costs and make the vaccine available to almost $80 \%$ of the world's children. Even if a booster dose were needed in pre-adolescence, the strategy might be highly successful. There is a need to do the appropriate bridging studies, which can be done on a relatively small number of children.

- $\quad$ Trials on novel HPV vaccines are under way with products including additional HPV types. If successful, these vaccines are likely to dramatically modify preventive strategies, target populations and the screening protocols for vaccinated women.

Reproduced with permission from Bosch FX et al. [2]. GAVI: Global Alliance for Vaccines and Immunization: OPS/PAHO: Pan American Health Organization. 
Table 2

Results from meta-analysis showing number of women tested for HPV and HPV16, number and percent positive by cervical disease grade.

\begin{tabular}{lllll}
\hline Grade of cervical disease & $\begin{array}{l}\text { Number of women } \\
\text { tested }\end{array}$ & $\begin{array}{l}\text { Number of women } \\
\text { HPV-positive }\end{array}$ & Percentage HPV-positive & Percentage HPV16-positive $^{\boldsymbol{a}}$ \\
\hline Normal cytology & 266,611 & 33,154 & 12 & 20 \\
ASCUS & 12,983 & 6,810 & 52 & 23 \\
LSIL & 13,480 & 76 & 25 \\
HSIL & 17,805 & 6,616 & 85 & 48 \\
CIN1 & 7,743 & 8,108 & 73 & 28 \\
CIN2 & 11,043 & 4,068 & 86 & 40 \\
CIN3 & 4,754 & 10,753 & 93 & 58 \\
ICC & 11,618 & 36,374 & 89 & 63 \\
\hline
\end{tabular}

${ }^{a}$ Among HPV-positives.

ASCUS: Atypical squamous cells of undetermined significance (cytology based); CIN1: Cervical intraepithelial lesion (pathology based); CIN2/3: Cervical intraepithelial neoplasia grade 2 or 3; HSIL: High-grade squamous intraepithelial lesion (cytology based); ICC: Invasive cervical cancer (pathology based); LSIL: Low-grade squamous intraepithelial lesion (cytology based).

Reproduced with permission from Forman D et al. [3]. 


\section{Table 4}

Epidemiologic assessment of causality for HPV16 in oropharyngeal cancer.

\begin{tabular}{|c|c|}
\hline Criterion & Evidence \\
\hline Strength & $\begin{array}{l}\text { Measures of HPV } 16 \text { exposure (serologic or DNA-based) have been statistically associated in a range of } 2.3-231 \text { increased } \\
\text { risk of oropharyngeal cancer in case-control studies. }\end{array}$ \\
\hline Consistency & $\begin{array}{l}\text { HPV } 16 \text { infection has been consistently associated with increased oropharyngeal cancer risk in studies conducted across } \\
\text { different geographic locations/populations. }\end{array}$ \\
\hline Specificity & $\begin{array}{l}\text { Across head and neck cancer anatomic subsites, the association of HPV seems specific for cancers arising in the } \\
\text { oropharynx, including the base of tongue, lingual and palatine tonsil, and other parts of the oropharynx. }\end{array}$ \\
\hline Temporality & $\begin{array}{l}\text { Only one nested case-control study generated within a serum cohort study has evaluated the association of HPV with } \\
\text { prospective oropharyngeal cancer risk. HPV infection (measured by antibodies to HPV16 L1) precedes oropharyngeal } \\
\text { cancer development by up to } 15 \text { years. }\end{array}$ \\
\hline Biologic gradient & $\begin{array}{l}\text { Risk of oropharyngeal cancer increased significantly with increasing HPV16 L1 antibody titers indicating a dose-response } \\
\text { effect. }\end{array}$ \\
\hline Plausibility & $\begin{array}{l}\text { E6 and E7 proteins of HPV bind to and inactivate tumor suppressor proteins p53 and pRB, respectively, leading to } \\
\text { malignant transformation of infected cells. Studies that evaluate HPV16 E6/E7 serology found stronger associations than } \\
\text { other markers. }\end{array}$ \\
\hline Coherence & $\begin{array}{l}\text { HPV-positive oropharyngeal cancers have evidence of integrated, high copy number HPV genomes in tumor cells as well } \\
\text { as expression of E6 and E7 gene products. Consistent with HPVs being predominantly transmitted sexually, markers of } \\
\text { sexual activity, including oral sex and number of lifetime oral sex partners have also been associated with increased } \\
\text { oropharyngeal cancer risk in several studies. }\end{array}$ \\
\hline Experiment & $\begin{array}{l}\text { Downregulation of E6 and E7 oncoproteins in HPV-positive cell lines resulted in increased apoptosis and reversal of } \\
\text { malignant phenotype (as evidenced by increase in p53 and pRb levels). }\end{array}$ \\
\hline Analogy & $\begin{array}{l}\text { HPV-induced oropharyngeal carcinogenesis is analogous to HPV-induced cervical, anal, penile, vaginal, and vulvar } \\
\text { carcinogenesis. }\end{array}$ \\
\hline
\end{tabular}

Reproduced with permission from Gillison ML et al. [5]. 


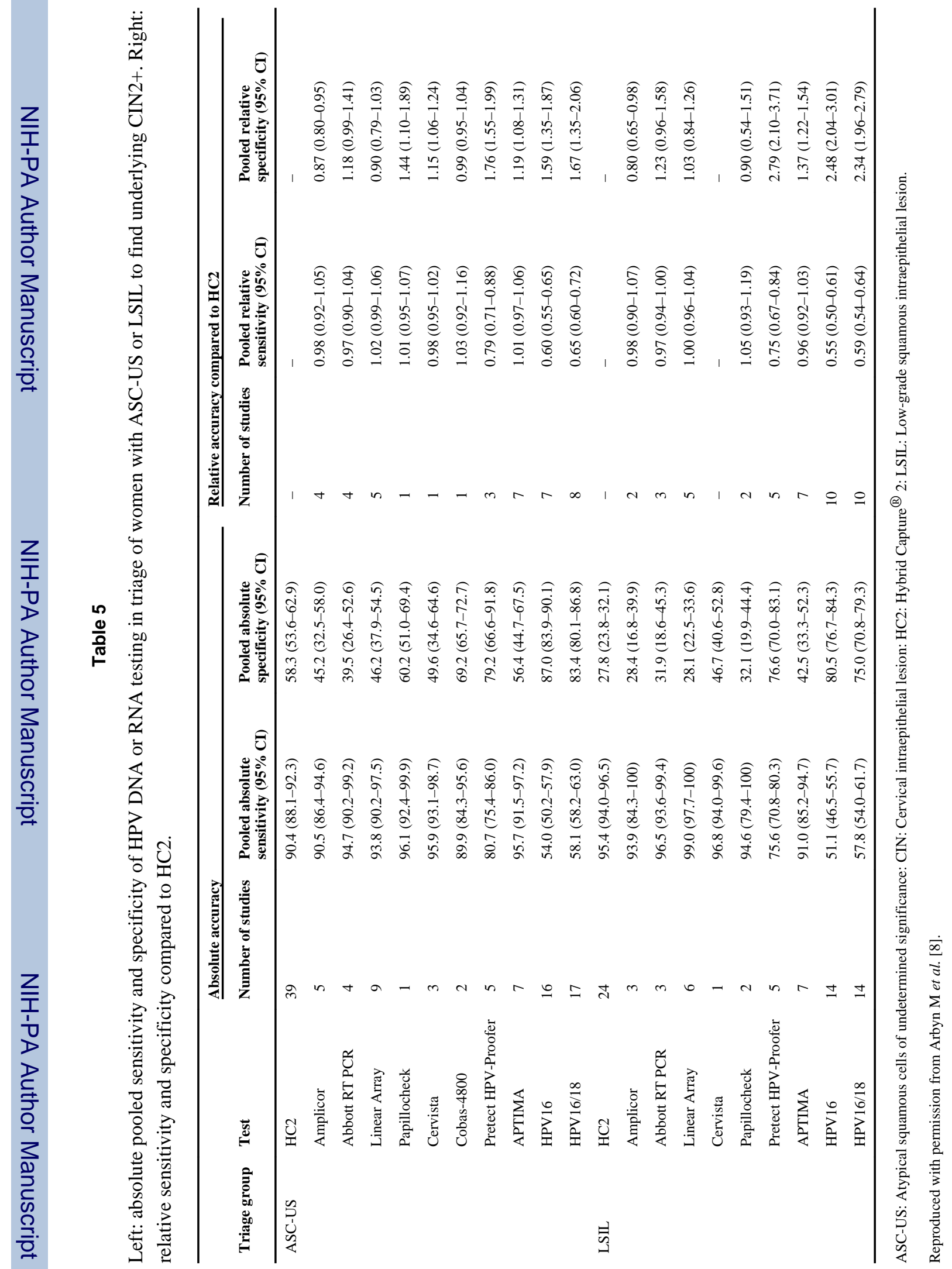




\section{Table 6}

Short list of the most widely used commercial HPV tests (status April 2012).

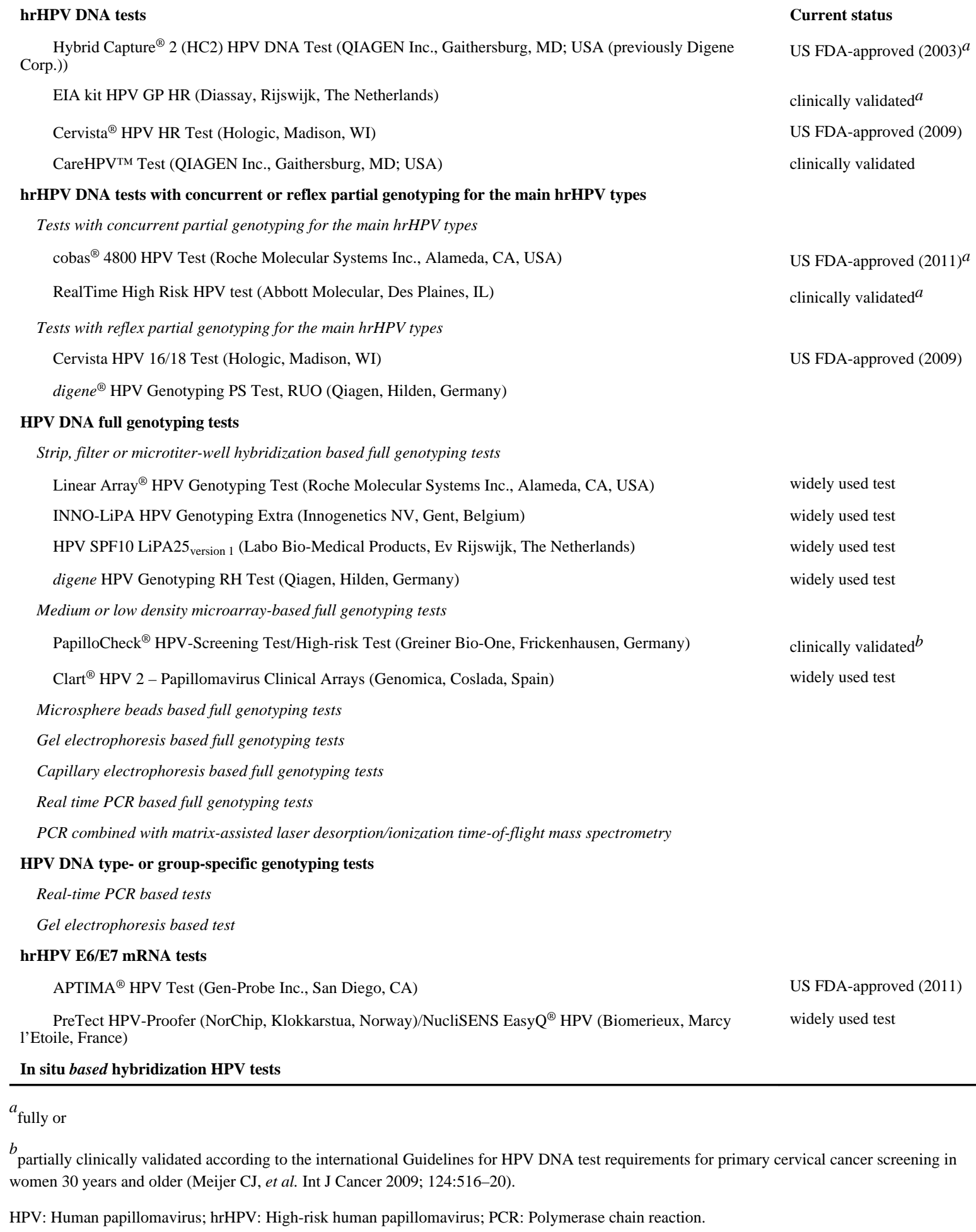

\author{
Current status \\ US FDA-approved (2003) ${ }^{a}$ \\ clinically validated ${ }^{a}$ \\ US FDA-approved (2009) \\ clinically validated
}

US FDA-approved (2011) ${ }^{a}$

clinically validated ${ }^{a}$

US FDA-approved (2009)

widely used test

widely used test

widely used test

widely used test

clinically validated $b$

widely used test

US FDA-approved (2011)

widely used test 
Reproduced with permission from Poljak M et al. [9]. 


\section{Table 7}

Technologies and procedures for cervical cancer screening reviewed in Cuzick $\mathrm{J}$ et al. [10].

\begin{tabular}{l} 
HPV typing \\
High-risk HPV \\
HPV16/18 \\
New cytologic/histologic markers \\
Biomarkers for transforming HPV-infections: p16 $6^{\mathrm{INK} 4 \mathrm{a}}$ \\
Markers for aberrant S-phase induction \\
Biomarkers for productive HPV-infections: E4 and L1 \\
Methylation of host cell genes \\
Methylation of viral genes \\
E6 and other protein markers \\
Self-sampling for HPV \\
Visual inspection techniques for cervical cancer screening \\
Visual inspection with acetic acid (VIA) \\
Visual inspection with Lugol's iodine (VILI) \\
\hline
\end{tabular}

HPV: Human papillomavirus. 
Table 8

Characteristics of HPV VLP vaccines.

\begin{tabular}{lll}
\hline & Gardasil $^{\circledR}$ & Cervarix $^{\circledR}$ \\
\hline Manufacturer & Merck & GlaxoSmithKline \\
VLP Types & $6 / 11 / 16 / 18$ & $16 / 18$ \\
Dose of L1 Protein & $20 / 40 / 40 / 20 \mu \mathrm{g}$ & $20 / 20 \mu \mathrm{g}$ \\
Producer Cells & $\begin{array}{l}\text { Saccharomyces cerevisiae (baker's yeast) } \\
\text { expressing L1 }\end{array}$ & $\begin{array}{l}\text { Trichoplusia ni (Hi 5) insect cell line infected with L1 recombinant } \\
\text { baculovirus }\end{array}$ \\
Adjuvant & $225 \mu \mathrm{g}$ aluminum hydroxyphosphate sulfate & $\begin{array}{l}\text { 500 } \mu \text { moluminum hydroxide, 50 } \mu \mathrm{g} \text { 3-O-deacylated-4'- } \\
\text { monophosphoryl lipid A }\end{array}$ \\
Injection Schedule & $0,2,6$ months & $0,1,6$ months \\
\hline
\end{tabular}

Gardasil $^{\circledR}$ (Merck \& Co., Whitehouse Station, NJ USA).

Cervarix $^{\circledR}$ (GlaxoSmithKline Biologicals, Rixensart, Belgium).

HPV: human papillomavirus; VLP: virus-like particle.

Reproduced with permission from Schiller JT et al [13]. 
Table 9

Characteristics of phase III efficacy studies in young women.

\begin{tabular}{|c|c|c|c|c|}
\hline Characteristic & FUTURE 1 & FUTURE II & PATRICIA & CVT \\
\hline Vaccine & Gardasil $^{\circledR}$ & Gardasil $^{\circledR}$ & Cervarix $^{\circledR}$ & Cervarix $^{\circledR}$ \\
\hline Funding source & Merck \& Co., Inc. & Merck \& Co., Inc. & GlaxoSmithKline & National Cancer Inst. \\
\hline No. study sites & 62 & 90 & 135 & 7 \\
\hline Countries included & 16 & 13 & 14 & 1 \\
\hline Length & 4 years & 4 years & 4 years & 4 years \\
\hline Control & $\begin{array}{l}225 \mu \mathrm{g} \text { Aluminum } \\
\text { hydroxyphosphate sulfate }\end{array}$ & $\begin{array}{l}225 \mu \mathrm{g} \text { Aluminum } \\
\text { hydroxyphosphate sulfate }\end{array}$ & Hepatitis A Vaccine & Hepatitis A Vaccine \\
\hline Age & $16-24$ & $15-26$ & $15-25$ & $18-25$ \\
\hline $\begin{array}{l}\text { Lifetime no. sexual } \\
\text { partners }\end{array}$ & $\leq 4$ & $\leq 4$ & $56^{a}$ & No restriction \\
\hline Exclusion criteria & $\begin{array}{l}\text { Pregnancy, history of } \\
\text { abnormal } \\
\text { Pap smear or genital warts }\end{array}$ & $\begin{array}{l}\text { Pregnancy, history of } \\
\text { abnormal } \\
\text { Pap smear }\end{array}$ & $\begin{array}{l}\text { Pregnancy, breastfeeding, } \\
\text { history of colposcopy, } \\
\text { autoimmume disease or } \\
\text { immunodeficiency }\end{array}$ & $\begin{array}{l}\text { Pregnancy, breastfeeding, } \\
\text { history of } \\
\text { immunosuppression, } \\
\text { hysterectomy, hepatitis A } \\
\text { vaccination }\end{array}$ \\
\hline Primary endpoints & $\begin{array}{l}\text { Incident } \\
\text { HPV6/11/16/18-associated } \\
\text { genital warts, CIN1-3, } \\
\text { VIN1-3, VaIN1-3, AIS and } \\
\text { cervical, vaginal or vulvar } \\
\text { cancer }\end{array}$ & $\begin{array}{l}\text { Incident HPV16/18 - } \\
\text { associated CIN2-3, AIS } \\
\text { or cervical cancer }\end{array}$ & $\begin{array}{l}\text { Incident HPV16/18 - } \\
\text { associated CIN2+ }\end{array}$ & $\begin{array}{l}\text { Incident } 12 \text { mo. persistent } \\
\text { HPV16/18 infection }\end{array}$ \\
\hline
\end{tabular}

$a_{\text {No limitation for Finnish subjects. }}$

AIS: Adenocarcinoma in situ; CIN: Cervical intraepithelial neoplasia; CVT: Costa Rica HPV trial; HPV: Human papillomavirus; VIN/VaIN: Vulvar/vaginal intraepithelial neoplasia.

Reproduced with permission from Schiller JT et al. [13]. 


\section{Table 10}

Protection of young women against genital disease by Gardasil ${ }^{\circledR}$ in FUTURE I and II trials.

\begin{tabular}{lll}
\hline \multicolumn{3}{l}{ A. Genital disease related to HPV6/11/16/18 } \\
\hline & \% Efficacy (95\% CI) & Rate reduction ${ }^{\boldsymbol{a}}$ \\
\hline ATP $^{b}$ & & \\
CIN2 & $100(94.7-100)$ & 0.3 \\
CIN3 & $96.8(88.1-99.6)$ & 0.3 \\
AIS & $100(30.9-100)$ & $<0.1$ \\
VIN2/3 or VaIN2/3+ & $100(82.6-100)$ & 0.1 \\
Unrestricted susceptible & & \\
CIN2 & $100(91.9-100)$ & 0.3 \\
CIN3 & $100(90.5-100)$ & 0.2 \\
AIS & $100(0-100)$ & $<0.1$ \\
VIN2/3 or VaIN2/3 & $95.4(71.5-99.9)$ & $<0.1$ \\
Genital warts & $96.4(91.4-98.9)$ & 0.8 \\
ITT & & \\
CIN2 & $54.8(40.8-65.7)$ & 0.3 \\
CIN3 & $45.1(29.8-57.3)$ & 0.3 \\
AIS & $60.0(<0-87.3)$ & $<0.1$ \\
VIN2/3 or VaIN2/3+ & $78.5(55.2-90.8)$ & $<0.1$ \\
Genital warts & $79.5(73.0-84.6)$ & 0.8 \\
&
\end{tabular}

B. Genital disease irrespective of HPV type

\begin{tabular}{lll} 
& \% Efficacy (95\% CI) & Rate reduction $^{\boldsymbol{a}}$ \\
\hline Unrestricted susceptible & & 0.3 \\
CIN2 & $42.9(20.2-59.5)$ & 0.2 \\
CIN3 & $43.0(13.0-63.2)$ & $<0.1$ \\
AIS & $100(<1-100)$ & 0.1 \\
VIN2/3 or VaIN2/3 & $77.1(47.1-91.5)$ & 0.8 \\
Genital warts & $82.8(74.3-88.8)$ & 0.2 \\
ITT & $19.3(5.7-31.0)$ & 0.2 \\
CIN2 & $16.4(0.4-30.0)$ & $<0.1$ \\
CIN3 & $62.5(<0-88.0)$ & 0.1 \\
AIS & $50.7(22.5-69.3)$ & 0.8 \\
VIN2/3 or VaIN2/3 & $62.0(53.5-69.1)$ & \\
Genital warts & &
\end{tabular}

AIS: Adenocarcinoma in situ; ATP: According to protocol; CI: Confidence interval; CIN: Cervical intraepithelial neoplasia; HPV: Human papillomavirus; ITT: Intention-to-treat; VIN/VaIN: Vulvar/vaginal intraepithelial neoplasia.

Reproduced with permission from Schiller JT et al. [13]. 


\section{Table 11}

Protection of young women against incident cervical disease by Cervarix ${ }^{\circledR}$ in the PATRICIA trial.

\begin{tabular}{|c|c|c|}
\hline \multicolumn{3}{|c|}{ A. HPV16 or HPV18-related endpoints } \\
\hline & $\%$ Efficacy $(95 \%$ CI) & Rate reduction ${ }^{a}$ \\
\hline \multicolumn{3}{|l|}{ ATP-E } \\
\hline CIN2+ & $94.9(87.7-98.4)$ & 0.38 \\
\hline CIN3+ & $91.7(66.6-99.1)$ & 0.09 \\
\hline AIS & $100(-8.6-100)$ & 0.02 \\
\hline \multicolumn{3}{|c|}{ TVC-naïve } \\
\hline CIN2+ & $99.0(94.2-100)$ & 0.47 \\
\hline CIN3+ & $100(85.5-100)$ & 0.13 \\
\hline AIS & $100(15.5-100)$ & 0.03 \\
\hline \multicolumn{3}{|l|}{ TVC } \\
\hline CIN2+ & $60.7(49.6-69.5)$ & 0.43 \\
\hline CIN3+ & $45.7(22.9-62.2)$ & 0.13 \\
\hline AIS & $70.0(-16.6-94.7)$ & 0.02 \\
\hline \multicolumn{3}{|c|}{ B. Endpoints irrespective of HPV DNA } \\
\hline & $\%$ Efficacy $(95 \%$ CI) & Rate reduction ${ }^{a}$ \\
\hline \multicolumn{3}{|c|}{ TVC-naïve } \\
\hline CIN2+ & $64.9(52.7-74.2)$ & 0.54 \\
\hline CIN3+ & $93.2(78.9-98.7)$ & 0.20 \\
\hline AIS & $100(31.0-100)$ & 0.03 \\
\hline \multicolumn{3}{|l|}{ TVC } \\
\hline CIN2+ & $33.1(22.2-42.6)$ & 0.44 \\
\hline CIN3+ & $45.6(28.8-58.7)$ & 0.22 \\
\hline AIS & $76.9(16.0-95.8)$ & 0.03 \\
\hline
\end{tabular}

$a_{\text {per } 100 \text { women years. }}$

AIS: Adenocarcinoma in situ; ATP-E: According to protocol for efficacy; CI: Confidence interval; CIN: Cervical intraepithelial neoplasia; HPV: Human papillomavirus; TVC: Total vaccine cohort.

Reproduced with permission from Schiller JT et al. [13]. 


\section{Table 12}

Cross-type protection against 6-month persistent infection.

\begin{tabular}{llll}
\hline & Efficacy $(95 \%$ CI) & & \\
Trial: & FUTURE I/II & PATRICIA & CVT \\
Vaccine: & Gardasi $^{\circledR}$ & Cervarix $^{\circledR}$ & Cervarix \\
Cohort: & ITT-Naïve $^{\circledR}$ & TVC-Naïve & ATP \\
Mean Follow-up: & $\mathbf{3 . 6}$ yrs & 3.3 yrs & 4 yrs \\
\hline HPV31 & $46.2(15.3-66.4)$ & $77.1(67.2-84.4)$ & $64.7(42.6-78.9)$ \\
HPV33 & $28.7(-45.1-65.8)$ & $43.1(19.3-60.2)$ & $32.1(-41.1-68.2)$ \\
HPV35 & $17.8(-77.1-62.5)$ & $-21.8(-102.5-26.2)$ & $25.0(-40.6-60.6)$ \\
HPV52 & $18.4(-20.6-45.0)$ & $18.9(3.2-32.2)$ & $19.6(-8.1-40.4)$ \\
HPV58 & $5.5(-54.3-42.2)$ & $-6.2(-44.0-21.6)$ & $2.8(-48.0-36.2)$ \\
Non-Vaccine A9 & $21.9(0.6-38.8)$ & $27.6(17.6-36.5)$ & NR \\
HPV39 & NR & $20.9(-2.3-39.9)$ & $-30.8(-109.2-17.6)$ \\
HPV45 & $7.8(-67.0-49.3)$ & $79.0(61.3-89.4)$ & $73.0(45.3-87.8)$ \\
HPV59 & $18.7(-22.8-46.4)$ & $-3.9(-61.7-33.1)$ & $-30.3(-130.3-25.6)$ \\
HPV68 & NR & $8.9(-18.8-30.1)$ & NR \\
Non-vaccine A7 & $14.8(-19.9-39.6)^{a}$ & $22.3(8.4-34.2)$ & NR \\
HPV51 & NR & $25.5(12.0-37.0)$ & $-56.1(-114.3-14.2)$ \\
HPV56 & NR & $1.4(-24.8-22.0)$ & $25.8(-12.7-51.4)$ \\
HPV66 & NR & $-1.5(-29.3-20.3)$ & $1.6(-41.0-31.3)$ \\
\hline & & & \\
\hline
\end{tabular}

$a_{\text {HPV45 and } 59 \text { only. }}$

ATP: According to protocol; CVT: Costa Rica HPV Trial; CI: Confidence interval; HPV: Human papillomavirus; ITT: Intention-to-treat; NR: Not reported; TVC: Total vaccine cohort.

Reproduced with permission from Schiller JT et al. [13]. 


\section{Table 13}

Efficacy of Gardasil ${ }^{\circledR}$ against HPV6/11/16/18-related end points in mid-adult women.

\begin{tabular}{lll}
\hline End point & $\begin{array}{l}\text { ATP \% Efficacy } \\
\mathbf{( 9 5 \%} \text { CI })\end{array}$ & $\begin{array}{l}\text { ITT \% Efficacy } \\
(\mathbf{9 5 \%} \text { CI) }\end{array}$ \\
\hline Persistent infection, CIN or EGL & $88.7(78.1-94.8)$ & $47.2(33.5-58.2)$ \\
Persistent infection & $89.6(79.3-95.4)$ & $49.0(35.5-59.9)$ \\
CIN - any grade & $94.1(62.5-99.9)$ & $47.5(16.3-67.7)$ \\
CIN2/3 & $83.3(-37.6-99.6)$ & $22.4(-42.5-58.3)$ \\
EGL & $100(30.8-100)$ & $8.5(-126.6-63.4)$ \\
\hline
\end{tabular}

ATP: According to protocol; CI: Confidence interval; CIN: Cervical intraepithelial neoplasia; EGL: External genital lesion; HPV: Human papillomavirus; ITT: Intention-to-treat.

Reproduced with permission from Schiller JT et al. [13]. 


\section{Table 14}

Efficacy of Gardasil ${ }^{\circledR}$ in men.

\begin{tabular}{|c|c|c|}
\hline & ATP \% Efficacy (95\% CI) & ITT \% Efficacy (95\% CI) \\
\hline \multicolumn{3}{|c|}{ External genital lesions } \\
\hline Any Type ${ }^{a}$ & $83.8(61.2-94.4)$ & $60.2(40.8-73.8)$ \\
\hline HPV6.11, 16, 18 & $90.4(69.2-98.1)$ & $65.5(45.8-78.6)$ \\
\hline \multicolumn{3}{|c|}{ Persistent genital infection ${ }^{b}$} \\
\hline HPV6,11, 16, 18 & $85.6(73.4-92.9)^{c}$ & $47.8(36.0-57.6)$ \\
\hline HPV6 & NR & $44.7(24.1-60.1)$ \\
\hline HPV11 & NR & $59.4(25.7-78.8)$ \\
\hline HPV16 & 78.7 (55.5-90.9) & $46.9(28.6-60.8)$ \\
\hline HPV18 & $96.0(75.6-99.9)$ & $56.0(28.2-73.7)$ \\
\hline \multicolumn{3}{|l|}{ AIN-any grade } \\
\hline Any Type ${ }^{a}$ & $54.9(8.4-79.1)$ & $25.7(-1.1-45.6)$ \\
\hline HPV6, 11, 16, 18 & $77.5(39.6-93.3)$ & $50.3(25.7-67.2)$ \\
\hline AIN2/3 & $74.9(8.8-95.4)$ & $54.2(18.0-75.3)$ \\
\hline \multicolumn{3}{|c|}{ Persistent anal infection } \\
\hline HPV6, 11, 16, 18 & $94.9(80.4-99.4)$ & $59.4(43.0-71.4)$ \\
\hline HPV6 & $92.1(47.2-99.8)$ & $62.5(37.5-78.2)$ \\
\hline HPV11 & $100(-15.5-100)$ & $53.7(7.5-78.0)$ \\
\hline HPV16 & $93.8(60.0-99.9)$ & $54.0(23.9-72.9)$ \\
\hline HPV18 & $100(51.5-100)$ & $73.6(37.5-90.3)$ \\
\hline
\end{tabular}

${ }^{a}$ Seronegative to HPV6, 11, 16, 18 and DNA negative to 6, 11, 16, 18, 31, 33, 35, 39, 45, 51, 56, 58, and 59 at enrollment.

${ }^{b}$ Persistence defined as detection of the same type on consecutive visits at least $6(+/-1)$ months apart.

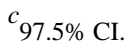

AIN: Anal intraepithelial neoplasia; ATP: According to protocol; CI: Confidence interval; HPV: Human papillomavirus; ITT: Intention-to-treat; NR: Not reported.

Reproduced with permission from Schiller JT et al. [13]. 
Table 16

Key findings from clinical trials of HPV VLP vaccines.

\begin{tabular}{|c|c|c|c|}
\hline Study group & Outcome & Gardasil $^{\circledR}$ & Cervarix $^{\circledR}$ \\
\hline \multirow[t]{10}{*}{ Young women } & Infection efficacy & Proven & Proven \\
\hline & CIN2+ efficacy & Proven & Proven \\
\hline & CIN3 efficacy & Proven & Proven \\
\hline & VIN/VaIN 2/3 efficacy & Proven & Proven $^{a}$ \\
\hline & Genital warts efficacy & Proven & Not a target \\
\hline & Anal infection efficacy & Not proven & Proven \\
\hline & Partial cross-protection infection & Proven & Proven \\
\hline & Partial cross-protection CIN2+ & Proven & Proven \\
\hline & Therapeutic efficacy & None & None \\
\hline & Safety & No concerns & No concerns \\
\hline \multirow[t]{4}{*}{ Mid-adult women } & Infection efficacy & Proven & Proven $^{a}$ \\
\hline & CIN2+ efficacy & Proven & Not proven \\
\hline & Immunogenicity & Proven & Proven \\
\hline & Safety & No concerns & No concerns \\
\hline \multirow[t]{5}{*}{ Young men } & Infection efficacy & Proven & Not proven \\
\hline & Genital wart efficacy & Proven & Not a target \\
\hline & Anal infection & Proven & Not proven \\
\hline & AIN2+ efficacy & Proven & Not proven \\
\hline & Safety & No concerns & No concerns \\
\hline \multirow[t]{4}{*}{ Children } & Infection efficacy & Not proven & Not proven \\
\hline & Disease efficacy & Not proven & Not proven \\
\hline & Immunogenicity & Proven & Proven \\
\hline & Safety & No concerns & No concerns \\
\hline
\end{tabular}

${ }^{a}$ Meeting abstract but not yet published.

CIN: Cervical intraepithelial neoplasia; HPV: Human papillomavirus; VIN/VaIN: Vulvar/vaginal intraepithelial neoplasia; VLP: Virus-like particle.

Reproduced with permission from Schiller JT et al. [13]. 


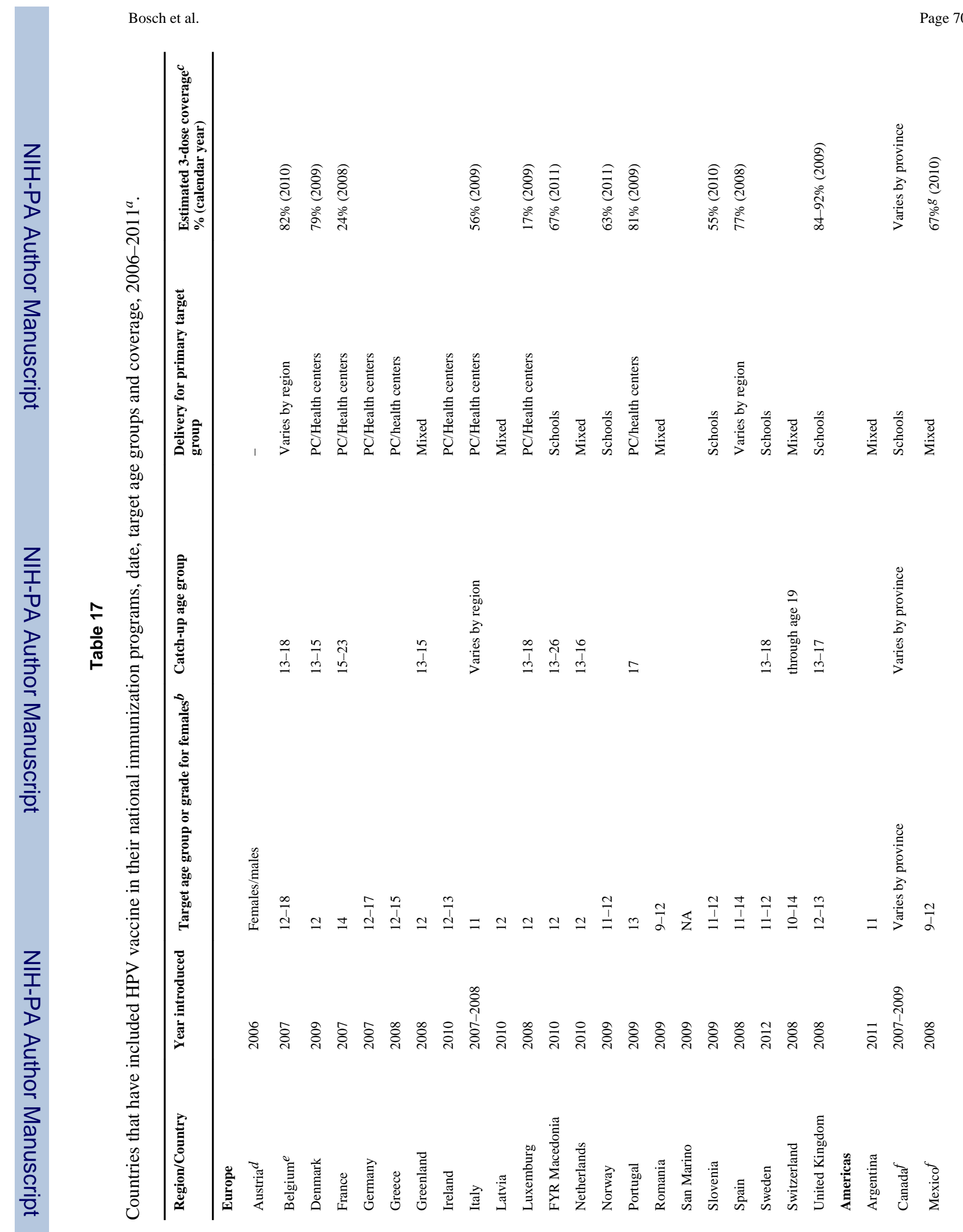

Vaccine. Author manuscript; available in PMC 2014 November 22. 
Bosch et al.

Page 71

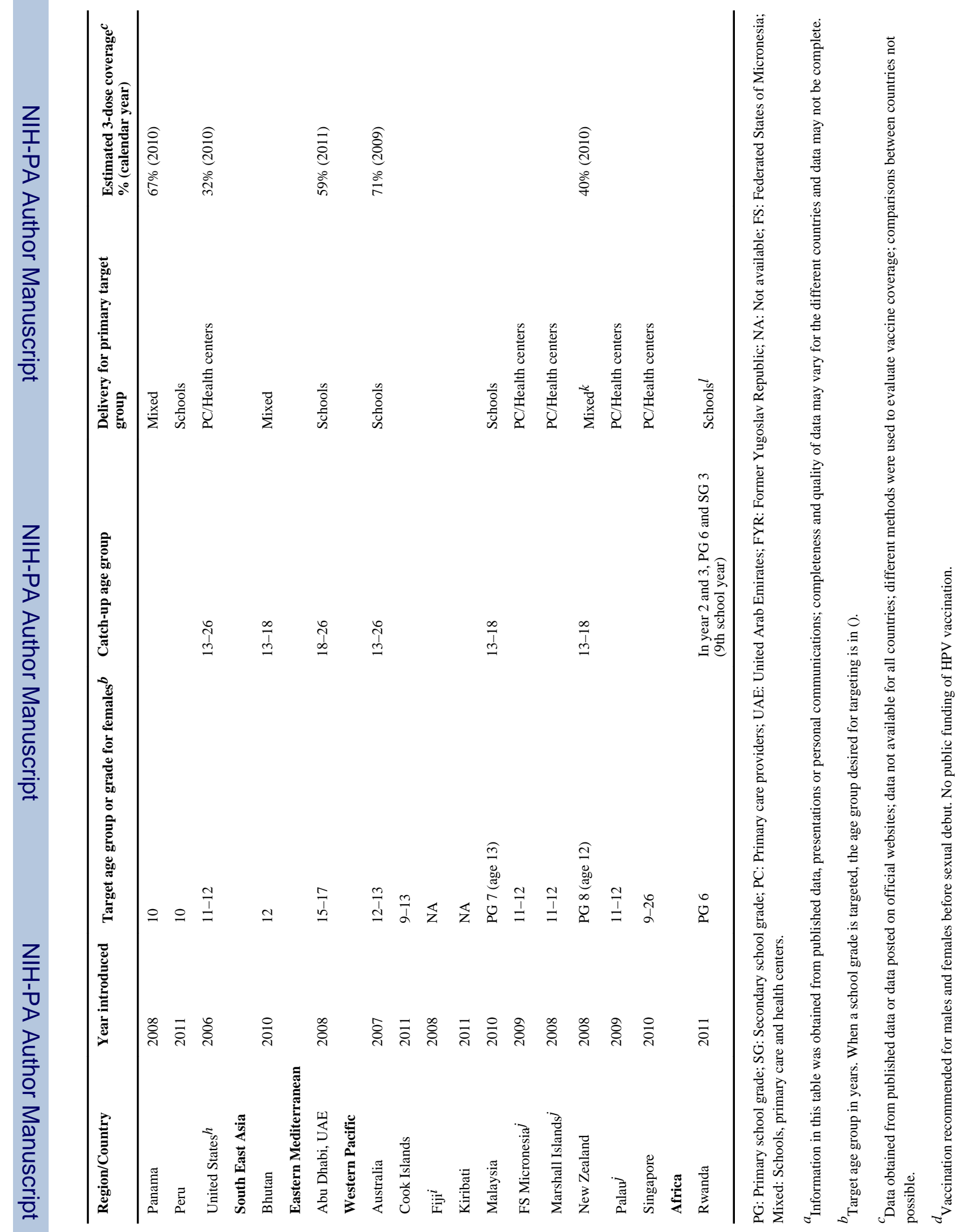




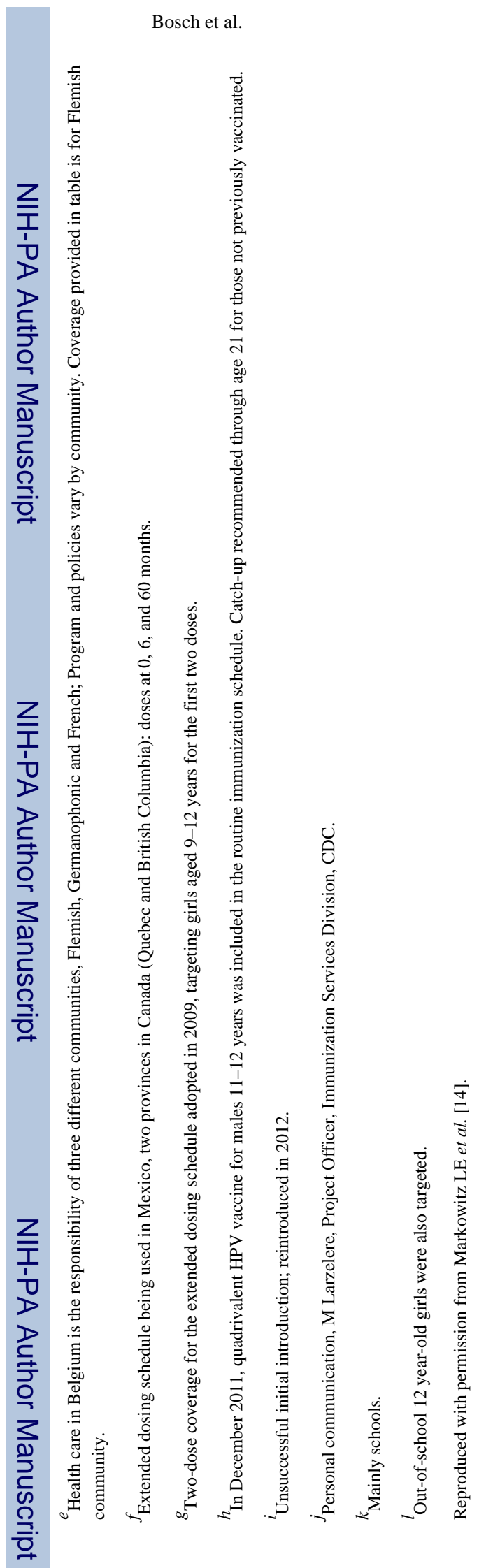

Vaccine. Author manuscript; available in PMC 2014 November 22. 


\section{Table 18}

Demonstration projects and strategy refinements after evaluation of pilot programs in three low-resource countries. $^{a}$

\begin{tabular}{|c|c|c|c|}
\hline Country & $\begin{array}{l}\text { Pilot strategy }(n=\text { girls } \\
\text { eligible for } \\
\text { vaccination) }\end{array}$ & Key evaluation findings & Strategy refinements \\
\hline \multirow[t]{2}{*}{ Peru } & Schools $(n=8,092)$ & $\begin{array}{l}\text { Easy to access girls in densely populated areas but very } \\
\text { inefficient for small schools in remote areas. }\end{array}$ & $\begin{array}{l}\text { Combine HPV vaccine with routine EPI } \\
\text { nurse visits to remote areas. }\end{array}$ \\
\hline & $\begin{array}{l}\text { Health Centers }(n= \\
8,060)\end{array}$ & $\begin{array}{l}\text { Uptake of first dose was slow and required additional } \\
\text { reminders in community. }\end{array}$ & $\begin{array}{l}\text { Use school-based vaccination to reach the } \\
\text { largest percentage of eligible girls more } \\
\text { easily. }\end{array}$ \\
\hline \multirow[t]{2}{*}{ Uganda } & Schools $(n=6,294)$ & $\begin{array}{l}\text { Schools were a convenient location to find large number } \\
\text { of girls, but special outreach visits were costly to } \\
\text { organize. }\end{array}$ & $\begin{array}{l}\text { Use schools as vaccination location but } \\
\text { integrate with another community health } \\
\text { program. }\end{array}$ \\
\hline & $\begin{array}{l}\text { Child Plus Days }(n= \\
4,183)\end{array}$ & $\begin{array}{l}\text { Program was more efficient but selection of girls by age } \\
\text { resulted in lower coverage, as age verification was } \\
\text { difficult. }\end{array}$ & Select girls by grade rather than age. \\
\hline \multirow[t]{2}{*}{ Vietnam } & Schools $(n=4,302)$ & $\begin{array}{l}\text { Parent meetings at schools facilitated information and } \\
\text { education activities. Vaccination days scheduled outside } \\
\text { normal school year challenging. }\end{array}$ & $\begin{array}{l}\text { Set vaccination schedule during the school } \\
\text { year if school-based strategy used. }\end{array}$ \\
\hline & $\begin{array}{l}\text { Health Centers }(n= \\
2,712)\end{array}$ & $\begin{array}{l}\text { Confirmed that health centers used for HPV vaccine } \\
\text { could achieve good coverage. }\end{array}$ & $\begin{array}{l}\text { Consider health center-based approach for } \\
\text { future national scale up. }\end{array}$ \\
\hline
\end{tabular}




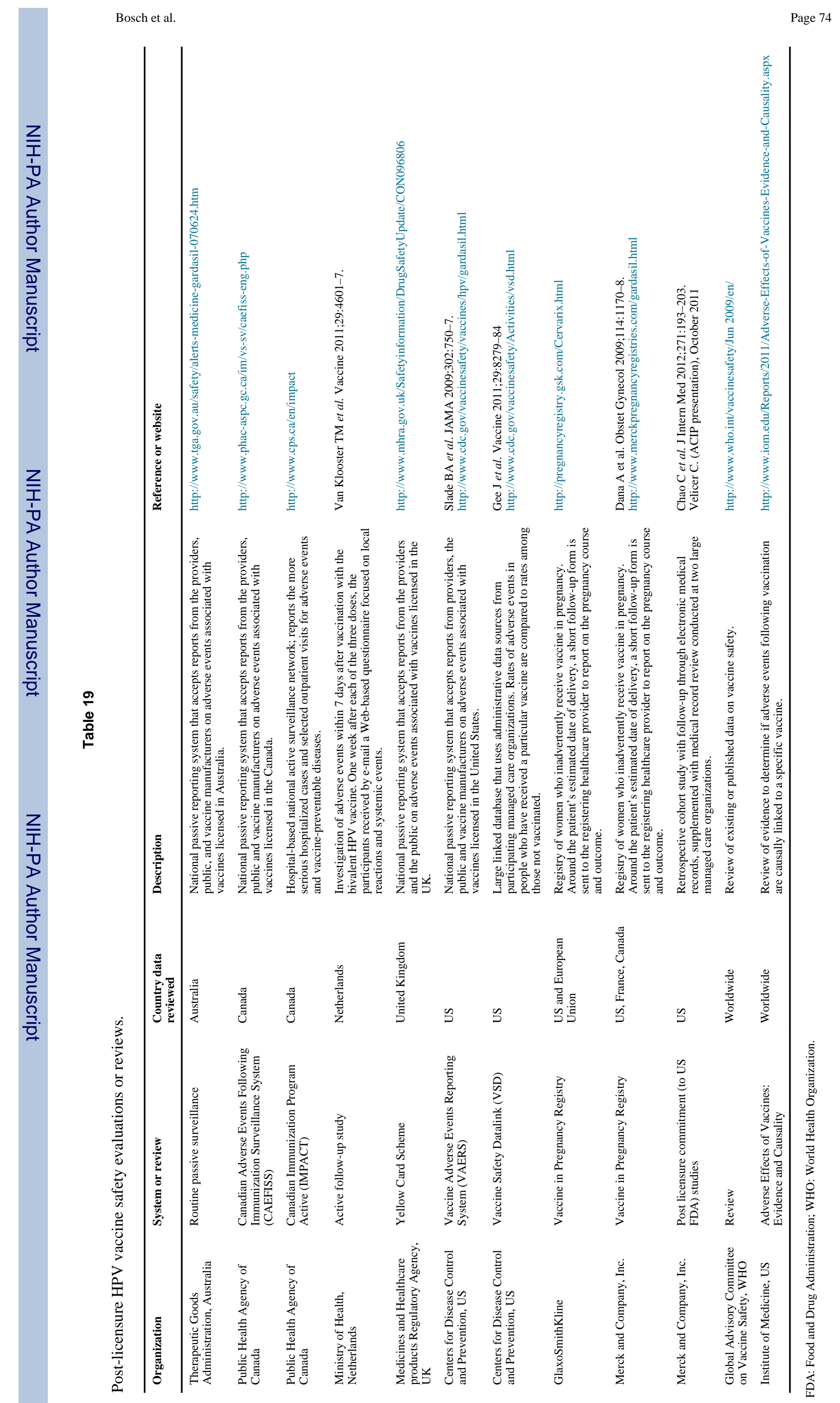




\section{Table 20}

Gaps in knowledge and pertinent research issues and hypotheses regarding the role of HPV vaccination as a primary prevention strategy.

\begin{tabular}{|c|c|}
\hline Key questions & Research issues and ancillary hypotheses \\
\hline $\begin{array}{l}\text { What is the extent of cross-type } \\
\text { protection by the existing L1 VLP-based } \\
\text { vaccines: are benefits to be expected at } \\
\text { the population level? }\end{array}$ & $\begin{array}{l}\text { Cross-protection for types that are phylogenetically close to the vaccine types seems real but } \\
\text { limited in efficacy and duration of protection. Differences between the vaccines, if real, could } \\
\text { suggest adjuvant effects or be a result of how VLPs are produced. }\end{array}$ \\
\hline $\begin{array}{l}\text { Can correlates of immune protection be } \\
\text { identified? }\end{array}$ & $\begin{array}{l}\text { Serologic antibody titers post-vaccination or other immune markers do not predict protection at an } \\
\text { individual level. Long-term follow-up of vaccinated populations may shed light on determinants of } \\
\text { protection. Research is needed on different definitions of viral or lesion outcomes. }\end{array}$ \\
\hline $\begin{array}{l}\text { How many vaccine doses are needed? } \\
\text { Could fewer doses provide protection? } \\
\text { Could different injection intervals } \\
\text { achieve equal protection? }\end{array}$ & $\begin{array}{l}\text { Regulatory RCTs were designed to address the efficacy of three-dose regimens. Simplified } \\
\text { regimens with fewer doses or different scheduling could enhance coverage and decrease costs of } \\
\text { deploying vaccination. Can fewer doses elicit long-lasting protection? }\end{array}$ \\
\hline $\begin{array}{l}\text { Anamnestic response by sexual exposure } \\
\text { post-HPV vaccination: is it expected? }\end{array}$ & $\begin{array}{l}\text { Natural boosting of the immune response post-vaccination via sexual exposure to HPV infection } \\
\text { could be examined in surveillance studies augmented by behavioral questionnaires. Is antigenic } \\
\text { exposure high enough to heighten serological titers? Would response times be sufficient to prevent } \\
\text { infection? }\end{array}$ \\
\hline $\begin{array}{l}\text { Is protection expected to be pan- } \\
\text { mucosal? }\end{array}$ & $\begin{array}{l}\text { Plausibly, vaccination exerts a prophylactic effect in all mucosal sites that serve as port of entry for } \\
\text { HPV infections. However, there is scant data to document protection against new infections or } \\
\text { lesions in non-cervical sites. }\end{array}$ \\
\hline $\begin{array}{l}\text { Does vaccination prevent recurrent } \\
\text { infection in the same, adjacent, or distant } \\
\text { mucosal sites? }\end{array}$ & $\begin{array}{l}\text { Vaccination will not clear existing infections but may have a protective effect in adjacent areas, } \\
\text { thus potentially having a benefit in preventing multi-focal infections and recurrent lesions in the } \\
\text { cervix, vagina, and oral sites. More research is needed on mucosal immunity. }\end{array}$ \\
\hline $\begin{array}{l}\text { Is type replacement to be expected post- } \\
\text { vaccination? Can vaccination be } \\
\text { detrimental for the natural history of } \\
\text { non-vaccine-target HPV types? What are } \\
\text { the methodologic caveats in } \\
\text { investigating this possibility? }\end{array}$ & $\begin{array}{l}\text { HPVs are highly stable DNA viruses; thus, selective pressures from vaccination may not elicit the } \\
\text { emergence of new types but may vacate existing ecological niches currently taken by HPVs16/18. } \\
\text { Long-term follow-up of vaccinated populations will provide answers but analyses of existing } \\
\text { cohorts can provide valuable insights as to whether or not some types are presently out-competed } \\
\text { by HPVs } 16 \text { and } 18 \text { and could thus increase in prevalence later. }\end{array}$ \\
\hline Should boys be vaccinated? & $\begin{array}{l}\text { As one of the currently most pressing questions, it remains one of affordability for most countries. } \\
\text { The benefits are the protection against HPV-associated diseases in men and the enhanced herd } \\
\text { immunity with consequent reduction in HPV transmission in populations (ultimately benefiting } \\
\text { both genders). Can countries attain sufficiently high male vaccination coverage rates? }\end{array}$ \\
\hline
\end{tabular}

RCTs: Randomized, controlled trials; VLP: Virus-like particle.

Reproduced with permission from Franco EL et al. [18]. 


\section{Table 21}

Gaps in knowledge and pertinent research issues and hypotheses regarding the role of screening technologies in secondary cervical cancer prevention.

\begin{tabular}{|c|c|}
\hline Key questions & Research and implementation issues \\
\hline $\begin{array}{l}\text { What answers are still needed from the } \\
\text { studies of HPV testing in screening? }\end{array}$ & $\begin{array}{l}\text { Is there sufficient buy-in for wide-scale implementation in high-resource settings? Can HPV DNA } \\
\text { or RNA testing be implemented cost-effectively in middle- and low-resource settings? }\end{array}$ \\
\hline $\begin{array}{l}\text { Cotesting versus serial testing: what is the } \\
\text { best option for high-resource settings? }\end{array}$ & $\begin{array}{l}\text { Few countries have formally included cotesting (parallel use of HPV plus Pap cytology) in } \\
\text { practice guidelines. Can serial testing (HPV followed by Pap triage of HPV positives) attain the } \\
\text { same level of safety for guidelines? }\end{array}$ \\
\hline $\begin{array}{l}\text { If HPV testing is adopted for women ages } \\
30 \text { and older, what screening options } \\
\text { should be recommended for younger } \\
\text { women? }\end{array}$ & $\begin{array}{l}\text { The technology "neglected" age range of } 21-29 \text { years continues to rely on cytology. What types } \\
\text { of evidence will be required for increasing the age of screening initiation? Could a compromise } \\
\text { solution exist via a single policy of serial testing (HPV followed by Pap triage) beginning at age } \\
25 ?\end{array}$ \\
\hline $\begin{array}{l}\text { Is VIA a solution for low-resource } \\
\text { settings, either alone or as triage for low- } \\
\text { cost HPV primary screening? }\end{array}$ & $\begin{array}{l}\text { VIA is not as accurate as HPV testing but is easier to deploy. Is it a method that should only be } \\
\text { combined with screen-and-treat strategies? What is the value of VIA for the triage of HPV- } \\
\text { positive women to improve the effectiveness of screen-and-treat strategies? }\end{array}$ \\
\hline $\begin{array}{l}\text { Is self-sampling a solution to expand the } \\
\text { coverage and bring equity to screening? }\end{array}$ & $\begin{array}{l}\text { HPV testing of self-collected samples could permit reaching remote areas, urban women who are } \\
\text { missed by invitations to screen, and women who refuse provider-assisted sampling. Is the balance } \\
\text { between lower accuracy and higher coverage acceptable? }\end{array}$ \\
\hline $\begin{array}{l}\text { Algorithm management versus risk } \\
\text { stratification: what is most suitable for } \\
\text { guidelines? }\end{array}$ & $\begin{array}{l}\text { Can healthcare providers learn and apply risk stratification via multiple biomarker testing as part } \\
\text { of practice guidelines? Does it confer a more personalized level for screening and management? } \\
\text { Is it cost-effective? }\end{array}$ \\
\hline $\begin{array}{l}\text { What is the role of HPV viral load as a } \\
\text { clinical tool? }\end{array}$ & $\begin{array}{l}\text { Should HPV testing be based on higher thresholds of viral load for improved specificity? Is the } \\
\text { greater complexity of quantitative HPV assays worth the extra cost to be borne in screening? }\end{array}$ \\
\hline $\begin{array}{l}\text { Is there a role for genotyping in screening } \\
\text { or triage? }\end{array}$ & $\begin{array}{l}\text { Genotyping for HPVs } 16,18 \text {, and other priority hrHPVs improves the positive predictive value of } \\
\text { screening and permits more rational colposcopy referral. Can genotyping become affordable in } \\
\text { the near future to be implemented in screening, triage, and surveillance? }\end{array}$ \\
\hline $\begin{array}{l}\text { What is the role of cytology-based } \\
\text { staining for prognostic markers of lesion } \\
\text { progression? }\end{array}$ & $\begin{array}{l}\text { Is the accrued accuracy of enhancements in cytology based on the identification of these markers } \\
\text { worth the added cost to cytologic triage of HPV positive women? Can it compete with genotyping } \\
\text { as a cost-effective strategy? }\end{array}$ \\
\hline $\begin{array}{l}\text { How should we educate healthcare } \\
\text { providers and patients concerning HPV } \\
\text { testing results? }\end{array}$ & $\begin{array}{l}\text { Gradual introduction of HPV testing leads to patient anxiety and confusion related to the diversity } \\
\text { of guidelines. The change from an oncologic to an STI-detection paradigm in cervical cancer } \\
\text { screening requires research on sound educational approaches to demystify the implications of } \\
\text { HPV positive results. }\end{array}$ \\
\hline $\begin{array}{l}\text { What will be the impact of HPV } \\
\text { vaccination on screening performance? }\end{array}$ & $\begin{array}{l}\text { As vaccinated young women reach the age of screening, a gradual decrease in lesion prevalence } \\
\text { will adversely impact test performance. Which tests will be less likely to be affected? Can } \\
\text { guidelines be safely relaxed in HPV-vaccinated populations? Can HPV-based screening be } \\
\text { integrated with HPV vaccination strategies for shared resources and improved surveillance? }\end{array}$ \\
\hline
\end{tabular}

hrHPV: High-risk human papillomavirus: STI: Sexually-transmitted infection: VIA: Visual inspection with acetic acid.

Reproduced with permission from Franco EL et al. [18]. 
Table 22

Tips for taking a step forward in HPV prevention.

\section{Cervical cancer screening}

If no program exists, plan a VIA pilot project

If a program exists, plan expansion of coverage or tests, including HPV DNA base tests

Develop a communication program for women in the targeted populations

\section{Prophylactic vaccines}

If no program exists, plan a pilot project

Develop strategies with the help of local health care settings leaders for delivery of care or immunizations to the target population

\section{Research and evaluation}

If no program exists, plan a survey on knowledge, attitudes and behaviors of managers and health care professionals

Evaluate the quality of national cancer registry

Develop a quality control program for delivery of care or lab results

Health promotion and education

If no program exists, plan a communication strategy about HPV and its prevention for managers, health care professionals and target population Involve community leaders in the development of strategies

Reproduced with permission from Steben M et al. [19]. 
Table 23

Important factors in the policy process for HPV vaccine introduction in Low-Resource Settings.

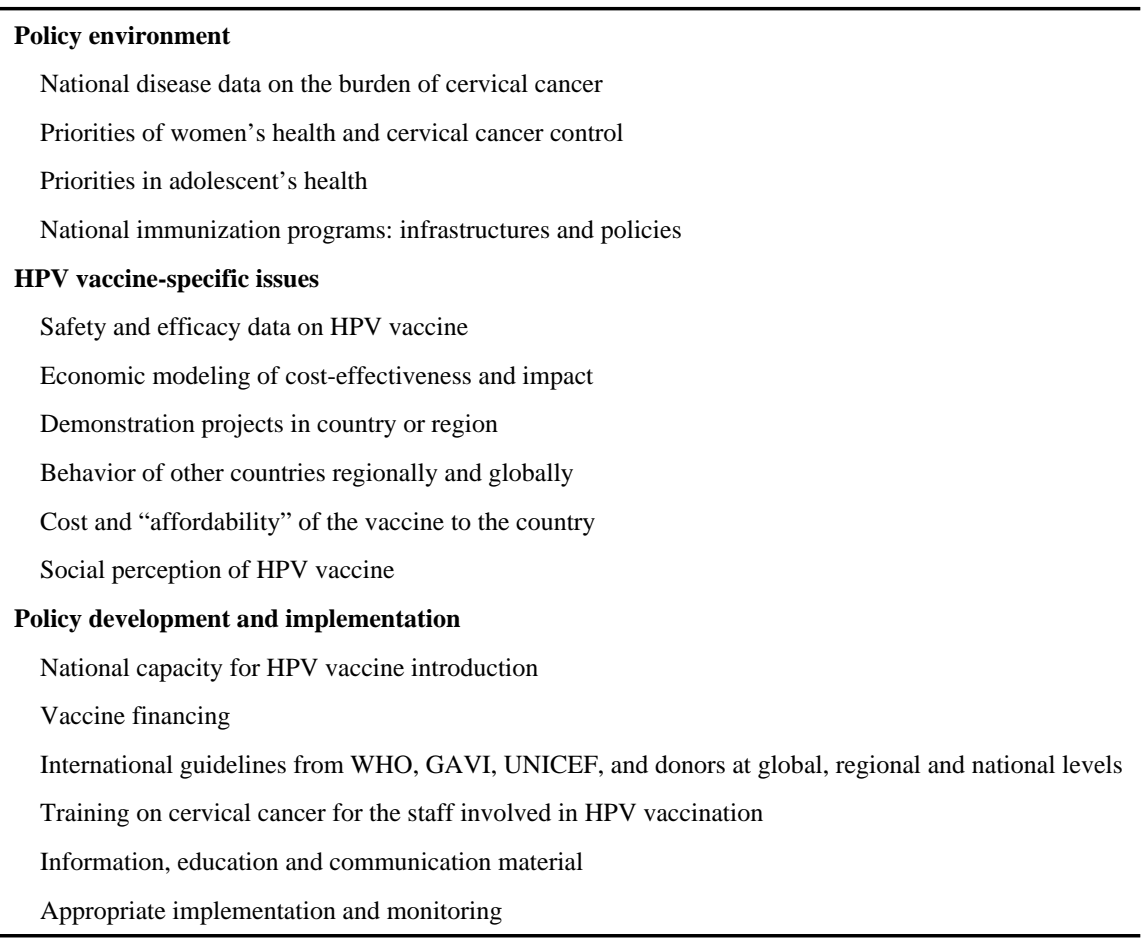

UNICEF: United Nations Children's Fund: WHO: World Health Organization. Reproduced with permission from Kane MA et al. [20]. 